\title{
The Dynamics of Bertrand Price Competition with Cost-Reducing Investments ${ }^{\dagger}$
}

\author{
Fedor Iskhakov* \\ Australian National University \\ John Rust" \\ Georgetown University \\ Bertel Schjerning \& \\ University of Copenhagen
}

August, 2017

\begin{abstract}
We extend the classic Bertrand duopoly model of price competition to a dynamic setting where competing duopolists invest in a stochastically improving production technology to "leapfrog" their rival and attain temporary low cost leadership. We find a huge multiplicity of Markov perfect equilibria (MPE) and show that when firms move simultaneously the set of all MPE payoffs is a triangle that includes monopoly payoffs and a symmetric zero mixed strategy payoff. When firms move asynchronously, the set of MPE payoffs is strictly within this triangle, but there still is a vast multiplicity of MPE, most of which involve leapfrogging.
\end{abstract}

Keywords: duopoly, Bertrand-Nash price competition, Bertrand investment paradox, leapfrogging, costreducing investments, technological improvement, dynamic models of competition, Markov-perfect equilibrium, tacit collusion, price wars, coordination and anti-coordination games, strategic preemption

JEL classification: D92, L11, L13

$\dagger$ We acknowledge helpful comments from Michael Baye, Jeffrey Campbell, Dan Cao, Joseph E. Harrington, Jr., Dan Kovenock, Roger Lagunoff, Stephen Morris, Michael Riordan, Jean-Marc Robin, David Salant, Karl Schmedders, Che-Lin Su, and other participants at seminars at Columbia, University of Copenhagen, CREST, Georgetown, Princeton, the Chicago Booth School of Business, University of New South Wales, the 3rd CAPCP conference at Pennsylvania State University, the 2012 conferences of the Society of Economic Dynamics, the Society for Computational Economics, the North American Summer Meetings of the Econometric Society, the 4th World Congress of the Game Theory Society, the Initiative for Computational Economics at Chicago and Zurich (ICE 2012 and ZICE 2013 to 2015), and the NSF/NBER CEME Conference on "The Econometrics of Dynamic Games." The authors would like to acknowledge the funding received from the Danish Council for Independent Research and Innovation Fund Denmark. Fedor Iskhakov gratefully acknowledges the support from the Australian Research Council projects CE110001029 and FL110100247 as well as from Frisch Centre project 1307 financed by the Ministry of Labor, Norway.

* Email: fedor.iskhakov@anu.edu.au

* Correspondence address: Department of Economics, Georgetown University, Washington, DC, phone: (301) 801-0081, email: jr1393egeorgetown.edu

$\S$ Email: bertel.schjerning@econ.ku.dk 


\section{Introduction}

Despite the huge literature spawned by Bertrand (1883), our understanding of price competition in the presence of production cost uncertainty is still rudimentary. For example, even in the static Bertrand model, Routlege (2010) notes that "there is a notable gap in the research. There are no equilibrium existence results for the classical Bertrand model when there is discrete cost uncertainty." (p. 357). Less is known about Bertrand price competition in dynamic models where firms compete via cost-reducing investments, and are uncertain about their rivals' investment decisions and the timing of technological innovations that can affect future prices and costs of production.

This paper analyses a simple dynamic extension of the textbook Bertrand-Nash duopoly model of price competition where the firms can make investment decisions as well as pricing decisions. At any time $t$ a firm can decide whether to replace its current production plant with a new state of the art production facility that enables it to produce at a lower marginal cost than its rival. Thus, we assume that the state of the art technology improves stochastically and exogenously, whereas technology adoption decisions are endogenous.

The term leapfrogging describes the longer run investment competition where a higher cost firm purchases a state of the art production technology that reduces its marginal cost relative to its rival and allows it to attain, at least temporarily, a position of low cost leadership. The assumption that the state of the art technology evolves exogenously differentiates our model from earlier examples of leapfrogging in the literature by, for example, Fudenberg et. al. (1983) and Reinganum (1985). This earlier work on patent races and models of research and development focused on firms' continuous choice of $R \& D$ expenditures with the goal of producing a patent or a drastic 
innovation that could not be easily duplicated by rivals.

However, there are industries where firms do little or no R\&D themselves but can leapfrog their rivals by investing in a state of the art production technology that is developed and sold by third parties. We model this investment as a binary decision: each firm faces a decision of whether or not to incur the substantial fixed investment cost to replace their current legacy production technology with the latest technology in order to become the current low cost leader. Since all firms have equal opportunity to acquire the state of the art production technology the markets we study differ from those studied in the earlier literature on leapfrogging in the context of $R \& D$ and patent races. Though the market we study abstracts from entry and exit, it is contestable due to ease of investment. This may promote competition in ways that are similar to markets that are contestable due to ease of entry (Baumol, Panzar and Willig, 1982).

The ease by which either firm can invest in our model leads to an issue we call the "Bertrand investment paradox". If both firms invest at the same time, then Bertrand price competition drives ex post profits to zero. If the firms expect this, the ex ante return on their investments will be negative, so it is possible that no firm would have an incentive to undertake cost-reducing investments. But if no firm invests, it may make sense for at least one firm to invest. Thus, the investment problem has the structure of an anti-coordination game.

Riordan and Salant $(\mathrm{RS}, 1994)$ showed how the Bertrand investment paradox can be resolved. They analyzed a model of Bertrand price competition between duopolists who can both invest to acquire a deterministically improving state of the art technology to try to gain a temporary cost advantage over their rival. RS proved that investment does incur in equilibrium, but by only one 
of the firms. In this preemption equilibrium consumers never benefit from technological improvements, because the price remains at the high marginal cost of the non-adopting firm. Further, they showed that the preemption equilibrium is completely inefficient: to discourage entry of its rival the preempting firm adopts new technologies so frequently that all of its profits (and thus all social surplus) is completely dissipated. Their result implies that leapfrogging is incompatible with Bertrand price competition.

Though RS stressed that their investment preemption result was "narrow in that it need not hold for other market structures" their analysis "suggests a broader research agenda exploring market structure dynamics" to answer questions such as "Under what conditions do other equilibrium patterns emerge such as action-reaction (Vickers, 1986) or waves of market dominance in which the identity of the market leader changes with some adoptions but not others?" (p. 258).

Giovannetti (2001) advanced the literature by showing that a particular type of leapfrogging — alternating adoptions — can be an equilibrium outcome in a discrete time duopoly model of Bertrand price competition under assumptions that are broadly similar to RS. Though Giovannetti did not cite or specifically address RS's work, he showed that both preemption and alternating adoptions can be equilibrium outcomes depending on the elasticity of demand.

Giovannetti's analysis was done in the context of a game where firms make simultaneous investment decisions, whereas RS modeled the investment choices as an alternating move game. The alternating move assumption seems to be a reasonable way to approximate decisions made in continuous time, where it is unlikely that two firms would be informed of a new technological innovation and make investment decisions at precisely the same instant. However, the change 
in timing assumptions could have significant consequences, since Theorem 1 of RS shows that preemption is the only equilibrium in the continuous time limit of a sequence of discrete-time alternating move investment games as the time between moves tends to zero. RS conjectured that whether firms move simultaneously or alternately makes no difference with respect to the conclusion that preemption is the unique equilibrium of the continuous time limiting game. "We believe the same limit holds if the firms move simultaneously in each stage of the discrete games in the definition. The alternating move structure obviates examining mixed strategy equilibria for some subgames of sequence of discrete games." (RS, p. 255).

Giovannetti's finding that an equilibrium with alternating investments is possible if firms move simultaneously suggests that Riordan and Salant's conjecture is incorrect. Giovannetti did not consider whether his results hold if firms move alternately rather than simultaneously, or whether leapfrogging is sustainable in the continuous time limit. Further neither Giovannetti nor RS considered uncertain technological progress and whether this would affect their conclusions: both assumed that the state of the art production cost declines deterministically over time. Stochastic technological change could create investment opportunities that could upset the preemption equilibrium and lead to more complex adoption dynamics. In particular, deterministic technological progress rules out the possibility of drastic innovations in the sense of Arrow (1962), i.e. discontinuous improvements in technology. Riordan and Salant conjectured that the preemption result was a robust conclusion that would continue to hold in the presence of drastic innovations: "We conjecture that there exists an equilibrium adoption pattern featuring increasing dominance and rent dissipation quite generally. The heuristic reason is the standard one (Gilbert and Newbery, 
1982, Vickers, 1986) that the leading firm always has a weakly greater incentive to preempt to protect its incumbent profit flow." (RS, p. 257).

We characterize the set of Markov perfect equilibria (MPE) payoffs — from both pure and mixed (behavioral) strategies — of a dynamic duopoly model of Bertrand price competition with stochastic technological progress — under both simultaneous and alternating move assumptions (including stochastic alternating move versions of the game). We provide a unifying framework that reconciles the conflicting results of Giovannetti and Riordan and Salant, and by allowing for stochastic technological progress we also study a much wider range of environments than either of these analyses were able to consider. In particular, our analysis of firm behavior and industry dynamics can accommodate drastic innovations that Arrow (1962) contemplated. Similar to the result of Routledge (2010) in the static context, we establish existence of equilibria in the dynamic Bertrand investment game.

We show that rent dissipating investment preemption will not be an equilibrium outcome if any of the three key assumptions (deterministic technological progress, alternating moves, continuous time) is removed, contrary to RS's conjectures. Instead, we show that complex patterns of dynamic investment competition are supported, with leapfrogging occurring in many other forms than simple patterns of deterministically alternating investments of Giovannetti (2001). In fact, we show, via numerical calculation of all MPE of example games using the recursive lexicographical search (RLS) algorithm of Iskhakov, Rust and Schjerning (IRS2016), that various types of leapfrogging equilibria are the typical outcome of the Bertrand investment game.

In the simultaneous move version of the game there is a vast multiplicity of equilibria, and 
our characterization the set of all MPE of this game is reminiscent of the Folk Theorem: the convex hull of the set of initial node pay-offs of the game is a triangle, whose vertices include two monopoly pay-offs and the origin — a mixed strategy equilibrium with zero expected pay-offs to both firms. In the monopoly MPE of our game one duopolist never invests and the other does all of the investing and keeps the price equal to the higher marginal cost of production, earning full monopoly profit. However the monopoly equilibrium is different than the investment preemption equilibrium studied by Riordan and Salant (1994). In particular, we prove that the monopoly equilibria are fully efficient whereas preemption equilibria are completely inefficient and dissipate all rent due to an excessively rapid rate of adoption of new technologies by the low cost leader to avoid being leapfrogged by the high cost follower.

When firms invest in an alternating fashion (under deterministic and stochastically alternating move variations), we show that the convex hull of the set of equilibrium pay-offs is a strict subset of the same triangle, and in particular neither the monopoly nor the zero profit mixed strategy MPE are supportable in this case. We provide a sufficient condition for the uniqueness of equilibrium: in the alternating moves specification when technology improves in every time period with probability one, the Bertrand investment game has a unique MPE. This condition is satisfied in RS's and Giovannetti's frameworks where technological progress is deterministic. However, when the probability of no improvement in the state of the art in any single period is sufficiently large, the set of MPE is no longer a singleton and will in general include a large number of equilibria that exhibit various types of leapfrogging.

Besides our analytic characterization of the the extremal equilibrium pay-offs when investment 
decisions are made simultaneously, we use the RLS algorithm to numerically compute all MPE to finite state versions of the Bertrand investment game and calculate the empirical distribution of their efficiency. We find that MPE are typically inefficient due to investments that occur too frequently relative to the social optimum and due to duplicative investments that are a reflection of coordination failures in this game. The most inefficient equilibria are those involving preemption and mixed strategies. However we show that there are also fully efficient equilibria that take the form of asymmetric pure strategy equilibria and include the monopoly equilibria mentioned above.

Though most of the leapfrogging equilibria display some degree of inefficiency due to duplicative investments, the overall efficiency is generally very high in the Bertrand investment games we have solved. Calculating an efficiency score (the ratio of the total surplus achieved by the social planner to the surplus consumers and producers obtain in the duopoly equilibrium), we find that the median efficiency of all equilibria in examples we provide in section 4 is over $95 \%$. Although investment competition in the non-monopoly equilibria of the model does benefit consumers by lowering costs and prices in the long run, it does generally come at the cost of some inefficiency due to coordination failures. However, we provide examples (and thus establish existence) of perfectly coordinated, fully efficient leapfrogging equilibria as well.

In the next section we present our model and summarize the solution method we used to compute all MPE of the game. Section 3 discusses the socially optimal investment strategies and solves the social planner's problem. We present our main results in section 4, and section 5 concludes. 


\section{The Model}

Consider a market consisting of two firms producing an identical good. Assume that the two firms are price setters, have no fixed costs, and can produce the good at a constant marginal cost of $c_{1}$ and $c_{2}$, respectively. Both firms have constant returns to scale production technology, so neither of them ever faces binding capacity constraints.

Under the assumption of inelastic demand, it is well known that Bertrand equilibrium outcome is for the lower cost firm to serve the entire market at a price $p\left(c_{1}, c_{2}\right)$ equal to the marginal cost of production of the higher cost rival, i.e. $p\left(c_{1}, c_{2}\right)=\max \left[c_{1}, c_{2}\right]$. An alternative interpretation of Bertrand price competition that results in the same equilibrium is to view sales in each period as the outcome from a first price auction. In the auction equilibrium the low cost bidder wins by submitting a bid just below the marginal cost of production of its high cost rival. ${ }^{1}$

In the case where both firms have the same marginal cost of production we obtain the classic result that Bertrand price competition leads to zero profits for both firms at a price equal to their common marginal cost of production. Normalizing the market size to one, we can write the

\footnotetext{
${ }^{1}$ As is well known, a pure strategy Nash equilibrium fails to exist in the asymmetric Bertrand duopoly if the price strategy space is "too large", such as if firms can choose from a continuum of possible prices, see e.g. Ried (1993). This technical problem can be rectified by limiting the size of the price strategy space: "Suppose, for example, firms may only quote prices which are integer multiples of some basic currency unit (e.g. one penny). Then, equilibrium will always exist but a simple characterization is no longer possible whenever the opportunity for genuine price competition the most efficient firm arises. Referring again to the duopoly case, the more efficient firm 1 may, on the one hand, serve the entire market by quoting the highest price lower than or equal to its rival, firm 2. On the other hand, it is perfectly possible that in equilibrium total demand is split between the two firms at the lowest price above or equal to $c_{2} . "$ (p. 231). Since the focus of our analysis is on investment dynamics we adopt the standard interpretation of asymmetric Bertrand equilibrium that the low cost firm serves the entire market at a price one penny below the marginal cost of its rival. Further, we ignore the fact that the market price is actually one penny less than the marginal cost of the high cost firm to keep our notation simple.
} 
instantaneous profits of firm 1 as

$$
r_{1}\left(c_{1}, c_{2}\right)= \begin{cases}0 & \text { if } c_{1} \geq c_{2}, \\ c_{2}-c_{1} & \text { if } c_{1}<c_{2},\end{cases}
$$

and the profits for firm $2, r_{2}\left(c_{1}, c_{2}\right)$ are defined symmetrically, so we have $r_{2}\left(c_{1}, c_{2}\right)=r_{1}\left(c_{2}, c_{1}\right)$. We say that an equilibrium has the Bertrand Property whenever the high cost firm earns zero profits and the low cost firm earns positive profits. Of interest is whether the Bertrand Property extends to payoffs in a simple dynamic extension of the classic model of Bertrand price competition that we introduce below.

Our intertemporal extension of Bertrand price competition takes place in discrete time, though we allow the interval between successive periods to be arbitrarily small which allows us to study the MPE of continuous time limiting versions of the game. In each time period $t$ both firms make simultaneous decisions about the prices they will set, and both have the ability to invest in a new production facility (plant) to replace their existing technology. Technological progress drives down the marginal cost of production (while maintaining constant returns to scale), but we assume it is exogenous and stochastic. That is, an improved production technology is something the firms purchase at a commonly known fixed investment cost — it amounts to a binary acquisition decision and is not the result of variable $R \& D$ investments.

Let $c$ denote the current state of the art marginal cost of production, and let $K(c)$ be the cost of investing in the plant that embodies this state of the art production technology. If either one of the firms purchases the state of the art technology, then after a one period lag (constituting the "time to build" the new production facility), the firm can produce at the new marginal cost $c$.

We assume there is no depreciation and no costs of disposal of an existing production plant, or 
equivalently, the disposal costs do not depend on the vintage of the existing plant and are embedded as part of the new investment cost $K(c)$. We allow the fixed investment $\operatorname{cost} K(c)$ to depend on $c$ to capture different technological possibilities. For example, $K^{\prime}(c)<0$ reflects a situation where the cost of a new plant increases as the state of the art marginal cost gets lower. On the other hand, if $K^{\prime}(c)>0$, then technological improvements that lower the marginal cost of production $c$ also lower the cost of building the plant that is capable of producing output at this state of the art marginal cost.

Clearly, if investment costs are too high, then there may be a point at which the potential gains from lower costs of production are insufficient to justify incurring the investment cost $K(c)$. Moreover, when the competition between the duopolists leads to leapfrogging behavior, the investing firm will not be able to capture the entire benefit of lowering its cost of production: some of these benefits will be passed on to consumers in the form of lower prices.

Let $c_{t}$ denote the marginal cost of production under the state of the art production technology at time period $t \in\{0,1,2, \ldots, \infty\}$. Each period $t$ the firms face a simple binary investment decision: firm $j$ can decide not to invest and continue to produce using its existing production facility at the marginal cost $c_{t, j}$. If firm $j$ pays the investment $\operatorname{cost} K\left(c_{t}\right)$ and acquires the state of the art production plant with marginal cost $c_{t}$, then when this new plant comes on line at $t+1$, firm $j$ will be able to produce at the marginal cost $c_{t+1, j}=c_{t}<c_{t, j}$.

We consider both a continuous state and finite state formulation of the game depending on how we specify the stochastic process for the state of the art cost, $c$. If $c$ is a continuous stochastic process, the state space for this model which we denote $S$, is given by the pyramid $S=\left\{\left(c_{1}, c_{2}, c\right)\right.$ : 
$c_{1} \geq c$ and $c_{2} \geq c$ and $\left.0 \leq c \leq c_{0}\right\}$ in $R^{3}$, where $c_{0}>0$ is the initial state, and zero represents the lower bound of the state of the art technology. The choice of lower bound is not essential for any of our results. The Bertrand investment game starts at the apex of the pyramid given by $\left(c_{0}, c_{0}, c_{0}\right)$. In cases where for computational reasons we restrict $c$ to a finite set of possible values in $\left[0, c_{0}\right]$, the "discretized" state space is a finite lattice subset of $S$.

We assume both firms believe that the state of the art technology for producing the good evolves stochastically according to a Markov process with transition density $\pi\left(c_{t+1} \mid c_{t}\right)$. Specifically, suppose that with probability $\pi\left(c_{t} \mid c_{t}\right)$ there is no improvement in the state of the art technology, and with probability $1-\pi\left(c_{t} \mid c_{t}\right)$ technology improves to marginal $\cos t c_{t+1}$ which is a draw from some distribution over the interval $\left[0, c_{t}\right]$. An example of a convenient functional form for such a distribution is the Beta distribution. However, the presentation of the model and neither of our results do not depend on specific functional form assumptions about $\pi$.

The feature of the transition density $\pi$ that turns out to be crucial for the uniqueness of equilibrium is whether $\pi(c \mid c)>0$ for some $c>0$ or not. We single out a special case of strictly monotonic technological progress when $\pi(c \mid c)=0$ for all $c>0$, i.e. the state of art improves in every time period until it reaches the absorbing state where $\pi(0 \mid 0)=1 .^{2}$ Under deterministic technological progress we have $\pi\left(c_{t+1} \mid c_{t}\right)=1$ if $c_{t}>c_{t+1} \geq 0$ and $\pi\left(c_{t+1} \mid c_{t}\right)=0$ if $c_{t}=c_{t+1}$ including the case $c_{t}=c_{t+1}=0$. Thus, deterministic technological progress is strictly monotonic, but not vice versa.

\footnotetext{
${ }^{2}$ Throughout the paper we use $\pi(c \mid c)=0$ to refer to strictly monotonic progress bearing in mind that it only applies for $c>0$.
} 


\subsection{Timing of Moves}

Let $m_{t}$ be a state variable that governs which of the two firms are "allowed" to undertake an investment at time $t$. We will assume that $\left\{m_{t}\right\}$ evolves as an exogenous Markov chain with transition probability $f\left(m_{t+1} \mid m_{t}\right)$ independent of the other state variables $\left(c_{t, 1}, c_{t, 2}, c_{t}\right)$. While it is natural to assume firms simultaneously set their prices, their investment choices may or may not be made simultaneously.

In this paper we analyze two variants of the Bertrand investment game: 1) a simultaneous move game where the firms make their investment choices simultaneously, denoted by $m_{t}=0$, with $f\left(0 \mid m_{t}\right)=1$ (so $m_{t}=0$ with probability 1 for all $t$ ), and 2) alternating move game, with either deterministic or random alternation of moves, but where there is no chance that the firms could ever undertake simultaneous investments (i.e. where $m_{t} \in\{1,2\}$ and $f\left(0 \mid m_{t}\right)=0$ for all $t$ ). Here $m_{t}=1$ indicates a state where only firm 1 is allowed to invest, and $m_{t}=2$ is the state where only firm 2 can invest. Under either the alternating or simultaneous move specifications, each firm always observes the investment decision of its opponent after the investment decision is made. However, in the simultaneous move game, the firms must make their investment decisions based on their assessment of the probability their opponent will invest. In the alternating move game, since only one of the firms can invest at each time $t$, the mover can condition its decision on the investment decision of its opponent if it was the opponent's turn to move in the previous period. The alternating move specification can potentially reduce some of the strategic uncertainty that arises in a fully simultaneous move specification of the game.

We interpret random alternating moves as a way of reflecting asynchronicity of timing of de- 
cisions in a discrete time model that occurs in continuous time models where probability of two firms making investment decisions at the exact same instant of time is zero. In some cases there is a unique equilibrium when players move in an alternating fashion (e.g. Lagunoff and Matsui, 1997) and we provide sufficient conditions for uniqueness in our model.

The timing of events in the model is as follows. At the start of period $t$ each firm knows the costs of production $\left(c_{t, 1}, c_{t, 2}\right)$, and both learn the current values of $c_{t}$ and $m_{t}$. If $m_{t}=0$, then the firms simultaneously decide whether or not to invest. We assume that both firms know each others' marginal cost of production, i.e. there is common knowledge of state $\left(c_{t, 1}, c_{t, 2}, c_{t}, m_{t}\right)$. Further, both firms have equal access to the new technology by paying the investment $\operatorname{cost} K\left(c_{t}\right)$ to acquire the current state of the art technology with marginal cost of production $c_{t}$.

After each firm decides whether or not to invest in the latest technology, the firms then independently and simultaneously set the prices for their products, where production is done in period $t$ with their existing plant. The Bertrand equilibrium price is the unique Nash equilibrium of the simultaneous move pricing stage game. The one period time-to-build assumption implies that even if both firms invest in new plants at time $t$, their marginal $\operatorname{costs} c_{t, 1}$ and $c_{t, 2}$ in period $t$ are unchanged, and enter profit formula (1).

We assume that consumer purchases of the good is a purely static decision, and consequently there are no dynamic effects of pricing for the firms, unlike in the cases of durable goods where consumer expectations of future prices affects their timing of new durable purchases as in Goettler and Gordon (2011). Thus in our model, the pricing decision is given by the simple static Bertrand equilibrium in every period. The only dynamic decision is firms' investment decisions. 


\subsection{Solution concept}

Assume that the two firms are expected discounted profit maximizers and have a common discount factor $\beta \in(0,1)$. We adopt the standard concept of Markov-perfect equilibrium (MPE) where the firms' investment and pricing decision rules are restricted to be functions of the current state, $\left(c_{t, 1}, c_{t, 2}, c_{t}, m_{t}\right)$. When there are multiple equilibria in this game, the Markovian assumption also restricts the "equilibrium selection rule" to depend only on the current value of the state variable. The firms' pricing decisions only depend on their current production costs $\left(c_{t, 1}, c_{t, 2}\right)$ in accordance with the static Bertrand equilibrium. However, the firms' investment decisions also depend on the value of the state of the art marginal cost of production $c_{t}$ and the designated mover $m_{t}$.

Definition 1. A Stationary Markov Perfect Equilibrium of the duopoly investment and pricing game consists of a pair of strategies $\left(P_{j}\left(c_{1}, c_{2}, c, m\right), p_{j}\left(c_{1}, c_{2}\right)\right), j \in\{1,2\}$ where $P_{j}\left(c_{1}, c_{2}, c, m\right) \in[0,1]$ is firm $j$ 's probability of investing and $p_{j}\left(c_{1}, c_{2}\right)=\max \left[c_{1}, c_{2}\right]$ is firm $j$ 's pricing decision. The investment rules $P_{j}\left(c_{1}, c_{2}, c, m\right)$ must maximize the expected discounted value of firm j's future profit stream taking into account the investment and pricing strategies of its opponent.

We allow the investment strategies of the firms to be probabilistic to allow for the possibility of mixed strategy equilibria. To derive the functional equations characterizing a stationary Markovperfect equilibrium, suppose the current state is $\left(c_{1}, c_{2}, c, m\right)$, i.e. firm 1 has a marginal cost of production $c_{1}$, firm 2 has a marginal cost of production $c_{2}$, and the marginal cost of production using the current best technology is $c$, and $m$ denotes which of the firms has the right to make a move and invest (or both if $m=0$ ). The firms' value functions $V_{j}, j=1,2$ take the form

$$
V_{j}\left(c_{1}, c_{2}, c, m\right)=\max \left[v_{I, j}\left(c_{1}, c_{2}, c, m\right), v_{N, j}\left(c_{1}, c_{2}, c, m\right)\right]
$$


where, when $m=0, v_{N, j}\left(c_{1}, c_{2}, c, m\right)$ denotes the expected value to firm $j$ if it does not invest in the latest technology, and $v_{I, j}\left(c_{1}, c_{2}, c, m\right)$ is the expected value to firm $j$ if it invests. However, when $m \in\{1,2\}$, the subscripts $N$ and $I$ refer to whether an investment is made in period $t$ by the firm $m$, who has the right of move. When $m=1$ (firm 1 has the right to invest), $v_{I, 1}\left(c_{1}, c_{2}, c, 1\right)$ and $v_{N, 1}\left(c_{1}, c_{2}, c, 1\right)$ denote the expected values to firm 1 from investing and not investing. When $m=2$ (firm 2 has the right to invest), $v_{I, 1}\left(c_{1}, c_{2}, c, 2\right)$ and $v_{N, 1}\left(c_{1}, c_{2}, c, 2\right)$ denote the expected values to firm 1 from the scenarios when firm 2 makes the investment or does not make the investment.

The formula for the expected profits associated with not investing is given by:

$$
v_{N, j}\left(c_{1}, c_{2}, c, m\right)=r_{j}\left(c_{1}, c_{2}\right)+\beta E V_{j}\left(c_{1}, c_{2}, c, m, 0\right)
$$

where $E V_{j}\left(c_{1}, c_{2}, m, c, 0\right)$ denotes the conditional expectation of firm $j$ 's next period value function $V_{j}\left(c_{1}, c_{2}, c, m\right)$ given that it does not invest this period (represented by the last 0 argument in $E V_{j}$ ), conditional on the current state $\left(c_{1}, c_{2}, c, m\right)$.

The formula for the expected profits associated with investing is given by

$$
v_{I, j}\left(c_{1}, c_{2}, c, m\right)=r_{j}\left(c_{1}, c_{2}\right)-K(c)+\beta E V_{j}\left(c_{1}, c_{2}, c, m, 1\right)
$$

where $E V_{j}\left(c_{1}, c_{2}, c, m, 1\right)$ is firm $j$ 's conditional expectation of its next period value function given that it invests (the last argument is 1$)$, conditional on $\left(c_{1}, c_{2}, c, m\right)$.

Let $P_{1}\left(c_{1}, c_{2}, c, m\right)$ be firm 2's belief about the probability that firm 1 will invest in state is $\left(c_{1}, c_{2}, c, m\right)$. Consider the simultaneous move case $(m=0)$ first. It follows from (2) that

$$
P_{1}\left(c_{1}, c_{2}, c, m\right)=\mathbb{1}\left\{v_{I, 1}\left(c_{1}, c_{2}, c, m\right)>v_{N, 1}\left(c_{1}, c_{2}, c, m\right)\right\}
$$

where $\mathbb{1}\{\cdot\}$ denotes an indicator function, and mixed strategy investment probability arises in the 
case of equality. A similar formula holds for $P_{2}\left(c_{1}, c_{2}, c, m\right)$.

The Bellman equations for firm 1 in the simultaneous move case are as follows. ${ }^{3}$

$$
\begin{aligned}
v_{N, 1}\left(c_{1}, c_{2}, c\right)= & r_{1}\left(c_{1}, c_{2}\right)+\beta \int_{0}^{c}\left[P_{2}\left(c_{1}, c_{2}, c\right) \max \left(v_{N, 1}\left(c_{1}, c, c^{\prime}\right), v_{I, 1}\left(c_{1}, c, c^{\prime}\right)\right)+\right. \\
& \left.\left(1-P_{2}\left(c_{1}, c_{2}, c\right)\right) \max \left(v_{N, 1}\left(c_{1}, c_{2}, c^{\prime}\right), v_{I, 1}\left(c_{1}, c_{2}, c^{\prime}\right)\right)\right] \pi\left(d c^{\prime} \mid c\right) . \\
v_{I, 1}\left(c_{1}, c_{2}, c\right)= & r_{1}\left(c_{1}, c_{2}\right)-K(c)+\beta \int_{0}^{c}\left[P_{2}\left(c_{1}, c_{2}, c\right) \max \left(v_{N, 1}\left(c, c, c^{\prime}\right), v_{I, 1}\left(c, c, c^{\prime}\right)\right)+\right. \\
& \left.\left(1-P_{2}\left(c_{1}, c_{2}, c\right)\right) \max \left(v_{N, 1}\left(c, c_{2}, c^{\prime}\right), v_{I, 1}\left(c, c_{2}, c^{\prime}\right)\right)\right] \pi\left(d c^{\prime} \mid c\right) .
\end{aligned}
$$

In the alternating move case, the Bellman equations for the two firms lead to a system of eight functional equations for $\left\{v_{N, j}\left(c_{1}, c_{2}, c, m\right), v_{I, j}\left(c_{1}, c_{2}, c, m\right)\right\}$ for $j, m \in\{1,2\}$. The Bellman equations for firm 1 are given below, similar equations for firm 2 are omitted.

$$
\begin{aligned}
v_{N, 1}\left(c_{1}, c_{2}, c, 1\right)= & r_{1}\left(c_{1}, c_{2}\right)+\beta f(1 \mid 1) \int_{0}^{c} \max \left(v_{N, 1}\left(c_{1}, c_{2}, c^{\prime}, 1\right), v_{I, 1}\left(c_{1}, c_{2}, c^{\prime}, 1\right)\right) \pi\left(d c^{\prime} \mid c\right)+ \\
& \beta f(2 \mid 1) \int_{0}^{c} \rho\left(c_{1}, c_{2}, c^{\prime}\right) \pi\left(d c^{\prime} \mid c\right) \\
v_{I, 1}\left(c_{1}, c_{2}, c, 1\right)= & r_{1}\left(c_{1}, c_{2}\right)-K(c)+\beta f(1 \mid 1) \int_{0}^{c} \max \left(v_{N, 1}\left(c, c_{2}, c^{\prime}, 1\right), v_{I, 1}\left(c, c_{2}, c^{\prime}, 1\right)\right) \pi\left(d c^{\prime} \mid c\right)+ \\
& \beta f(2 \mid 1) \int_{0}^{c} \rho\left(c, c_{2}, c^{\prime}\right) \pi\left(d c^{\prime} \mid c\right) \\
v_{N, 1}\left(c_{1}, c_{2}, c, 2\right)= & r_{1}\left(c_{1}, c_{2}\right)+\beta f(1 \mid 2) \int_{0}^{c} \max \left(v_{N, 1}\left(c_{1}, c_{2}, c^{\prime}, 1\right), v_{I, 1}\left(c_{1}, c_{2}, c^{\prime}, 1\right)\right) \pi\left(d c^{\prime} \mid c\right)+ \\
& \beta f(2 \mid 2) \int_{0}^{c} \rho\left(c_{1}, c_{2}, c^{\prime}\right) \pi\left(d c^{\prime} \mid c\right) \\
v_{I, 1}\left(c_{1}, c_{2}, c, 2\right)= & r_{1}\left(c_{1}, c_{2}\right)+\beta f(1 \mid 2) \int_{0}^{c} \max \left(v_{N, 1}\left(c_{1}, c, c^{\prime}, 1\right), v_{I, 1}\left(c_{1}, c, c^{\prime}, 1\right)\right) \pi\left(d c^{\prime} \mid c\right)+ \\
& \beta f(2 \mid 2) \int_{0}^{c} \rho\left(c_{1}, c, c^{\prime}\right) \pi\left(d c^{\prime} \mid c\right) .
\end{aligned}
$$

\footnotetext{
${ }^{3}$ Variable $m=0$ is omitted for clarity. The Bellman equations for firm 2 are omitted for space considerations given that they are analogous to those we present for firm 1.
} 
where

$$
\rho\left(c_{1}, c_{2}, c\right)=P_{2}\left(c_{1}, c_{2}, c, 2\right) v_{I, 1}\left(c_{1}, c_{2}, c, 2\right)+\left[1-P_{2}\left(c_{1}, c_{2}, c, 2\right)\right] v_{N, 1}\left(c_{1}, c_{2}, c, 2\right) .
$$

Note that $P_{2}\left(c_{1}, c_{2}, c, 1\right)=0$, since firm 2 is not allowed to invest when it is firm 1's turn to invest (i.e. when $m=1$ ), and similarly $P_{1}\left(c_{1}, c_{2}, c, c, 2\right)=0$.

The equilibria of the Bertrand investment game with simultaneous moves are characterized by the system of non-linear equations composed of equations (5) and (6) written for every combination of $\left(c_{1}, c_{2}, c\right)$ in the discrete representation of the state space $S$. Similarly, in the alternating moves game, all equilibria are characterized by the system composed of equations (5) and (7) for every combination of $\left(c_{1}, c_{2}, c\right)$ and all values of $m$. In this paper we study the theoretical properties of this system and its solutions, but also solve it for a number of parameter values to provide computed examples and counter-examples.

The key feature of the Bertrand investment game is the directionality of its transitions in the state space $S$ implied by the unidirectional evolution of the state of the art cost $c$ (which can only improve), and the fact that $c_{1}$ and $c_{2}$ will never increase under any feasible strategy. This implies that the Bertrand investment game is in the class of dynamic directional games (DDGs) defined in IRS2016. This paper introduced the recursive lexicographical search (RLS) algorithm that is guaranteed to find all MPE of finite state DDGs provided certain conditions hold. The key conditions are a) there is a finite number of equilibria in every "stage game" (which in the leapfrogging model corresponds to each unique $\left(c_{1}, c_{2}, c\right)$ combination), and $\left.\mathrm{b}\right)$ there is an algorithm that can find all MPE of every stage game. IRS2016 show that both of these conditions hold in the finite state version of the leapfrogging game, so the RLS algorithm can be used to find all MPE of the 
Bertrand investment game. In particular, IRS2016 developed a non-iterative combinatoric algorithm based on the roots of second degree polynomial that finds all of the 1, 3 or 5 possible MPE of every stage game of the simultaneous move version of the investment game, and the 1 or 3 possible MPE of every stage game of the alternating move version.

While RLS can only be applied to compute all MPE in the finite state formulation of the game, the same polynomial equations that characterize the solution to the stage game equilibria applies to both the continuous and the discrete case. We therefore formulate general results that unless explicitly stated applies to both formulations of the game.

Apart from several numerical counter-examples that we provide to the conjecture in Riordan and Salant (1994), we only use the numerical solution of the game to illustrate our general results, which are based on analytic proofs rather than on computed results to specific examples. However the ability to use the RLS algorithm to find all MPE of specific instances of the Bertrand investment game greatly improved our understanding of the structure and the properties of the set of equilibrium outcomes and ultimately facilitated the analytical proofs of the theoretical results we present below.

Before we state our main results in the following sections, we define a symmetric MPE of this game, and discuss the symmetry of equilibrium payoffs.

Definition 2. A Symmetric Markov Perfect Equilibrium of the simultaneous move version of the duopoly investment and pricing game satisfies the following restrictions for any $\left(c_{1}, c_{2}, c\right)$ in the state space:

$$
P_{1}\left(c_{1}, c_{2}, c\right)=P_{2}\left(c_{2}, c_{1}, c\right), \quad v_{N, 1}\left(c_{1}, c_{2}, c\right)=v_{N, 2}\left(c_{2}, c_{1}, c\right), \quad v_{I, 1}\left(c_{1}, c_{2}, c\right)=v_{I, 2}\left(c_{2}, c_{1}, c\right) .
$$


Thus, symmetry implies that the equilibrium behavior of the firms only depends on their cost values $\left(c_{1}, c_{2}\right)$ and not on their "identity" as "firm 1" or "firm 2". We can also define symmetry in the alternating move version of the game analogously. We will show that there exist symmetric MPE in both the alternating and simultaneous move games. However using the RLS algorithm (which can find all MPE in finite state versions of these games) IRS2016 have shown that symmetric MPE constitute only a small fraction of the set of all MPE.

For example, one of the "asymmetric" MPE of the game (whose existence we establish below) is a monopoly equilibrium where only 1 firm (the "monopolist") invests on the equilibrium path whereas its opponent never invests. It is easy to see that this monopoly equilibrium cannot satisfy the definition of symmetry (9) since if firm 1 is the monopolist ${ }^{4}$, then in any state $\left(c_{1}, c_{2}, c\right)$ where it is optimal for the monopolist to invest in the MPE, we have

$$
P_{1}\left(c_{1}, c_{2}, c\right)=1 \neq P_{2}\left(c_{2}, c_{1}, c\right)=0
$$

That is, simply permuting the cost states $\left(c_{1}, c_{2}\right)$ does not change the fact that firm 1 is the monopolist (and thus is the only firm that invests in equilibrium) whereas firm 2 is the "follower" and never invests. Thus in an asymmetric equilibrium the two firms have "identities" and simply permuting the cost values does not lead the two firms to behave the same way or to earn the same discounted profits from investing or not investing.

Of course there is a wider notion of symmetry in terms of the set of equilibrium payoffs. The payoff set at any symmetric point in the state space, $\left(c^{\prime}, c^{\prime}, c\right)$ for $c^{\prime} \in\left[c, c_{0}\right]$ will itself be a sym-

\footnotetext{
${ }^{4}$ We use the term "monopolist" here and below to label the firm that makes the investments in the MPE equilibrium of the game that exhibits the monopoly, i.e. only one firm makes all the investments while the other firm never invests. This should be differentiated from the monopoly solution for the optimal time of investments problem considered in the next section, see Lemma 1.
} 
metric subset of $R_{+}^{2}$, the set of all possible pairs of payoffs to the two firms in any possible MPE of the game starting from $\left(c^{\prime}, c^{\prime}, c\right)$. This is a consequence of the overall arbitrariness (and hence symmetry) in terms of how we label the two firms as "firm 1" and "firm 2". For example if we can find one monopoly equilibrium of the simultaneous move game where firm 1 is the monopolist and firm 2 is the follower, it is clear that there will also be a symmetric monopoly equilibrium where firm 2 is the monopolist and firm 1 is the follower. Though neither of these monopoly MPE are symmetric in themselves for the reason discussed above, it is clear that we will have overall symmetry in the set of MPE payoffs about the "diagonal" points in the state space, $\left(c^{\prime}, c^{\prime}, c\right)$.

\section{Socially optimal production and investment}

We assess the efficiency of the Bertrand investment outcomes relative to a social planning benchmark that maximizes total expected discounted consumer and producer surplus. In a dynamic model, the planner has to account for the costs of investment in new technologies. Since the production technology has constant returns to scale the planner will only operate a single plant. Thus, the duopoly equilibrium can be inefficient due to duplicative investments but we will show that inefficiency manifests itself through inefficient timing of investments as well.

We assume that consumers have quasi-linear preferences so the surplus they receive from consuming the good at a price of $p$ equals their income net of $p$. The social planning solution entails selling the good at the marginal cost of production and adopting an efficient investment strategy that minimizes the expected discounted costs of production. Let $c_{1}$ be the marginal cost of production of the current production plant, and let $c$ be the marginal cost of production of the current state 
of the art production process, which we continue to assume evolves as an exogenous Markov process with transition probability $\pi\left(c^{\prime} \mid c\right)$ whose evolution is beyond the purview of the planner. Let $C\left(c_{1}, c\right)$ be the smallest present discounted value of costs of investment and production when the plant operated by the planner has marginal $\cos t c_{1}$ and the state of the art technology has a marginal cost of $c \leq c_{1}$. The minimization occurs over all feasible investment and production strategies, but subject to the constraint that the planner must produce enough in every period to satisfy the unit mass of consumers in the market. We have

$$
C\left(c_{1}, c\right)=\min \left\{c_{1}+\beta \int_{0}^{c} C\left(c_{1}, c^{\prime}\right) \pi\left(d c^{\prime} \mid c\right), c_{1}+K(c)+\beta \int_{0}^{c} C\left(c, c^{\prime}\right) \pi\left(d c^{\prime} \mid c\right)\right\}
$$

where the first component corresponds to the case when investment is not made, and cost $c_{1}$ is carried in the future, and the second component corresponds to the case when new state of the art $\operatorname{cost} c$ is acquired for additional expense of $K(c)$.

It follows that the optimal investment strategy takes the form of a cutoff rule where it is optimal to invest in the state of the art technology if the current cost $c_{1}$ is above a cutoff threshold $\bar{c}_{1}(c)$. Otherwise the drop in expected future operating costs is not sufficiently large to justify undertaking the investment so it is optimal to produce the good using the existing plant with marginal $\operatorname{cost} c_{1}$. The cutoff rule $\bar{c}_{1}(c)$ is the indifference point in (11), and thus it is the solution to the equation

$$
K(c)=\beta \int_{0}^{c}\left[C\left(\bar{c}_{1}(c), c^{\prime}\right)-C\left(c, c^{\prime}\right)\right] \pi\left(d c^{\prime} \mid c\right)
$$

if it exists, and $\bar{c}_{1}(c)=c_{0}$ otherwise. $^{5}$

We have implicitly assumed that the cost of investment $K(c)$ is not prohibitively high, so that

\footnotetext{
${ }^{5}$ In problems where the support of $\left\{c_{t}\right\}$ is a finite set, the cutoff $\bar{c}_{1}(c)$ is defined as the smallest value of $c_{1}$ in the support of $\left\{c_{t}\right\}$ such that $K(c)>\beta \int_{0}^{c}\left[C\left(c_{1}, c^{\prime}\right)-C\left(c, c^{\prime}\right)\right] \pi\left(d c^{\prime} \mid c\right)$.
} 
the planner would always want to invest in a new technology. Theorem 1 provides a bound on the cost of investment for investment to occur under the socially optimum solution.

Theorem 1 (Necessary and sufficient condition for investment by the planner). Let the current costs be $\left(c_{1}, c\right)$. Investment (in the current period or some time in the future) is socially optimal if and only if there exists $c^{\prime} \in[0, c]$ in the support of the Markov process of the state of the art marginal cost such that

$$
\frac{\beta\left(c_{1}-c^{\prime}\right)}{1-\beta}>K\left(c^{\prime}\right)
$$

The proof of Theorem 1, and all subsequent are in Appendix A. The condition under which it is socially optimal to invest plays a central role when we analyze the duopoly investment dynamics in section 4.

Assumption 1. We will say that the investment costs are not prohibitively high, or that investment is socially optimal if the condition (13) in Theorem 1 holds with a value $c_{1}$ equal to the smaller of the marginal costs of production of the two firms in the Bertrand investment game.

As we will prove in the next section, the Bertrand investment game with simultaneous moves supports a monopoly outcome, i.e. the MPE which is characterized by the fact that one firm makes all the investments while the other remain inactive. The following lemma establishes the efficiency of a monopoly outcome, which is useful for what follows in the next section.

Lemma 1 (Social optimality of monopoly solution). The socially optimal investment policy is identical to the profit maximizing investment policy of a monopolist who faces the same discount 
factor $\beta$ and the same technological process $\left\{c_{t}\right\}$ with transition probability $\pi$ as the planner, assuming that the highest price the monopolist can charge is $c_{0}$, the initial value of the state of the art production technology.

Proof. Since the monopolist is constrained to charge a price no higher than $c_{0}$ every period, it follows that the monopolist maximizes expected discounted value of profits by adopting the same cost-minimizing production and investment strategy as the planner.

\section{Duopoly Investment Dynamics}

We are now in position to solve the model of Bertrand duopoly investment and pricing and characterize the stationary Markov Perfect equilibria of this model. As mentioned above, we used the RLS algorithm from IRS2016 to compute all MPE of the Bertrand investment game. These computations facilitated the illustrative examples below. However the majority of our results are based on analytical proofs of the general properties of the equilibria of this game.

In our analysis we consider the case where the support of state of the art cost $c$ is the entire interval $\left[0, c_{0}\right]$, where $c_{0}>0$ is the initial value of the marginal cost of production at the start of the game at $t=0$. When the state space in continuous, the set of all possible MPE is huge there are at least a continuum of MPE, and if we allow equilibrium selection rules to be Lebesgue rather than Borel-measurable functions, then the cardinality of MPE will be at least $\aleph_{2}$, i.e. the cardinality of the power set of the reals (which of course is strictly greater than the cardinality of the reals, $\aleph_{1}$ ). If we restrict the support of $\left\{c_{t}\right\}$ to be a finite subset of $\left[0, c_{0}\right]$ which implies that the state space of the game is also a finite set and if we restrict attention to deterministic equilibrium 
selection rules, then there will be only a finite number of possible MPE. However IRS2016 show the number of possible MPE grow exponentially fast in the number of states, resulting in a huge (though finite) number of MPE. We conjecture that as the support of a discretized version of the Markov process $\left\{c_{t}\right\}$ becomes dense in the full interval $\left[0, c_{0}\right]$ the finite set of MPE of the finite state version of the game will converge to a dense subset of the set of all MPE of the game where the support of $c$ is the full interval $\left[0, c_{0}\right]$.

Given the large number of possible equilibria, it is therefore impossible to characterize all of them. Instead we focus on characterizing the extremal equilibria i.e. the set of equilibria for which the convex hull of these payoffs includes all possible equilibrium payoffs at the apex of the state space $\left(c_{0}, c_{0}, c_{0}\right)$, which is the initial starting point of the game. In Theorem 2 below, we show that in the case where the firms move simultaneously, there are three extremal equilibria: two monopoly equilibria ${ }^{6}$ and a symmetric zero profit mixed strategy equilibrium (SZP-MSE). We show that the convex hull generated by the payoffs from these three equilibria is a triangle that contains all possible equilibrium payoffs to the game.

After analyzing the simultaneous move game we turn to an analysis of the alternating move game, i.e. where only one of the firms can make an investment decision at any given point in time. We show that the set of terminal node payoffs of the game is a strict subset of the triangle: i.e. neither the monopoly equilibria nor the zero profit mixed strategy equilibria are no longer supported when the firms' investment decisions are made asynchronously.

\footnotetext{
${ }^{6}$ Note that the monopoly equilibria we characterize below are not the preemption equilibrium of Riordan and Salant (1994). In contrast to their rent dissipation result, monopoly profits in our model are positive and are equal to the maximum possible profits subject to the limit on price, and by Lemma 1 the monopoly outcome is efficient.
} 


\subsection{Equilibria of the Simultaneous Move Game}

Proving its existence of SZP-MSE is surprisingly challenging. We have found that the SZP-MSE exists in every game we numerically solved using either RLS algorithm (for discretized state space) or an additional special-purpose continuous state algorithm that constructs the mixed strategy equilibrium recursively. However these calculations only establish the existence of the zero profit mixed strategy equilibrium in the specific examples we calculated: it does not constitute a general proof of existence. In Theorem 2 below we provide some higher level sufficient conditions that enable us to prove the existence of the zero-profit mixed strategy equilibrium in the case where the state space is continuous. However these sufficient conditions are stronger than necessary and we conjecture that this equilibrium exists under much weaker conditions including in finite state versions of the game. In order not to burden the reader with technicalities, we present the assumptions we impose to establish the existence of this equilibrium after stating our main result in Theorem 2 below.

Theorem 2 (Equilibrium payoffs in the simultaneous move game). If investments are socially optimal (in the sense of Assumption 1) at the initial point $\left(c_{0}, c_{0}, c_{0}\right) \in S$ of the state space of the Bertrand investment and pricing game with simultaneous moves, the following hold:

1. No investments by both firms in all states $\left(c_{1}, c_{2}, c\right) \in S$ is not supported in any of the MPE equilibria of the game;

2. There are two fully efficient "monopoly" equilibria in which either one or the other firm makes all the investments and earns maximum feasible profit while their opponent earns zero profits; 
3. Under Assumption 2, Assumption 3 and Assumption 4 (stated below), there exists a unique symmetric zero profit mixed strategy equilibrium (SZP-MSE) that results in zero expected payoffs to both firms in the subgames starting at all diagonal states $\left(c, c, c^{\prime}\right) \in S$ with $c^{\prime} \in$ $[0, c]$.

4. The convex hull of the set of the expected discounted equilibrium payoffs to the two firms in all MPE of the simultaneous move game at the apex $\left(c_{0}, c_{0}, c_{0}\right)$ is a triangle with vertices $(0,0),\left(0, V_{M}\right)$ and $\left(V_{M}, 0\right)$, where $V_{M}=V_{i}\left(c_{0}, c_{0}, c_{0}\right)$ is the expected discounted payoff of firm $i$ which makes all investments in the monopoly equilibrium.

When we refer to "symmetric zero profit mixed strategy equilibrium" (SZP-MSE) it is with the qualification that there are subsets of the state space where neither firm finds it optimal to invest, and there may exist subsets where there are pure strategy equilibria with only one firm investing. For example, it is easy to show that at the edge states - states of the form $\left(c_{1}, c, c\right)$ and $\left(c, c_{2}, c\right)$ — the only possible MPE involves no investment by either firm. Thus, the SZP-MSE will always have a non-empty no investment region - a band of states bordering the edge states where the cost of investment outweighs any gains from leapfrogging the rival firm, making it uneconomic for either firm to invest in these regions. For example there will always be a non-empty no investment region in the bottom layer of the game where $c=0$, because it is easy to see that neither firm can profit from investing in any state $\left(c_{1}, c_{2}, 0\right)$ where $\beta c_{1} /(1-\beta)<K(0)$ or $\beta c_{2} /(1-\beta)<K(0)$, see Lemma A.3 in the Appendix.

We note that except for Statement 3, all of the other Statements of Theorem 2 hold regardless of whether the support of the process $\left\{c_{t}\right\}$ for state of the art cost of production is a finite set 
or the entire interval $\left[0, c_{0}\right]$. The properties of the two monopoly equilibria are clear and easy to understand, however the structure of the SZP-MSE is surprisingly complex and the nature of this equilibrium depends on the assumptions we make about $\pi\left(c^{\prime} \mid c\right), \beta$, and $K(c)$ that we state below. It is tempting to conjecture that the extension of the key property of static Bertrand price competition — namely that the high cost firm always earns zero profits — also holds in the dynamic extension of Bertrand competition. However this conjecture turns out not to be true: we provide counterexamples where $v_{1}\left(c_{1}, c_{2}, c\right)>0$ even though $c_{1}>c_{2}$, i.e. the high cost firm can earn positive expected profits in certain subsets of the state space in the SZP-MSE. In the examples we have calculated, this happens in a pure strategy region where the high cost firm invests and the low cost firm does not invest. However in most of our numerically calculated equilibria we have found that as long as the investment cost $K(c)$ is not too low, then the SZP-MSE will have the Bertrand property i.e. $v_{1}\left(c_{1}, c_{2}, c\right)>0$ when $c_{1}<c_{2}$ and $v_{1}\left(c_{1}, c_{2}, c\right)=0$ when $c_{1} \geq c_{2}$ (similar results hold for $v_{2}$ due to the symmetry of the equilibrium). When the cost of investing is sufficiently high, there can also exist regions of the state space where there is a pure strategy equilibrium where the low cost firm invests and the high cost firm doesn't. In these regions the Bertrand property will hold, i.e. the high cost firm earns zero expected profits whereas the low cost firm earns positive expected profits.

If $\pi(c \mid c)$ decreases in the state of the art cost $c$ (so that technological progress "slows" as the state of the art cost $c$ improves over time), then the following property, which we term antipreemption holds: the high cost firm has a higher probability of investing in the mixed strategy equilibrium than the low cost leader. In the appendix we prove that this result holds in the end 
game where $c=0$, but we show below that in higher stages of the game the result may not hold if $\pi(c \mid c)$ is an increasing function of $c$, so that as technology improves the chances of further progress increase. We have found examples where the high cost follower has a lower probability of investment than the low cost leader in this case, something we refer to as quasi-preemption.

The key property of the SZP-MSE in Statement 3 that appears to hold regardless of whether the support of $\pi\left(c^{\prime} \mid c\right)$ for the state of the art cost is continuous or discrete, and regardless of the properties of $K(c)$ and $\pi\left(c^{\prime} \mid c\right)$ is that in all diagonal states $(\bar{c}, \bar{c}, c)$ expected profits are zero:

$$
v_{1}(\bar{c}, \bar{c}, c) \equiv \max \left[v_{N, 1}(\bar{c}, \bar{c}, c), v_{I, 1}(\bar{c}, \bar{c}, c)\right]=v_{2}(\bar{c}, \bar{c}, c) \equiv \max \left[v_{N, 2}(\bar{c}, \bar{c}, c), v_{I, 2}(\bar{c}, \bar{c}, c)\right]=0
$$

Another key property of the SZP-MSE is the threshold property: for each $c$ there is a threshold $\bar{c}(c)>c$ such that if $c_{1} \geq \bar{c}(c)$ and $c_{2} \geq \bar{c}(c)$ then both firms play mixed strategies, and the Bertrand property holds in this region, i.e. the low cost firm has positive expected profits and the high cost firm has zero expected profits. It is easy to show that the threshold property holds in the end game where $c=0$ : see Lemma A.3 in the Appendix. At higher stages of the game where $c>0$, we establish that the threshold property holds via a transfinite induction argument that relies on the continuity of the state space and the continuity and monotonicity assumptions detailed below.

Figure 1 illustrates the equilibrium regions for a SZP-MSE at two different "slices" of the state space: $c=0$ and $c=1.2245$ in a game where $c_{0}=5$, i.e. the initial cost is 5 . The gray regions are where both firms play mixed strategies, since both $c_{1}$ and $c_{2}$ are above $\bar{c}(c)$ which equals $\bar{c}(0)=.26$ when $c=0$ and $\bar{c}(c)=1.60$ when $c=1.12245$. The black region is a pure strategy region where the low cost firm invests and the high cost firm doesn't. It only exists in the right panel where $c=1.2245$, not in the endgame where $c=0$. The remaining white region where $c_{1} \geq c$ and $c \geq c$ 
Figure 1: Example of equilibrium regions in the SZP-MSE of the simultaneous move game Equilibrium regions, $c=0$

Equilibrium regions, $c=1.22449$
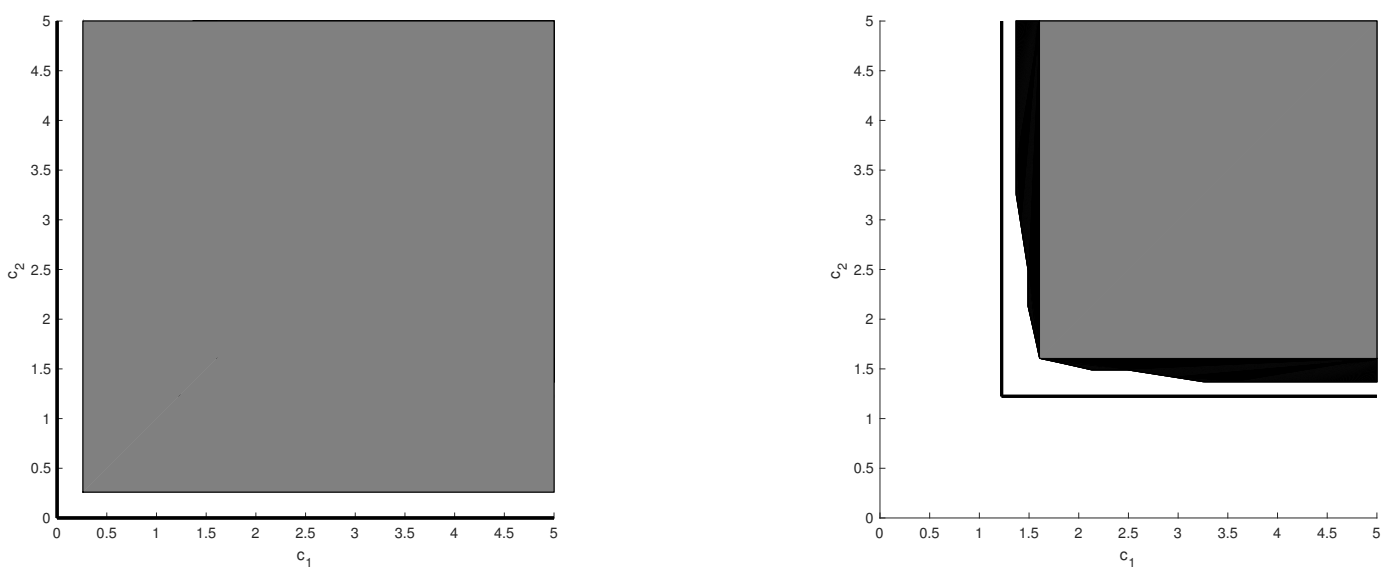

(bounded below and including the heavy black lines that mark the 'edge states' where $c_{1}=c$ or $c_{2}=c$ ) is the no investment region where neither firm invests. As we prove in Lemma A.3, in the end game where $c=0$ there are only two possible equilibrium regions: a mixed strategy region and a no investment region.

We now present and discuss the three additional assumptions that provide sufficient conditions for a SZP-MSE equilibrium to exist.

Assumption 2 (Continuity). Consider the continuous state version of the game. The investment cost function $K(c)$ is a continuous function of $c \in\left[0, c_{0}\right]$. For each $c \in\left[0, c_{0}\right]$, the transition probability $\pi\left(c^{\prime} \mid c\right)$ has at most one discontinuity at $c^{\prime}=c$. The probability of staying at the same state of the art cost, $\pi(c \mid c)$, is a continuous function of $c$. Further $\pi$ is weakly continuous in $c$, that is, for every continuous, bounded function $f: R \rightarrow R$, we have

$$
\lim _{c^{\prime} \rightarrow c} E f\left(c^{\prime}\right)=E f(c)
$$


where

$$
E f(c)=\int_{0}^{c} f\left(c^{\prime}\right) \pi\left(d c^{\prime} \mid c\right)
$$

Assumption 3 (Threshold Monotonicity). The threshold function $\bar{c}(c) \in\left[c, c_{0}\right]$ which bounds the region where both firms play mixed strategies in the SZP-MPE is a continuous non-decreasing funciton of $c$.

Assumption 4 (Value Monotonicity). Let $v_{1}\left(c_{1}, c_{2}, 0\right)$ be firm 1's value in the mixed strategy region of the end-game. For $c_{1}<c_{2}, v_{1}$ is a decreasing function of $c_{1}$ and an increasing function of $c_{2}$. By symmetry, similar results hold for $v_{2}\left(c_{1}, c_{2}, 0\right)$ for $c_{2}<c_{1}$ : it is a non-decreasing function of $c_{1}$.

It would be preferable to prove rather than assume the Value Monotonicity property. Though we believe it does always hold (and we have never found a counterexample to this property in our numerical calculations of equilibrium), we have been unable to find an analytic proof that it must always hold. This is because an increase in $c_{2}$ has two conflicting effects on the value of firm 1 , the low cost leader: 1) a direct effect due to the fact that a rise in $c_{2}$ is effectively an increase in price and therefore its profit, and 2) an indirect, counteracting effect caused by the fact that a rise in $c_{2}$ increases $P_{2}\left(c_{1}, c_{2}, 0\right)$, thus raising the chance that firm 1 will be leap-frogged, and shortening the duration that it can enjoy per period profits of $c_{2}-c_{1}$ while it is the current low cost leader. We can see the conflicting effects in the following expression for $v_{1}\left(c_{1}, c_{2}, 0\right)$

$$
v_{1}\left(c_{1}, c_{2}, 0\right)=\frac{c_{2}-c_{1}}{1-\beta\left[1-P_{2}\left(c_{1}, c_{2}, 0\right)\right]}
$$

Similarly, we have been unable to analytically sign the partial derivatives of the probability of investment by the high cost firm in the mixed strategy region, though both of these partial derivatives 
are always positive in all of the numerically calculated endgame equilibria we have checked. However we can prove that following independence property holds for the low cost firm's probability of investment in the SZP-MSE:

Corollary 2.1 (Independence Property of the SZP-MSE). If $c_{1} \geq \bar{c}(c)$ and $c_{2} \geq \bar{c}(c)$ in the SZPMSE of the simultaneous move game we have

1. If $c_{1} \leq c_{2}, P_{1}\left(c_{1}, c_{2}, c\right)$ is independent of $c_{2}$ and is non-decreasing in $c_{1}$,

2. If $c_{1} \geq c_{2}, P_{2}\left(c_{1}, c_{2}, c\right)$ is independent of $c_{1}$ and is non-decreasing in $c_{2}$.

The independence property is key to our result, and in the proof of Theorem 2 in the Appendix, we derive the following expression for $P_{1}\left(c_{1}, c_{2}, c\right)$ in the mixed strategy region (i.e. where $c_{1} \geq$ $\bar{c}(c)$ and $c_{2} \geq \bar{c}(c)$ and $c_{1} \geq c_{2}$

$$
P_{1}\left(c_{1}, c_{2}, c\right)=1-\frac{K(c)[1-\beta \pi(c \mid c)]}{\beta \pi(c \mid c)\left(c_{1}-c\right)+\beta h_{1}\left(c, c_{1}, c\right)},
$$

where $h_{1}\left(c, c_{1}, c\right)$ is given by

$$
h_{1}\left(c, c_{1}, c\right)=\int_{0}^{c} v_{1}\left(c, c_{1}, c^{\prime}\right) \hat{\pi}\left(d c^{\prime} \mid c\right)
$$

and where $\hat{\pi}\left(c^{\prime} \mid c\right)$ is the restriction of $\pi\left(c^{\prime} \mid c\right)$ to the the interval $[0, c)$, excluding the current state of the art cost $c$. It is obvious from the expression for $P_{1}\left(c_{1}, c_{2}, c\right)$ in equation (18) that $P_{1}$ is independent of $c_{2}$. Via an inductive argument we show that the Value Monotonicity Property holds for all higher levels of the game so that $v_{1}\left(c, c_{1}, c^{\prime}\right)$ is non-decreasing in $c_{1}$ for $c_{1} \geq c>c^{\prime}$. Using equation (18) this implies that $P_{1}\left(c_{1}, c_{2}, c\right)$ is monotonically increasing in $c_{1}$ in the mixed strategy region when $c_{1}<c_{2}$.

Since $P_{1}\left(c_{1}, c_{2}, c\right) \geq 0$ and is monotonically increasing in $c_{1}$, we can derive an expression for the threshold $\bar{c}(c)$ by finding the largest value of $c_{1}>c$ that sets $P_{1}\left(c, c_{1}, c\right)=0$. This is given by 
the implicit function $\bar{c}(c)$ that solves the following equation

$$
K(c)[1-\beta \pi(c \mid c)]=\beta \pi(c \mid c)[\bar{c}(c)-c]+\beta h_{1}(c, \bar{c}, c) .
$$

From equation (20) it is easy to see that $\bar{c}(c)>c$ since we will show in the proof of Theorem 2 that $h_{1}(c, c, c)=0$, and $h_{1}\left(c, c_{1}, c\right)$ is non-decreasing in $c_{1}$. If we totally differentiate this equation and use the Implicit Function Theorem to calculate the derivative $\bar{c}^{\prime}(c)$ we can show that a sufficient condition for the monotonicity of $\bar{c}^{\prime}(c) \geq 0$ to hold is that the following conditions hold:

\section{Assumption 5 (Lower Level Monotonicity Assumptions).}

1. $[1-\beta \pi(c \mid c)] K(c)$ is a differentiable and non-decreasing function of $c$

2. $\pi(c \mid c)$ is a differentiable and non-increasing function of $c$

3. For any $c_{1}>c, h_{1}\left(c, c_{1}, c\right)$ given in equation (19) is a differentiable and non-increasing function of $c$.

Together these lower level monotonicity assumptions in Assumption 5 imply the higher level Threshold Monotonicity Property in Assumption 3. However in order to simplify the proof of Theorem 2 we chose to make the "higher level" assumption on $\bar{c}(c)$ rather than derive it from "lower level" assumptions on the objects, $\pi, K$ and $h_{1}{ }^{7}$

However while the Threshold Monotonicity Property is an appealing and intuitive property that is sufficient to prove the existence of the SZP-MSE, it is not a necessary condition for existence. In fact via numerical calculations, we can show that the SZP-MSE exists even if conditions 1 and 2 on $(1-\beta \pi(c \mid c)) K(c)$ and $\pi(c)$ are violated, resulting in a non-monotonic threshold function $\bar{c}(c)$. For example, Figure 2 illustrates numerically calculated thresholds $\bar{c}(c)$ for the case a) $K(c)=$

\footnotetext{
${ }^{7}$ Note that since $h_{1}$ is itself a "derived object" that results from the equilibrium, its monotonicity in $c$ requires us to show that $v_{1}\left(c_{1}, c_{2}, c\right)$ is non-increasing in $c_{1}$ for $c_{1}<c_{2}$. In fact, our proof of Theorem 2 does inductively establish that this monotonicity property holds for all $c$.
} 
Figure 2: Examples of thresholds $\bar{c}(c)$ for the mixed strategy region of the SZP-MSE
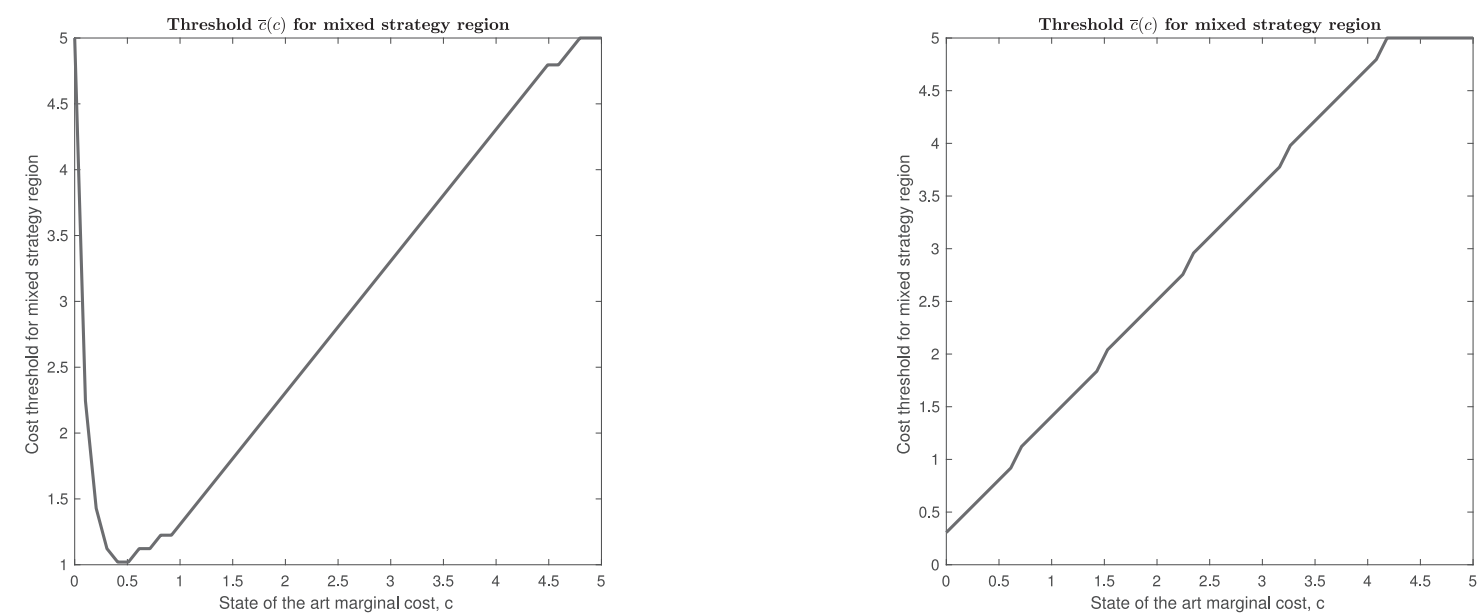

$5.2 /(.18+c)($ left panel) and b) $K(c)=5.2 /(1+.18 c)$ (right panel). Case a) involves investment costs that increase rapidly to a value of $K(0)=260$ as $c \downarrow 0$ whereas in case b) $K(0)=5.2$. Thus in case a) the rapid rise in cost of investment as technology improves chokes off investment and makes it uneconomic for the firms to invest the amount required to reach the limiting zero marginal cost of production technology. It also violates the condition that $[1-\beta \pi(c \mid c)] K(c)$ is a non-decreasing function of $c$, and the failure of this assumption results in a non-monotonic $\bar{c}(c)$ function. Yet, despite this non-monotonicity, we can verify that the SZP-MSE exists and satisifies the properties of Theorem 2 including the Bertrand property (i.e. the high cost firm earns zero expected profits at any point in the state space under the SZP-MSE). The "anti-preemption" property also holds in this case.

The summary of the proof of Theorem 2 is the following. We show that in the SZP-MSE of the overall game there is subsets of states in $S$ where the firms actually do not play mixed strategies. We prove that the stage game MPE, i.e. the set of all possible MPE at any given state of the game, $\left(c_{1}, c_{2}, c\right)$, can be only one of the following four types: 
Type 1 three possible MPE, two of which are pure strategy "anti-coordination" MPEs where one firm invests and the other doesn't, and a third mixed MPE where both firms invest with positive probability

Type 2 a single pure strategy "no investment" MPE where neither firm invests

Type 3 a single pure strategy MPE where the low cost firm invests but the high cost firm doesn't

Type 4 a single pure strategy MPE where the high cost firm invests but the low cost firm doesn't.

As we noted above, the Type 4 equilibria generally only arise when investment costs are sufficiently low. Type 3 and 4 equilibria are not possible in the endgame where $c=0$, and thus only occur at higher stages of the game where $c>0$. The key to the proof of Theorem 2 is to show that Assumption 3 implies that the mixed strategy regions where the Type 1 MPE occur are nested so that as the state of the art cost $c$ transits (potentially stochastically) to any lower cost $c^{\prime}<c$, if the game is in a Type 1 equilibrium at $c$ then the Bellman equations for the firms' values will depend only on values $v_{1}\left(c_{1}, c_{2}, c^{\prime}\right)$ and $v_{2}\left(c_{1}, c_{2}, c^{\prime}\right)$ that are also in the Type 1 region at the lower cost levels $c^{\prime}<c$. By induction, we will be able to show that if the "Bertrand property" holds at all $c^{\prime}<c$ then the Bertrand property holds at $c$ as well. In particular, the Bertrand property implies the key result that both firms earn zero profits on the diagonal states, see equation (14).

The following Corollary provides a sufficient condition to rule out the existence of regions where the anomalous Type 4 equilibria exist. Provided that an intuitive "increasing differences" property holds for the value functions, there can be no regions in the state space where the high cost firm would like to invest but the low cost firm doesn't. This implies in turn that the Bertrand property holds over the entire state space, i.e. the high cost firm's value function is zero in any state $\left(c_{1}, c_{2}, c\right)$ where $c_{2} \geq c_{1}$. 
Corollary 2.2 (Sufficient condition to rule out Type 4 equilibria). Suppose that in the SZP-MSE the value functions satisfy the following increasing differences property

$$
v_{1}\left(c, c_{2}, c\right)-v_{1}\left(c_{1}, c_{2}, c\right) \geq v_{1}\left(c, c_{1}, c\right)-v_{1}\left(c_{1}, c_{1}, c\right) \quad \forall c \leq c_{1} \leq c_{2}
$$

and symmetrically for $v_{2}$. Then in the SZP-MSE there are no Type 4 equilibria and the Bertrand property holds for all $\left(c_{1}, c_{2}, c\right) \in S$.

The increasing difference property (21) is equivalent to submodularity of the value functions, and has an intuitive interpretation. It says that the gain in value to firm 1 from adopting the state of the art production technology is greater if its opponent firm has $\cos t c_{2}$ than it is if its opponent has a lower cost $c_{1} \leq c_{2}$. It is straightforward to prove that this property holds in the endgame, $c=0$, for which we already know no Type 4 equilibria can exist by Lemma A.3. However we have constructed examples where investment costs are sufficiently low that Type 4 equilibria do exist in regions of the state space, and hence the increasing differences property (21) does not hold in all parts of the state space in such games. ${ }^{8}$

Figure 3 illustrates a SZP-MSE that has Type 1, 2 and 3 regions in the state space but no Type 4 regions. The left panel of the figure plots the equilibrium investment probability for firm 1 , $P_{1}\left(c_{1}, c_{2}, c\right)$ where $c=0.83333$ and $K=5.2$ and $\beta=.95$. The region where the Type 1 mixed strategy stage game MPE exist is under the curved region in the upper northeast orthant in the diagram. The Type 2 no investment region occurs along the edges, and the Type 3 pure strategy MPE where the low cost firm invests and the high cost doesn't occurs along a narrow band for $c_{1}$ in the interval $[1.09,1.5]$ and $c_{2}$ is higher than 1.5 .

\footnotetext{
${ }^{8}$ Indeed in the end-game the value functions are submodular on the diagonal states but supermodular in the off-diagonal states in the mixed strategy region.
} 
Figure 3: Example of $\left(P_{1}, v_{1}\right)$ for a simultaneous move SZP-MSE with Type 1, 2 and 3 regions
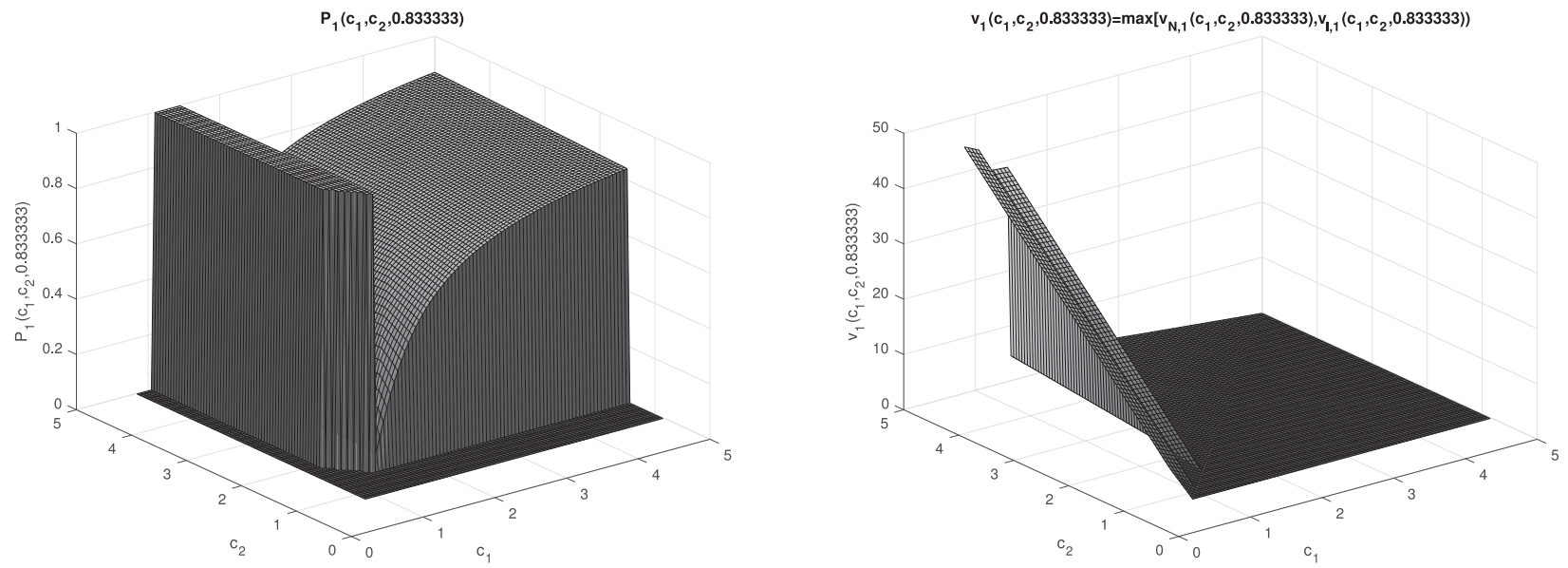

The right hand panel shows the value function for firm 1 in this MPE. The increasing differences property (21) holds in this case, so as predicted by Theorem 2 and Corollary 2.2, we have $v_{1}\left(c_{1}, c_{2}, c\right)=0$ for all $c_{1} \geq c_{2}$ and $v_{1}\left(c_{1}, c_{2}, c\right)>0$ for all $c_{1}<c_{2}$. Note the discontinuities in $v_{1}$ at $c_{1}=1.5$ at the boundary between the Type 1 mixed strategy MPE region and the Type 3 pure strategy region where firm 1 invests and firm 2 doesn't, as well as another discontinuity between the Type 3 region and the Type 2 no investment MPE region, and a third discontinuity along a line where $c_{1}$ is equal to the boundary between the Type 1 mixed strategy region and the Type 2 no investment region where the probability firm 1 invests drops discontinuously to zero.

Figure 4 illustrates the MPE investment probability and value functions for firm 1 in a SZPMSE where investment cost function $K(c)$ is much lower (for example $K(0)=.2$ compared to $K(0)=5.2$ for the equilibrium illustrated in Figure 3) and where $\pi(c \mid c)$ is an increasing rather than decreasing function of $c$. In this equilibrium the increasing differences property (21) fails to hold at all relevant points and as a consequence, the anomalous Type 4 pure strategy regions exist where the high cost firm invests and the low cost firm doesn't. In this region, the high cost firm's 
Figure 4: Example of $\left(P_{1}, v_{1}\right)$ for a simultaneous move SZP-MSE with Type 1, 2 and 4 regions
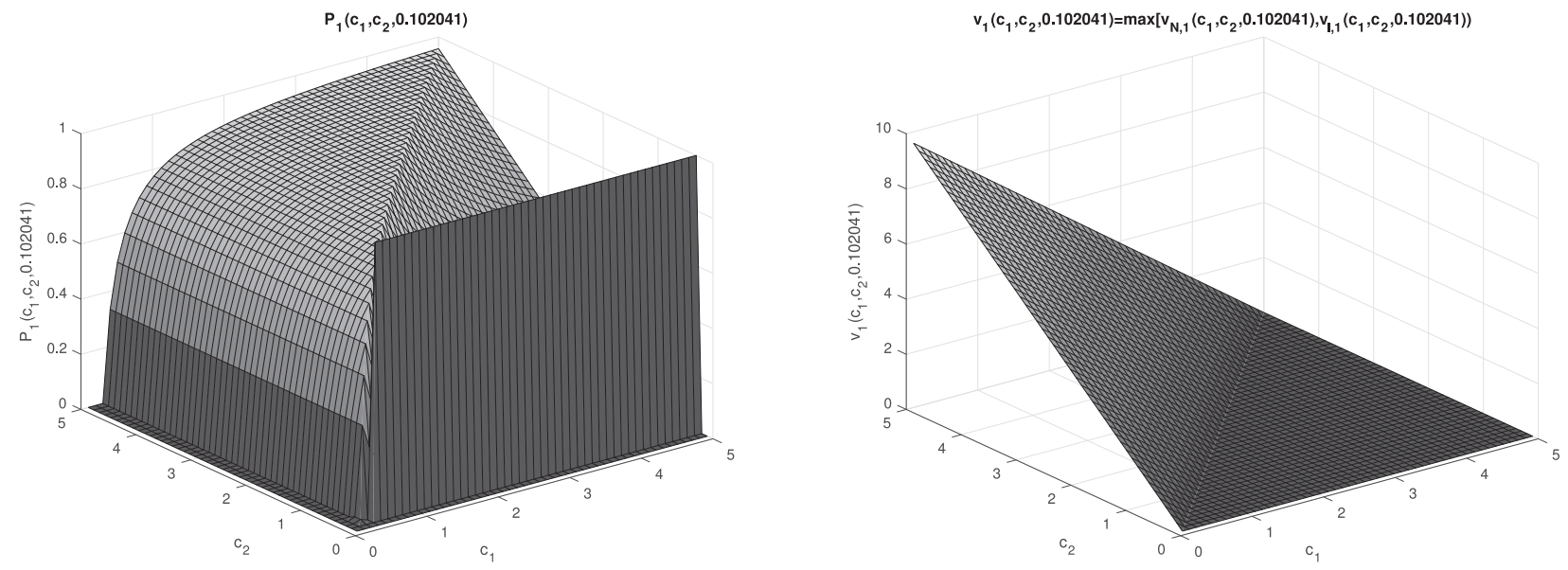

value function is strictly positive, violating the Bertrand property (though the Bertrand property still holds at all diagonal states, so it is not a counterexample to Theorem 2 or Corollary 2.2). For example, $v_{1}\left(c_{1}, c_{2}, c\right)=.0018$ for all $c_{1}>=0.5102$ when $c_{2}=.306$ and $c=.102$. Note also that in the region where $c_{1}>c_{2}, P_{1}\left(c_{1}, c_{2}, c\right)$ is a decreasing function of $c_{1}$, violating the "antipreemption" property, i.e. that the high cost firm should have a higher probability of investing to leapfrog its low cost rival. Note that anti-preemption does hold for the SZP-MSE in Figure 3.

Figure 5 illustrates Theorem 2 by plotting all apex payoffs to the two firms under all possible deterministic equilibrium selection rules in the simultaneous move game where the support of $\left\{c_{t}\right\}$ is the 5 point set $\{0,1.25,2.5,3.75,5\}$. Panel (b) plots the set of payoffs that occur when technological progress is deterministic, whereas panel (a) shows the much denser set of payoffs that occur when technological progress is stochastic. Though there are actually a greater total number of equilibria $(192,736,405)$ under deterministic technological progress, many of these equilibria are observationally equivalent repetitions of the same payoff point which arise due to our treatment of the equilibrium selection rules that only differ off the equilibrium path as distinct. We indicate the 
Figure 5: Initial node equilibrium payoffs in the simultaneous move game
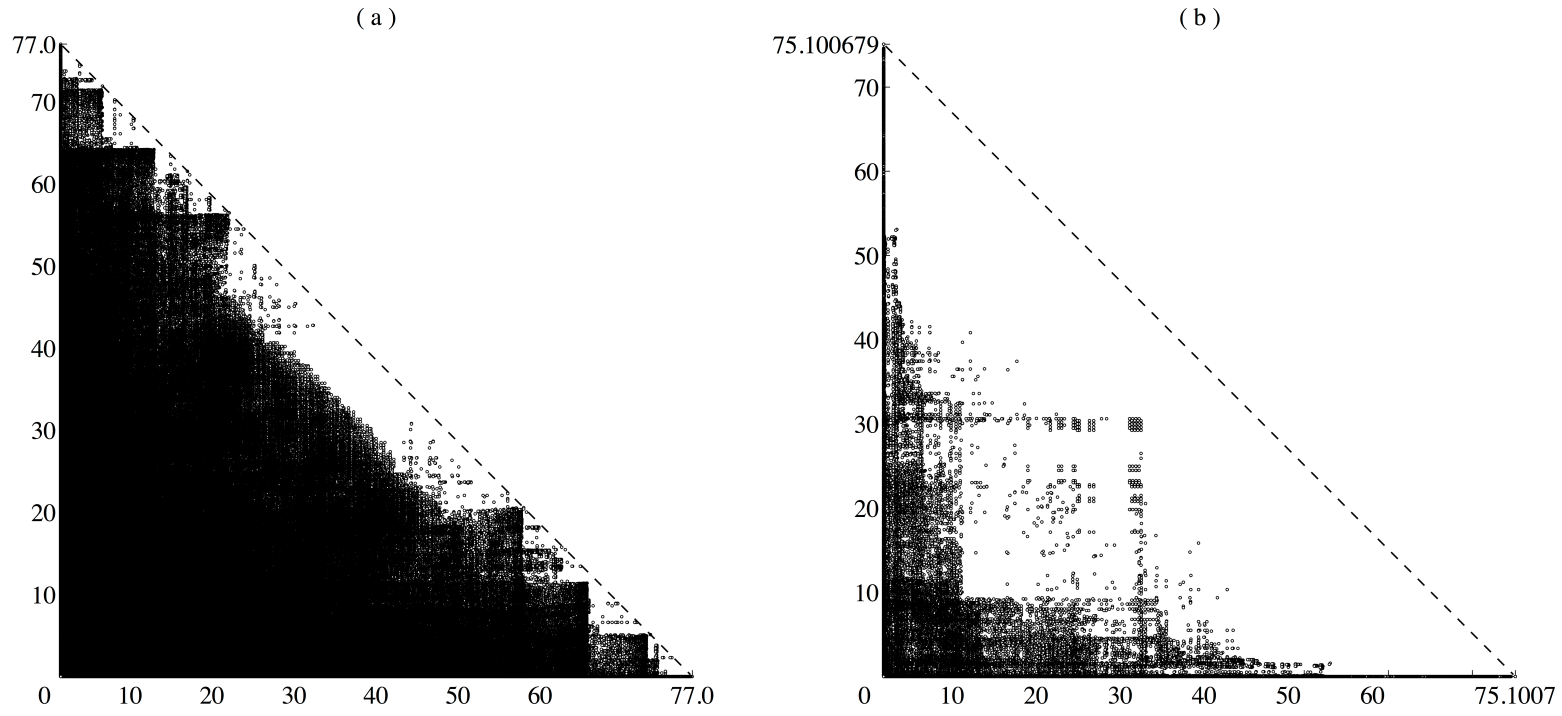

Notes: The panels plot payoff maps of the Bertrand investment game with strictly monotonic random (panel a) and deterministic (panel b) technological improvement. Model parameters are $\beta=0.9512, k_{1}=8.3, k_{2}=1, n_{c}=5$. In the deterministic case the technology deterministically improves one step each time period until it reaches its absorbing state $c=0$. In the stochastic case technology also improves every period until $c=0$, but the new (lower) marginal cost of production $c_{t+1}$ is beta distributed with parameters $a=1.8$ and $b=0.4$ over the interval $\left[0, c_{t}\right]$. Panel (a) displays 1,679,461 distinct points that represent payoffs from 164,295,079 equilibria, panel (b) displays 63,676 distinct payoff from 192,736,405 equilibria of the game.

number of repetitions by the size of the payoff point plotted to be proportional to the number of repetitions. Figure 5 shows that when technology is stochastic there are fewer repetitions and so even though there are actually 28 million fewer equilibria, there are actually a substantially greater number $(1,679,461$ versus 63,676$)$ of distinct payoff points.

Define the leapfrogging equilibria as those where the high cost firm has a positive probability of investing at least once in the support of the equilibrium, and thus a realization of such equilibrium may contain an event when a high cost firm leapfrogs the cost leader. With this definition, leapfrogging equilibria are very typical. We can also show (via the RLS algorithm) that leapfrogging may occur in pure strategy $M P E$ of the either the simultaneous or alternating move versions 
of the game, and further there exist fully efficient leapfrogging MPE. The mixed strategy MPE will generally be inefficient, both due to duplicative investments and due to suboptimally timed investments. We will discuss the issue of efficiency of the MPE in subsection 4.3 below.

\subsection{Equilibria of the Alternating Move Game}

When firms make simultaneous investment decisions in the monopoly equilibrium, the high cost firm has no incentive to deviate from the equilibrium path in which its opponent always invests. However, when the firms move in an alternating fashion, the high cost firm will have an incentive to deviate because it knows that its opponent will not be able to invest at the same time (thereby avoiding the Bertrand investment paradox), and once the opponent sees that the firm has invested, it will not have an incentive to immediately invest to leapfrog for a number of periods until it is once again its turn to invest and there has been a sufficient improvement in the state of the art marginal cost of production. This creates a temptation for each firm to invest and leapfrog their rival that is not present in the simultaneous move game, and the alternating move structure prevents the firms from undertaking inefficient simultaneous investments, though it also generally prevents either firm from being able to time their investments in a socially optimal way.

Theorem 3 (The set of equilibrium payoffs in the Alternating Move Game). If investments are socially optimal (in the sense of Assumption 1) at the apex $\left(c_{0}, c_{0}, c_{0}\right)$ of the state space of the Bertrand investment and pricing game with alternating moves, no investments by both firms is not supported in any of the MPE equilibria of the game. The convex hull of the set of expected discounted equilibrium payoffs to the two firms in all possible MPE equilibria at the apex of the 
alternating move game is a strict subset of the corresponding convex hull of payoffs in the simultaneous move game, i.e. the triangle with vertices defined in Theorem 2.

While it is perhaps not surprising that when firms move in an alternating fashion neither one of them will be able to attain monopoly payoffs in any equilibrium of the alternating move game, Theorem 3 states that a zero expected profit mixed strategy equilibrium payoffs is not sustainable in the alternating move game either. Though it may seem tempting to conclude that mixed strategies can never arise in the alternating move game, we find that both pure and mixed strategy stage game equilibria are possible in the alternating move game. The intuition as to why this should occur is that even though only one firm invests at any given time, when $\pi(c \mid c)>0$ the firms know that there is a positive probability that they will remain in the same state $\left(c_{1}, c_{2}, c\right)$ for multiple periods until the technology improves. The possibility of remaining in the same state implies that the payoff to each firm from not investing depends on their belief about the probability their opponent will invest in this state at its turn.

Thus, by examining the Bellman equations (7) it not hard to see that for firm 1 the value of not investing when it is its turn to invest, $v_{N, 1}\left(c_{1}, c_{2}, c, 1\right)$, depends on $P_{2}\left(c_{1}, c_{2}, c, 2\right)$ when $\pi(c \mid c)>0$. This implies that $P_{1}\left(c_{1}, c_{2}, c, 1\right)$ will depend on $P_{2}\left(c_{1}, c_{2}, c, 2\right)$, and similarly, $P_{2}\left(c_{1}, c_{2}, c, 2\right)$ will depend on $P_{1}\left(c_{1}, c_{2}, c, 1\right)$. This mutual dependency creates the possibility for multiple solutions to the Bellman equations and the firms' investment probabilities and multiple equilibria at various stage games of the alternating move game.

In spite of very large number of MPE we find in the Bertrand investment game, there is a subclass of games for which the equilibrium is unique. 
Theorem 4 (Sufficient conditions for uniqueness). In the dynamic Bertrand investment and pricing game a sufficient condition for the MPE to be unique is that (i) firms move in alternating fashion (i.e. $m \neq 0$ ), and (ii) for each $c$ in the support of $\pi$ we have $\pi(c \mid c)=0$.

Theorem 4 implies that under strictly monotonic technological improvement the alternating move investment game has a unique Markov perfect equilibrium. This is closely related to, but not identical with an assumption of the deterministic technological progress as discussed in section 2. There are specific types of non-deterministic technological progress for which Theorem 4 will still hold, resulting in a unique equilibrium to the alternating move game.

\subsection{Efficiency of equilibria}

We evaluated the efficiency of duopoly equilibria by calculating their efficiency score defined as the ratio of total surplus (i.e. the sum of discounted consumer surplus plus total discounted profits) under the duopoly equilibrium to the maximum total surplus achieved under the social planning solution. ${ }^{9}$ We note that the calculation of efficiency is equilibrium specific and thus its value depends on the particular equilibrium of the overall game that we select. For example, we have already proved that monopoly investment by one of the firms is an equilibrium in the simultaneous move game, provided the cost of investment is not prohibitively high. This implies immediately that there do exist fully efficient MPE in the simultaneous move game. We now show that the nonmonopoly equilibria of either the simultaneous or alternating move investment games are generally

\footnotetext{
${ }^{9}$ It is not hard to show that the sum of discounted expected consumer surplus and discounted expected profits can be calculated from model parameters and the expected discounted cost function $C\left(c_{1}, c_{2}, c\right)$ which we compute in the similar procedure as the value functions in Section 2. Further details are available from authors upon request.
} 
inefficient and this inefficiency is typically due to two sources a) duplicative investments (only in mixed strategy equilibria in the simultaneous move investment game), and b) excessively frequent investments. Note that it is logically possible that inefficiency could arise from excessively infrequent investments and the logic of the Bertrand investment paradox might lead us to conjecture that we should see investments that are too infrequent in equilibrium relative to what the social planner would do. Surprisingly, we find that duopoly investments are generally excessively frequent compared to the social optimum, with preemptive investments (when they arise) representing the most extreme form of inefficient excessively frequent investment in the new technology.

The two panels in the left column in Figure 6 illustrate the set of equilibrium payoffs from all MPE equilibria computed by the RLS algorithm. We compute the efficiency of each of the equilibria, and treating the calculated efficiencies as "data", we plot their empirical distribution in the corresponding panels in the right column in Figure 6.

Panels (a) and (b) in Figure 6 represent an alternating move investment game with deterministic alternations of the right to move and technological progress which is not strictly monotonic, i.e. $\pi(c \mid c)>0$ for some $c$. Consistent with Theorem 3 the set of equilibrium payoffs is a strict subset of the triangle, showing that it is not possible to achieve the monopoly payoffs (corners) or the zero profit mixed strategy equilibrium payoff (origin) in this case. The size of the plotted points indicates the number of repetitions of the payoffs, while the lighter shades indicate higher efficiency equilibria.

We see a clear positive correlation between payoff and efficiency in panel (a) - there is a tendency for the points with the highest total payoffs (i.e. points closest to the line connecting 
Figure 6: Payoff maps and efficiency of MPE in two specifications of the game
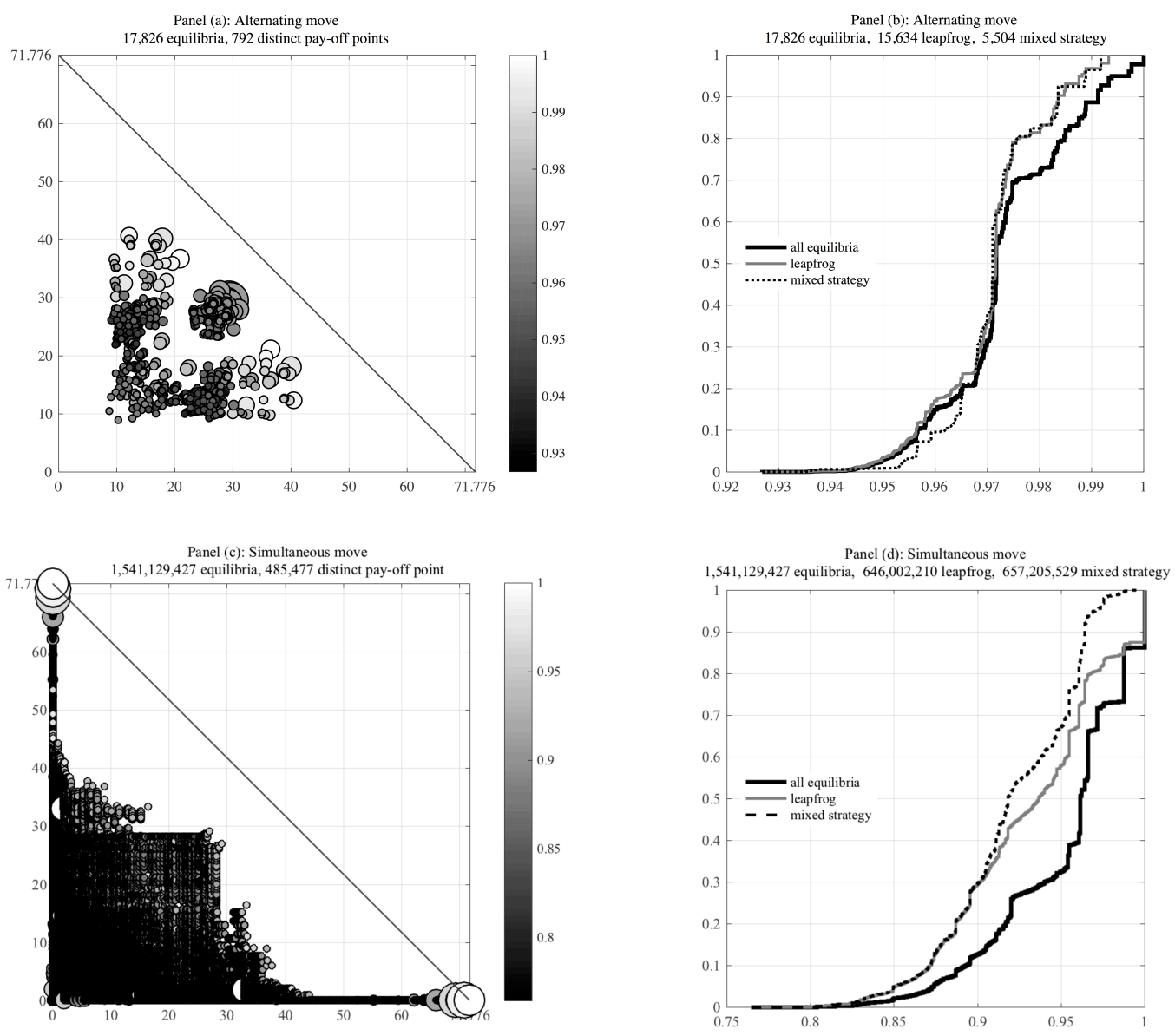

Notes: Panels (a) and (c) plot payoff maps correspondingly for alternating and simultaneous move Bertrand investment and pricing game. Panels (b) and (d) show CDFs of the efficiency measures of the corresponding equilibria. Technological progress in both versions of the game is stochastic: with probability $\pi(c \mid c)=1 /(1+c)$ the state-ofthe-art marginal cost of production remains the same, and with the complement probability it is reduced by one step. Other parameters in both games are $\beta=0.9512, k_{1}=5, k_{2}=0$ and $n_{c}=5$. The alternating move game in panels (a) and (b) has deterministic alternations with $f(1 \mid 1)=f(2 \mid 2)=0, f(2 \mid 1)=f(1 \mid 2)=1$. Leapfrog equilibria are defined as having positive probability to invest by the cost follower along the equilibrium path, mixed strategy equilibria are defined as involving at least one mixed strategy stage equilibrium along the equilibrium path.

the monopoly outcomes) to have higher efficiency measure. The CDFs of the efficiency levels in panel (c) shows that 1) overall efficiency is reasonably high, with the median equilibrium having an efficiency in excess of $97 \%$, and 2) the maximum efficiency of the equilibria involving mixed 
strategies along the equilibrium path is strictly less than $100 \%$.

In panels (c) and (d) of Figure 6 we plot the set of equilibrium payoffs and distribution of efficiency for a simultaneous move investment game under the same technological progress. In accordance with Theorem 2 the monopoly and zero profit outcomes are now present among the computed MPE equilibria of the model. Overall, the equilibria in this game are less efficient compared to the equilibria in the alternating move game displayed in panels (a) and (b), but the tendency of more efficient equilibria to be located closer to the "monopoly" frontier remains. An additional source of inefficiency in the simultaneous move game is duplicative investments, which occur in mixed strategy equilibria. The cumulative distribution plot in panel (d) shows that even though more than $25 \%$ of the equilibria are approaching full efficiency ${ }^{10}$, the mixed strategy equilibria are not among them. Instead, the distribution of their efficiency is stochastically dominated by the distribution of efficiencies in all the equilibria of the game.

Theorem 5 (Inefficiency of mixed strategy equilibria). A necessary condition for efficiency in the dynamic Bertrand investment and pricing game is that only pure strategis are played on the equilibrium path.

Figure 7 establishes the existence of fully efficient leapfrogging equilibria. Panel (a) of Figure 7 plots the set of equilibrium payoffs in a simultaneous move investment game where there are four possible values for state of the art costs $\{0,1.67,3.33,5\}$ and technology improves deterministically. Recall that the payoff points in white are $100 \%$ efficient, so we see that there are a number of other non-monopoly equilibria that can achieve full efficiency. The significance of this finding

\footnotetext{
${ }^{10}$ More specifically, approximatelly $12 \%$ have efficiency of 0.987 and $14 \%$ of equilibria are fully efficient.
} 
Figure 7: Efficiency of equilibria
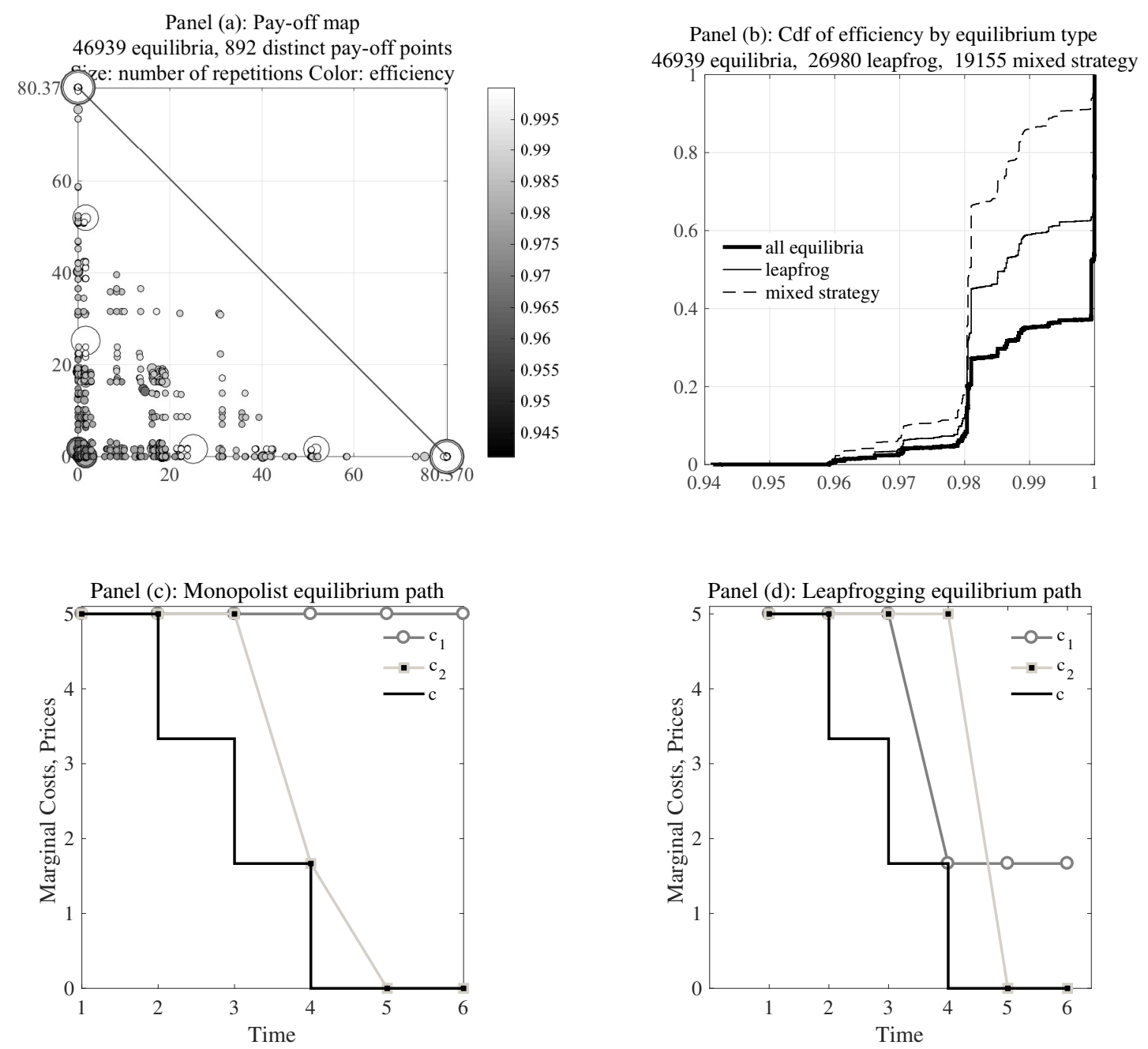

Notes: Panels (a) and (b) plot the apex payoff map and distribution of efficiency for the simultaneous move game. $25.88 \%$ of all equilibria are fully efficient. The most efficient mixed strategy equilibrium in panel (b) has the efficiency index 0.99998 but does not violate Theorem 5. Panel (c) displays the simulated investment profile from a fully efficient "monopoly" equilibrium, while panel (d) displays the example of fully efficient equilibrium that involves leapfrogging.

is that we have shown that it is possible to obtain competitive equilibria where leapfrogging by the firms ensures that consumers receive some of the surplus and benefits from technological progress without a cost in terms of inefficient investment such as we have observed occurs in mixed strategy equilibria of the game where socially inefficient excessive investment results in lower prices to 
consumers but at the cost of zero expected profits to firms. Notice, however, that even the least efficient mixed strategy equilibrium still has an efficiency of $96 \%$, so that in this particular example the inefficiency of various equilibria may not be a huge concern.

Panels (c) and (d) of Figure 7 plot the simulated investment profiles of two different equilibria. Panel (c) shows the monopoly equilibria where firm 2 is the monopolist investor. The socially optimal investment policy is to make exactly two investments: the first when costs have fallen from 5 to 1.67 , and the second when costs have fallen to the absorbing value of 0 . Panel (d) shows the equilibrium realization from a pure strategy equilibrium that involves leapfrogging, yet the investments are made at exact same time as the social planner would do. After firm 1 invests when costs reach 1.67 (consumers continue to pay the price $p_{1}=5$ ), in time period 5 it is leapfrogged by firm 2 who becomes the permanent low cost producer. At this point a "price war" brings the price down from 5 to 1.67, which becomes new permanent level.

We conclude that the leapfrogging equilibria may be fully efficient if investments are made in the same moments of time as the monopolist would invest, but in these equilibria consumers also benefit from the investments because the price decreases in a series of permanent drops.

Lemma 2 (Existence of efficient non-monopoly equilibria). In both the simultaneous move and alternating move investment games, there exist fully efficient non-monopoly equilibria.

Proof. The proof is by example shown in Figure 7. An example of a fully efficient non-monopoly equilibrium when the firms move alternately (in deterministic fashion) can be constructed as well ${ }^{11}$.

\footnotetext{
${ }^{11}$ Let the possible cost states be $\{0,5,10\}$, assume deterministic technological progress, the cost of investing $K=4$, and the discount factor $\beta=0.95$. Then the socially optimal investment strategy is for investments to occur when $c=5$ and $c=10$, and these investments will occur at those states in the unique equilibrium of the game, but where one firm makes the first investment at $c=5$ and the opponent makes the other investment when $c=0$. These investments clearly involve leapfrogging that is also fully efficient.
} 
While we find that efficient leapfrogging occur generically as equilibria in the simultaneous move investment game, the result that there exist efficient leapfrogging equilibria in the alternating move investment game should be viewed as a special counterexample, and that we typically do not get fully efficient leapfrogging equilibria in alternating move games with a sufficiently fine discretization of the state space and when investment costs are "reasonable" in relation to production costs (i.e. when the cost of building a new plant $K(c)$ significantly different from zero). However, due to the vast multiplicity of equilibria in the simultaneous move investment game, we have no basis for asserting that efficient leapfrogging equilibria are any more likely to arise than other more inefficient equilibria.

In summary inefficiency is generally caused by excessive frequency of investment rather than underinvestment. In simultaneous move games we already noted that another source of inefficiency is redundant, duplicative investments that occur only in mixed strategy equilibria. We noted that while mixed strategy equilibria also exist in the alternating move investment game, duplicative simultaneous investments cannot occur by the assumption that only one firm can invest at any given time. Thus, the inefficiency of the mixed strategy equilibria of the alternating move games is generally a result of excessively frequent investment under the mixed strategy equilibrium. However, it is important to point out that we have constructed examples of inefficient equilibria where there is underinvestment relative to the social optimum. Such an example is provided in panel (b) of Figure 8 in the next section. 


\subsection{Leapfrogging, Rent-dissipation and Preemption}

In this section we consider the Riordan and Salant conjecture that was discussed in the introduction. Riordan and Salant (1994) conjectured that regardless of whether the firms move simultaneously or alternately, or whether technological progress is deterministic or stochastic, the general outcome in all of these environments is rent-dissipating preemptive investments where only one firm invests sufficiently frequently to deter its opponent from investing. These frequent preemptive investments fully dissipate any profits the investing firm can expect to earn from preempting its rival and hence also dissipate all social surplus. We first confirm their main result stated in terms of our model.

Theorem 6 (Riordan and Salant, 1994). Consider a continuous time investment game with deterministic alternating moves. Assume that the cost of investment is independent of $c, K(c)=K$ and is not prohibitively high in the sense of Assumption 1). Further, assume that technological progress is deterministic with state of the art costs at time $t \geq 0$ given by the continuous, non-decreasing function $c(t)$ and continuous time interest rate $r>0$. Assume that the continuous time analog of the condition that investment costs are not too high holds, i.e. $C(0)>r K$. Then there exists a unique MPE of the continuous time investment game (modulo relabeling of the firms) that involve preemptive investments by one or the other of the two firms and no investment in equilibrium by its opponent. The discounted payoffs of both firms in equilibrium is 0 , so the entire surplus is wasted on excessively frequent investments by the preempting firm.

Corollary 6.1 (Riordan and Salant, 1994). The continuous time equilibrium in Theorem 6 is a limit of the unique equilibria of a sequence of discrete time games where $\beta=\exp \{-r \Delta t\}$ and per period profits of the firms, $r_{i}\left(c_{1}, c_{2}\right)$, are proportional to $\Delta t$ and the order of moves alternates 
deterministically, for a deterministic sequence of state of the art costs given by $\left(c_{0}, c_{1}, c_{2}, c_{3}, \ldots\right)=$ $(c(0), c(\Delta t), c(2 \Delta t), c(3 \Delta t), \ldots)$ as $\Delta t \rightarrow 0$.

The proofs of Theorem 6 and Corollary 6.1 are given in Riordan and Salant (1994) who used a mathematical induction argument to establish the existence of the continuous time equilibrium as the limit of the equilibria of a sequence of discrete time alternating move investment games.

In Figure 8 we plot simulated MPE for three versions of the Bertrand investment pricing game with deterministic alternating moves and deterministic technological progress. In the panel (a) we let the length of the time periods be relatively small to provide a good discrete time approximation to Riordan and Salant's model in continuous time. In panel (b) we decrease the number of points of support of the marginal cost and increase the length of the time period. In panel(c) in addition we lower investment cost. These three examples demonstrate that preemptive rent-dissipating investments indeed can happen in discrete time when the cost of investing in the new technology $K(c)$ is large enough relative to per period profits, but fails when the opposite is true as shown in panels (b) and (c). In discrete time, both duopolist have temporary monopoly power that can lead to inefficient under-investment as shown in the equilibrium realization in panel (b) or leapfrogging as shown in panel (c). Since per period profits are proportional to the length of the time period, the latter increases the value of the temporary cost advantage a firm gains after investment in the state of the art technology. If investment costs are sufficiently low relative to per period profits, it can be optimal for the cost follower to leapfrog the cost leader, in the limiting case even for a one period cost leadership.

While the Riordan and Salant result of strategic preemption with full rent dissipation only 
Figure 8: Production and state of the art costs in simulated MPE: continuous vs. discrete time
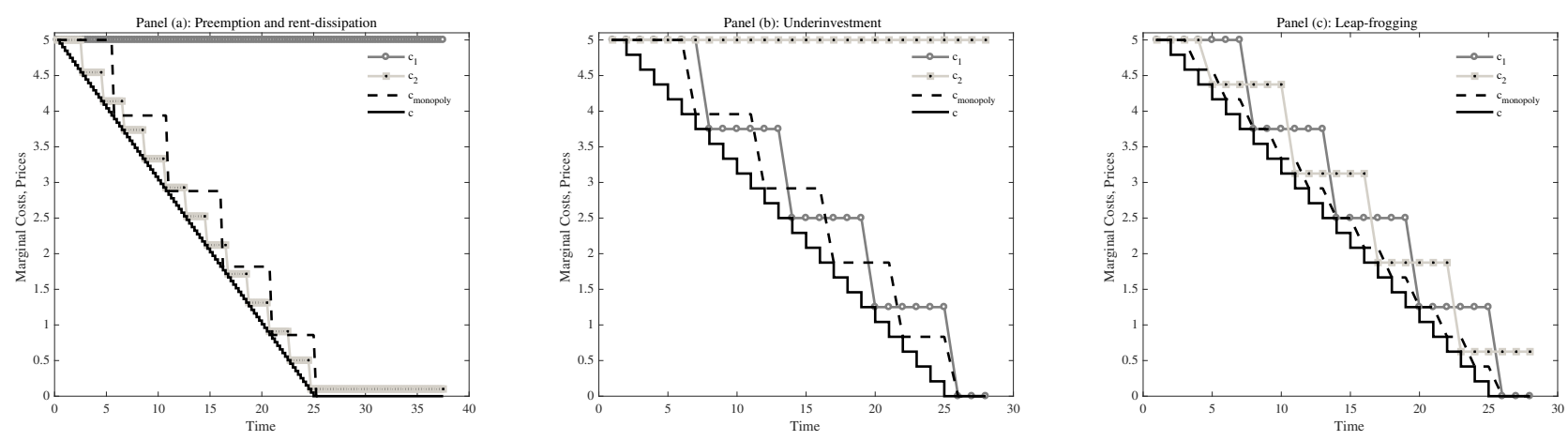

Notes: The figure plots simulated MPE equilibria for three versions of the Bertrand investment pricing game with deterministic alternating move and strictly monotonic one step technological progress. In panel (a) we present a discrete time approximation to Riordan and Salant's model, with parameters $\beta=\exp (-0.05 * \Delta t), k_{1}=2, k_{2}=0$, $\pi(c \mid c)=0, f(1 \mid 1)=f(2 \mid 2)=0, f(2 \mid 1)=f(1 \mid 2)=1, n_{c}=100$ and scale parameter for the per period profits $\Delta t=0.25$. In panel (b) we decrease the number of discrete support points of costs to $n_{c}=25$ and adjust the per period scale to $\Delta t=1$. In panel (c) in addition we lower investment costs by setting $k_{1}=0.5$.

holds in the continuous time limit $\Delta t \rightarrow 0$, their conclusion that investment preemption will occur is robust to discreteness of time. We find investment preemption is the unique equilibrium in our discrete time numerical solutions when $\Delta t$ is sufficiently small. Thus, there is a "neighborhood" of $\Delta t$ about the limit value 0 for which their unique preemption equilibrium also holds in a discrete time framework. However, the conclusion that preemption is fully inefficient and rent dissipating is not robust to discrete time. In discrete time the preempting firm does earn positive profits so the equilibrium is not completely inefficient.

Allowing for random alternation in the right to move, we obtain a unique pure strategy equilibrium, since random alternations does not violate the sufficient conditions for uniqueness given in Theorem 4. Yet, random alternation of the right to move destroys the ability to engage in strategic preemption and creates the opportunity for leapfrogging, since firms cannot have full control. Figure 9, panel (a) gives an example of a simulated equilibrium path when the right to move alternates 
Figure 9: Production and state of the art costs in simulated MPE under uncertainty
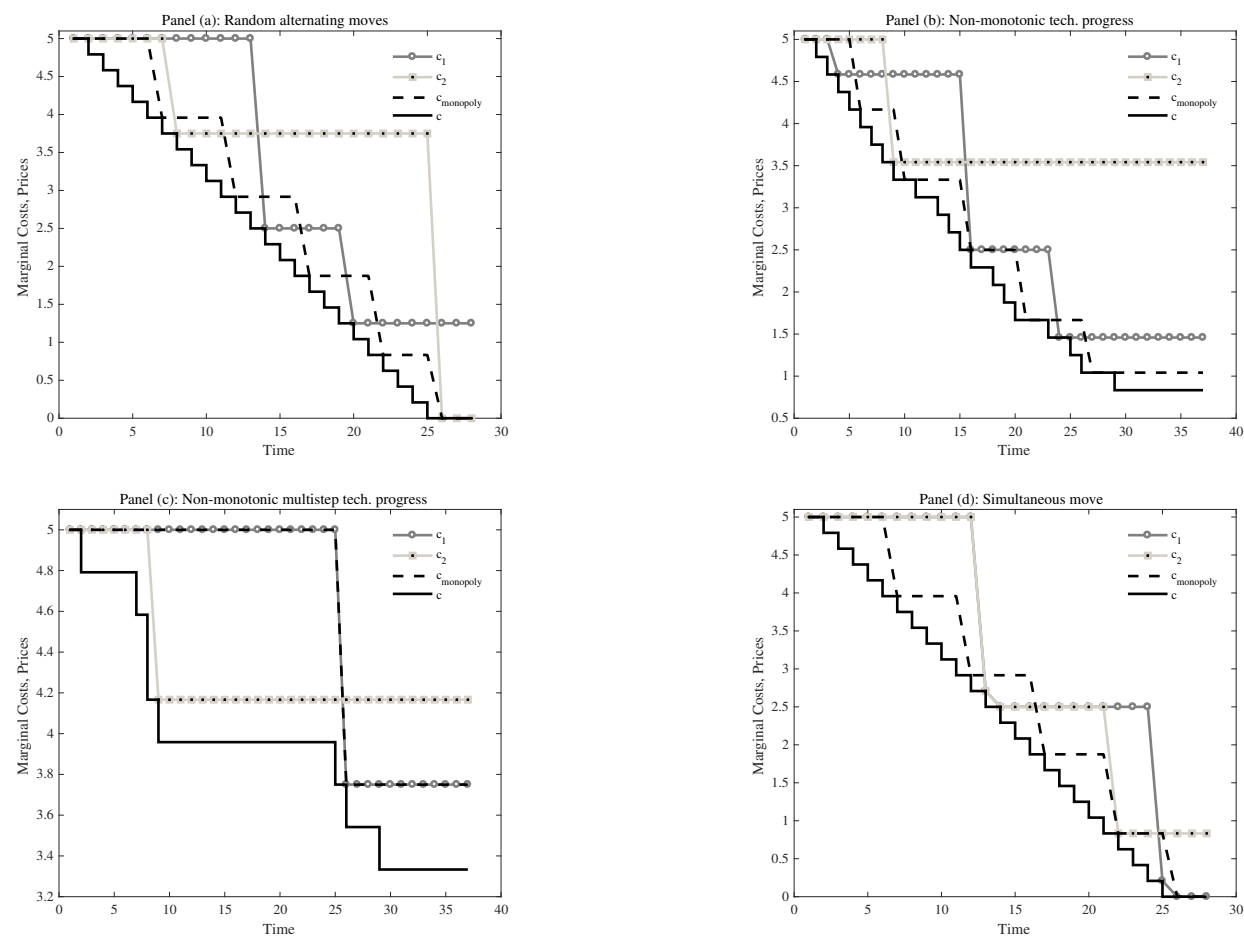

Notes: The figure plots simulated MPE by type for four stochastic generalizations of the model illustrated in panel (b) of Figure 8. In panel (a) we consider random alternating moves where $f(1 \mid 1)=f(2 \mid 2)=0.2$ and $f(2 \mid 1)=f(1 \mid 2)=$ 0.8. In panel (b) we allow for non monotonic stochastic one step technological improvement. In panel (c) we allow technological progress to follow a beta distribution with parameters $a=1.8$ and $b=0.4$, over the interval $[c, 0]$ ( $c$ is the current state-of-the-art marginal cost of production). Panel (d) plots an equilibrium path from the simultaneous move game. The remaining parameter values are as in panel (b) of Figure 8.

randomly. While this equilibrium path depicts a unique pure strategy equilibrium, we clearly see the leapfrogging pattern.

If there is positive probability of remaining with the same state of the art cost $c$ for more that one period of time, i.e. $\pi(c \mid c)>0$, the main results of Riordan and Salant (1994) will no longer hold in our model. We may have multiple equilibria, there will be leapfrogging, and full rent dissipation fails.

Figure 9 presents simulated equilibrium paths when we introduce randomness in the evolution 
of the state of the art technology, the order of moves in the alternating move game, or allow for simultaneous investments. All panels exhibit leapfrogging, reflecting the Statement that stochasticity in the model presents the cost follower with more opportunities to leapfrog its opponent and makes it harder for the cost leader to preempt leapfrogging. Overall, in presence of uncertainty, the game becomes much more contestable.

Lemma 3. (Limits to Riordan and Salant result) Preemption does not hold when (1) cost of investment $K(c)$ is sufficiently small relative to per period profits, (2) investment decisions are made simultaneously, (3) the right to move alternates randomly, (4) the probability of an advance in the state of the art technology is less than one, i.e. $\pi(c \mid c)>0$.

Proof. The proof is by counter examples which are shown in Figure 8 and 9.

The vast majority of MPE equilibria in the many specifications of the game we have solved using the RLS algorithm exhibited leapfrogging. It appears that Riordan and Salant's results are not robust to any of the mentioned assumptions, at least in the discrete time analog of their model. However, with the exception of the full rent dissipation result, we believe that there is a neighborhood about the limiting set of parameter values that Riordan and Salant used to prove Theorem 6 for which their conjectured preemption equilibrium will continue to hold, at least with high probability. 


\section{Conclusions}

The key contribution of this paper is to provide a detailed characterization of the set of equilibria of a dynamic duopoly model of Bertrand price competition in the presence of stochastic technological progress, including a characterization of the extremal equilibria of the simultaneous move version of the game, the convex hull of which includes all possible payoffs in all possible MPE. We showed this payoff set is a triangle generated from the payoffs of two asymmetric monopoly equilibria and a symmetric, zero profit mixed strategy equilibrium. The fact that the payoff set and number of possible MPE can be so large is reminiscient of the Folk Theorem for repeated games. One of the objectives of the Markov Perfect equilibrium concept was to restrict the level of "history dependence" of strategies in the hopes that it would limit the number of possible equilibria. Evidently, the Markovian restriction on strategies cannot always be relied on to achieve this goal. It is surprising (and perhaps distressing) that such a simple extension of the classic static model of Bertrand price competition, part of the foundation of industrial organization theory, can have in such a large and complex set of MPE.

Though the convex hull of the set of equilibrium payoffs shrinks and is a strict subset of triangle when the firms invest alternately rather than simultaneously (so that neither the symmetric zero profit mixed strategy payoff or either of the monopoly payoffs are no longer supportable as MPE of the alternating move game) there are still a surprisingly large number of MPE. Asynchronicity in the timing of investments prevents firms from achieving the extreme payoffs that are possible in the simultaneous move version of the game, but does not result in a unique MPE. Technology must improve with probability 1 in every period for there to be a unique MPE when firms move 
asynchronously.

Contrary to the previous literature which has focused on investment preemption as the generic equilibrium outcome, we have shown that equilibrium outcomes typically involve various types of leapfrogging that result in some of the benefits of technological progress being passed on to consumers. We have shown that these dynamic equilibria are generally inefficient due to a combination of excessively frequent investments and duplicative investments resulting from coordination failures between the firms. However, in the numerical examples we have solved the efficiency is generally very high and there even exist fully efficient asymmetric monopoly equilibria, as well as efficient non-monopoly equilibria involving perfectly coordinated leapfrogging by the two firms.

Our analysis provides an alternative interpretation of "price wars." In the equilibria of our model, price trajectories are piecewise flat with large discontinuous price drops that occur when a high cost firm leapfrogs its rival to become the new temporary low cost leader. It is via these periodic price drops that consumers benefit from technological progress and the competition between the duopolists. Unlike models of temporary sales or price wars studied in models of tacit collusion, prices never increase in this model: each price drop represents a permanent gain to consumers.

Our analysis also contributes to the long-standing debate about the relationship of market structure and innovation and the adoption of new technologies. Schumpeter (1939) argued a monopolist innovates more rapidly than a competitive industry since the monopolist can fully appropriate the benefits of R\&D or other cost-reducing investments, whereas some of these investments would be dissipated in a competitive market. However, Arrow (1962) argued that innovation (or new technology adoption) under a monopolist will be slower than in a competitive market which is in turn 
slower than the social optimum. Both types of results have appeared in the subsequent literature. For example, in the R\&D investment model analyzed by Goettler and Gordon (2011), the rate of innovation under monopoly is higher than under duopoly but still below the rate of innovation that would be chosen by a social planner. These inefficiencies are driven in part by the existence of externalities such as knowledge spillovers that are commonly associated with R\&D investments.

In a setting where each competing firm can at any time adopt an exogenously improving state of the art technology, we have shown that the rate of adoption of new cost-reducing technologies under the duopoly equilibrium is generally higher than what occurs under the monopoly or socially optimal solution. We showed that equilibria involving both leapfrogging and investment preemption both lead the duopolists to collectively invest more in cost reducing technologies than a social planner, as well as demonstrating the existence of monopoly equilibria where investments coincide with the socially optimal investment strategy. This latter result is rather specialized, and results from some of the restrictive simplifying assumptions we made to keep the analysis mathematically tractable. In particular, it would be important to extend the model to allow more than two firms and allow for entry and exit of firms. ${ }^{12}$

A disturbing aspect of our findings from the standpoint of "taking models to data" is the plethora of Markov perfect equilibria even in a such a simple extension of the classic static model of Bertrand price competition. Though we have shown that the number of MPE shrinks dramatically under an alternating move specification of the game and a unique MPE obtains when the

\footnotetext{
${ }^{12}$ We refer readers to the original work by Reinganum (1985) as well as recent work by Acemoglu and Cao (2015) and the large literature they build on. It is an example of promising new models of endogenous innovation by incumbents and new entrants. In their model entrants are responsible for more "drastic" innovations that tend to replace incumbents, who focus on less drastic innovations that improve their existing products.
} 
probability of technological improvement in every time period is sufficiently close to one, there will generally be a huge multiplicity of equilibria either when firms move simultaneously, or when the probability of technological improvement is sufficiently low. Thus, though leapfrogging can be viewed as an endogenous solution to the "anti-coordination problem" and provides a resolution

to the "Bertrand investment paradox" our paper leaves unsolved the more general question of how firms coordinate on a single equilibrium when there is a vast multiplicity of them, or if Markov Perfect Equilibrium provides a good approximation to how firms actually behave. These questions, together with the computational difficulties of computing and selecting an "empirically relevant" equilibrium out of a vast multiplicity of possible equilibria pose major challenges to the application of Markov Perfect Equilibrium in empirical industrial organization, such as the nascent literature inspired by Ericson and Pakes (1995).

\section{References}

[1] Acemoglu, Daron, and Dan Cao (2015) "Innovation by entrants and incumbents" Journal of Economic Theory 157 255-294.

[2] Arrow, K.J. (1962) "Economic Welfare and the Allocation of Resources for Inventions" in Nelson, R.R. (ed.), The Rate and Direction of Inventive Activity: Economic and Social Factors Princeton University Press. Princeton.

[3] Baumol, W.J. and Panzar, J.C. and Willig, R.D (1982) Contestable Markets and the Theory of Industry Structure Harcourt, Brace Jovanovich.

[4] Bertrand, J. (1883) [Review of] "Théorie Mathématique de la Richesse Social par Léon Walras: Recherches sur les Principes de la Théorie du Richesses par Augustin Cournot" Journal des Savants 67 499-508.

[5] Ericson, R. and A. Pakes (1995) "Markov Perfect Industry Dynamics: A Framework for Empirical Work" Review of Economic Studies 62-1 58-82.

[6] Fudenberg, D. R. Gilbert, J Stiglitz and J. Tirole (1983) "Preemption, leapfrogging and competition in patent races" European Economic Review 22-1 3-31. 
[7] Gilbert, R and D. Newbery (1982) "Pre-emptive Patenting and the Persistence of Monopoly" American Economic Review 74 514-526.

[8] Giovannetti, Emanuelle (2001) "Perpetual Leapfrogging in Bertrand Duopoly" International Economic Review 42-3 671-696.

[9] Goettler, Ronald and Brett Gordon (2011) “Does AMD spur Intel to innovate more?” Journal of Political Economy 119-6 1141-2000.

[10] Iskhakov, F., Rust, J. and B. Schjerning (2016) "Recursive Lexicographical Search: Finding all Markov Perfect Equilibria of Finite State Directional Dynamic Games" Review of Economic Studies 83 658-703.

[11] Lagunoff, R. and A. Matsui (1997) “Asynchronous Choice in Repeated Coordination Games" Econometrica 65-6 1467-1477.

[12] Reinganum, J. (1985) "Innovation and Industry Evolution" Quarterly Journal of Economics 100-1 81-99.

[13] Ried, W. (1993) "Existence and Structure of Nash Equilibria in Simple Asymmetric Models of Bertrand Price Competition” Jahrbuch für Sozialwissenschaft Bd. 44, H. 2 216-232.

[14] Riordan, M. and D. Salant (1994) "Preemptive Adoptions of an Emerging Technology" Journal of Industrial Economics 42-3 247-261.

[15] Routledge, Robert R. (2010) "Bertrand competition with cost uncertainty" Economics Letters $107356-359$.

[16] Schumpeter, J.A. (1939) Business Cycles: A Theoretical, Historical and Statistical Analysis of the Capitalist Process. New York: McGraw Hill.

[17] Vickers. M. (1986) "The Evolution of Market Structure When There is a Sequence of Innovations" Journal of Industrial Economics 35 1-12. 


\section{A Proofs of Lemmas and Theorems}

Theorem 1 (Necessary and sufficient condition for investment by the social planner).

Proof. Note that the left hand side of inequality (13) is the discounted cost savings from adopting the state of the art technology $c^{\prime}$ when the existing plant has marginal cost $c_{1}$. We first prove that if this inequality holds, then investment will be socially optimal at some state $\left(c_{1}{ }^{\prime}, c^{\prime}\right)$ satisfying $c_{1}^{\prime} \in\left[0, c_{1}\right]$ and $c^{\prime} \in\left[0, c_{1}^{\prime}\right]$. Suppose, to the contrary, that investment is not optimal for the social planner for any value $c_{1}^{\prime} \leq c_{1}$ and any $c^{\prime} \in\left[0, c_{1}^{\prime}\right]$. It follows that $C\left(c_{1}^{\prime}, c^{\prime}\right)=c_{1}^{\prime} /(1-\beta)$ for all $c_{1}^{\prime} \leq c_{1}$ and all $c^{\prime} \in\left[0, c_{1}^{\prime}\right]$. However if the social planner did decide to invest when the state is $\left(c_{1}, c\right)$ the planner's discounted costs would be $c_{1}+K(c)+\beta \int_{0}^{c} C\left(c, c^{\prime}\right) \pi\left(d c^{\prime} \mid c\right)$. Since we have assumed it is not optimal for the social planner to invest at any state $\left(c_{1}^{\prime}, c^{\prime}\right)$ with $c^{\prime} \in\left[0, c_{1}^{\prime}\right]$, then it cannot be optimal to invest in particular at any state $\left(c_{1}, c\right)$ with $c \in\left[0, c_{1}\right]$. It follows that $c_{1} /(1-\beta) \leq c_{1}+K(c)+\beta \int_{0}^{c} C\left(c, c^{\prime}\right) \pi\left(d c^{\prime} \mid c\right)$ for all $c \in\left[0, c_{1}\right]$. However since $C\left(c, c^{\prime}\right)=$ $c /(1-\beta)$ for all $c^{\prime} \in[0, c]$, it follows that $\beta c_{1} /(1-\beta) \leq K(c)+\beta c /(1-\beta)$ for all $c \in\left[0, c_{1}\right]$, but this contradicts inequality (13).

Conversely, suppose inequality (13) does not hold. Then it follows that there is no value of $c^{\prime} \in\left[0, c_{1}\right]$ for which investment is optimal, since in this case $C\left(c_{1}, c^{\prime}\right)=c_{1} /(1-\beta)$ for all $c^{\prime} \in$ $\left[0, c_{1}\right]$. This latter result follows by verifying that it is a solution to the Bellman equation (11), where it follows that the cost of replacing a plant with marginal cost $c^{\prime}$ when the state of the art marginal cost is $c^{\prime}$ is $c_{1}+K\left(c^{\prime}\right)+\beta c^{\prime} /(1-\beta)$ which exceeds the cost of keeping the existing plant $C\left(c_{1}, c^{\prime}\right)=c_{1} /(1-\beta)$ by our assumption that inequality (13) does not hold for any $c^{\prime} \in\left[0, c_{1}\right]$. Since the solution to the Bellman equation is unique (via the contraction mapping property) and corresponds to an optimal investment policy, we conclude that there is no state $\left(c_{1}, c^{\prime}\right)$ with $c^{\prime} \in$ $\left[0, c_{1}\right]$ for which investment in the state of the art technology $c^{\prime}$ is socially optimal.

Before proving the main results (Theorems 2 and 3) we preset a number of lemmas needed for these proofs.

Lemma A.1 (Necessary condition for social optimality of no investment at a particular state $\left.\left(c_{1}, c\right)\right)$. Suppose that it is not optimal for the social planner (or monopolist) to invest at state $\left(c_{1}, c\right), c_{1} \geq c$. It must then hold

$$
K(c)>\beta E\left\{\sum_{t=1}^{\tilde{\tau}-1} \beta^{t-1}\left(c_{1}-c\right)\right\}=\frac{\left(c_{1}-c\right)\left(\beta-E\left\{\beta^{\tilde{\tau}}\right\}\right)}{1-\beta}
$$

where $\tilde{\tau}$ denotes the random number of time periods when investment will remain socially nonoptimal.

Proof. Essentially, the Lemma A.1 states that whenever the social planner is optimally choosing not to invest at state $\left(c_{1}, c\right)$, it must be that the expected discounted saving in production cost does not compensate for the cost of investment. From the point of view of the current time period, the potential saving in production cost is given by the difference in cost of production over $\tilde{\tau}$ periods starting from the following one, and up to and including the period when investment is 
again optimal. In other words, it is given by the partial sum of the geometric series with the constant element equal to $c_{1}-c$ and the factor $\beta<1$ taken over $\tilde{\tau}-1$ periods, which also has to be discounted once more because of the one period time to build. After $\tilde{\tau}$ periods the state of the art cost $c$ will reach the lower level $\tilde{c}_{\tilde{\tau}}<c$ and the investment by the social planner will again be justified, unless $\tilde{\tau}=\infty$ in which case (22) simplifies to (13), and by Theorem 1 no more investment ever occurs.

When investment is not optimal at $\left(c_{1}, c\right)$, it follows from the social planner Bellman equation (11) that

$$
C\left(c_{1}, c\right)=c_{1}+\beta \int_{0}^{c} C\left(c_{1}, c^{\prime}\right) \pi\left(d c^{\prime} \mid c\right)
$$

and from (12) it follows that

$$
K(c)>\beta \int_{0}^{c}\left[C\left(c_{1}, c^{\prime}\right)-C\left(c, c^{\prime}\right)\right] \pi\left(d c^{\prime} \mid c\right) .
$$

It is also trivial to show that the investment at $(c, c)$ is not optimal as it only entails to wasting the investment cost, giving

$$
C(c, c)=c+\beta \int_{0}^{c} C\left(c, c^{\prime}\right) \pi\left(d c^{\prime} \mid c\right) .
$$

Combining (23), (24) and (25) we get

$$
K(c)>\left[C\left(c_{1}, c\right)-C(c, c)\right]-\left[c_{1}-c\right] .
$$

Consider then a particular investment policy starting in state $(c, c)$, not investing for $\tilde{\tau}$ periods, then investing in the state of the art technology $\tilde{c}_{\tilde{\tau}}$ in the next period, and then following the socially optimal investment policy thereafter. Denote the cost associated with this strategy by $C_{\tilde{\tau}}(c, c)$. Because $C(c, c)$ is the minimal cost under an optimal investment policy, it follows that $C(c, c) \leq$ $C_{\tilde{\tau}}(c, c)$, and from (26) we have

$$
K(c)>\left[C_{1}\left(c_{1}, c\right)-C_{\tilde{\tau}}(c, c)\right]-\left[c_{1}-c\right]
$$

Since $C_{\tilde{\tau}}(c, c)$ is the discounted expected cost of following, with probability 1, the same optimal investment policy that the social planner would follow once the state of the art cost reduces to $\tilde{\tilde{\tau}} \tilde{\tau}$, it follows that

$$
C\left(c_{1}, c\right)-C_{\tilde{\tau}}(c, c)=E\left\{\sum_{t=1}^{\tilde{\tau}} \beta^{t-1}\left(c_{1}-c\right)\right\}=\frac{\left(c_{1}-c\right)\left(1-E\left\{\beta^{\tilde{\tau}}\right\}\right)}{1-\beta},
$$

i.e. the difference in the values is simply the total expected discounted difference in per period costs, $c_{1}-c$, from not investing for $\tilde{\tau}$ periods when initial production costs are $c_{1}$ and $c$, respectively, and following the optimal investment policy from the point $\left(c_{1}, \tilde{c \tau}\right)$ thereafter. Substituting (28) into (27) we obtain inequality (22). 
Lemma A.2 (Necessary condition for no investment equilibrium in the whole Bertrand pricing and investment game). In any Markov perfect equilibrium of the simultaneous and alternative move formulation of the Bertrand investment game where neither firm invests, we must have

$$
K(c) \geq \frac{\beta\left(\min \left(c_{1}, c_{2}\right)-c\right)}{1-\beta}
$$

in every state $\left(c_{1}, c_{2}, c\right) \in S=\left\{\left(c_{1}, c_{2}, c\right): c \leq c_{1} \leq c_{0}, c \leq c_{2} \leq c_{0}, 0 \leq c \leq c_{0}\right\}$. That is, there is no state of the art cost $c$ for which a social planner would find it optimal to invest either.

Proof. Consider the simultaneous move game first, i.e. the case $m=0$ (omitted below for clarity of exposition). Assume that $c_{1} \leq c_{2}$. This is without loss of generality since if $c_{1}>c_{2}$ we can repeat the argument below from firm 2's perspective. If it is an equilibrium for neither firm to invest, it follows that in any state $\left(c_{1}, c_{2}, c\right)$ satisfying $c_{1} \leq c_{2}$ we have

$$
\begin{aligned}
v_{N, 1}\left(c_{1}, c_{2}, c\right) & =\frac{c_{2}-c_{1}}{1-\beta}, \\
v_{I, 1}\left(c_{1}, c_{2}, c\right) & =c_{2}-c_{1}-K(c)+\frac{\beta\left(c_{2}-c\right)}{1-\beta},
\end{aligned}
$$

and that $v_{N, 1}\left(c_{1}, c_{2}, c\right) \geq v_{I, 1}\left(c_{1}, c_{2}, c\right)$. This implies that the following inequality must hold $\forall c_{1} \in$ $\left[0, c_{2}\right]$ and $\forall c \in\left[0, c_{1}\right]$

$$
\frac{c_{2}-c_{1}}{1-\beta} \geq\left(c_{2}-c_{1}\right)-K(c)+\beta \frac{\left(c_{2}-c\right)}{1-\beta} .
$$

A similar inequality exist for $\forall c_{2} \in\left[0, c_{1}\right]$ and $\forall c \in\left[0, c_{2}\right]$. It is easy to see via simple algebra that these inequalities are equivalent to inequality (29).

Now consider the alternating move game, $m \neq 0$. Recall that $c_{0}$ is the initial value of the state of the marginal production $c$. It is not hard to show, using the Bellman equations for the alternating move game (see equation 7 in section 2), that if it is never optimal for either firm to invest, then it follows that for the state $\left(c_{1}, c_{2}, c\right)=\left(c_{0}, c_{2}, c\right)$, that $P_{2}\left(c_{0}, c_{2}, c\right)=0$ for all $c_{2} \in\left[0, c_{0}\right]$ and for all $c \in\left[0, c_{1}\right]$. But this will be true if and only if

$$
\begin{aligned}
v_{N, 1}\left(c_{1}, c_{2}, c, 1\right) & =\frac{c_{2}-c_{1}}{1-\beta} \\
v_{I, 1}\left(c_{1}, c_{2}, c, 1\right) & =c_{2}-c_{1}-K(c)+\frac{\beta\left(c_{2}-c\right)}{1-\beta}
\end{aligned}
$$

and $v_{N, 1}\left(c_{1}, c_{2}, c, 1\right) \geq v_{I, 1}\left(c_{1}, c_{2}, c, 1\right)$. It is easy to see that this is equivalent to inequality (30) above and that a similar inequality can be derived from the perspective of firm 2 . In turn inequality (29) must hold, thereby proving Lemma A.2.

Lemma A.3 (Stage game equilibria in the simultaneous move game when $c=0$ ). Consider the stage games of the simultaneous move game $(m=0)$ where $c=0$. In states where investment is not socially optimal in the sense of Assumption 1, the investment game has a unique pure strategy equilibrium where neither firm invests. When investment is socially optimal, the investment game has three subgame perfect Nash equilibria: two pure strategy "anti-coordination" equilibria and one mixed strategy equilibrium. 
Proof. When $c=0$, it follows trivially from Theorem 1 that the social planner operating the minimum cost plant will invest in the state of the art technology if and only if

$$
\frac{\beta \min \left(c_{1}, c_{2}\right)}{1-\beta}>K(0)
$$

Now consider the Nash equilibria of the $\left(c_{1}, c_{2}, 0\right)$ end game. It follows from A.2 that unless (31) holds, i.e investment is optimal for the social planner, then there is only a single "no investment" equilibrium.

Consider the states where $c_{1}=0$ or $c_{2}=0$ where one or both firms had already invested in the state of the art technology $c=0$. Condition (31) does not hold in these states, and therefore the only MPE is no investment by either firm. It then follows from (6) for firm 1 that

$$
\begin{array}{rcc}
v_{N, 1}(0,0,0)=0, & v_{I, 1}(0,0,0)=-K(0), & P_{1}(0,0,0)=0, \\
v_{N, 1}\left(c_{1}, 0,0\right)=0, & v_{I, 1}\left(c_{1}, 0,0\right)=-K(0), & P_{1}\left(c_{1}, 0,0\right)=0 \\
v_{N, 1}\left(0, c_{2}, 0\right)=\frac{c_{2}}{1-\beta}, & v_{I, 1}\left(0, c_{2}, 0\right)=\frac{c_{2}}{1-\beta}-K(0), & P_{1}\left(0, c_{2}, 0\right)=0
\end{array}
$$

where $c_{1}>0$ and $c_{2}>0$. Similar expressions hold for firm 2:

$$
\begin{array}{rcc}
v_{N, 2}(0,0,0)=0, & v_{I, 2}(0,0,0)=-K(0), & P_{2}(0,0,0)=0, \\
v_{N, 2}\left(c_{1}, 0,0\right)=\frac{c_{1}}{1-\beta}, & v_{I, 2}\left(c_{1}, 0,0\right)=\frac{c_{1}}{1-\beta}-K(0), & P_{2}\left(c_{1}, 0,0\right)=0, \\
v_{N, 2}\left(0, c_{2}, 0\right)=0, & v_{I, 2}\left(0, c_{2}, 0\right)=-K(0), & P_{2}\left(0, c_{2}, 0\right)=0 .
\end{array}
$$

Now consider the states $\left\{\left(c_{1}, c_{2}, 0\right): c_{1}>0, c_{2}>0\right\}$ in the case when investment is socially optimal, i.e. condition (31) holds. ${ }^{13}$ Focusing on firm 1 again, the Bellman equations (6) simplify to

$$
\begin{aligned}
v_{N, 1}\left(c_{1}, c_{2}, 0\right) & =r_{1}\left(c_{1}, c_{2}\right)+\beta\left(1-P_{2}\left(c_{1}, c_{2}, 0\right)\right) \max \left\{v_{N, 1}\left(c_{1}, c_{2}, 0\right), v_{I, 1}\left(c_{1}, c_{2}, 0\right)\right\} \\
v_{I, 1}\left(c_{1}, c_{2}, 0\right) & =r_{1}\left(c_{1}, c_{2}\right)-K(0)+\beta\left(1-P_{2}\left(c_{1}, c_{2}, 0\right)\right) \frac{c_{2}}{1-\beta} .
\end{aligned}
$$

Consider first the pure strategy equilibria at these states. If firm 2 invests with probability $P_{2}\left(c_{1}, c_{2}, 0\right)=1$, then the last terms in (34) disappear, leading to $v_{N, 1}\left(c_{1}, c_{2}, 0\right)>v_{I, 1}\left(c_{1}, c_{2}, 0\right)$. Thus firm 1 does not invest, i.e. $P_{1}\left(c_{1}, c_{2}, 0\right)=0$, forming the first pure strategy "anti-coordination" equilibrium. Conversely, if firm 2 surely does not invest, i.e. $P_{2}\left(c_{1}, c_{2}, 0\right)=0$, there are two possibilities. If $v_{N, 1}\left(c_{1}, c_{2}, 0\right)<v_{I, 1}\left(c_{1}, c_{2}, 0\right)$ and thus $P_{1}\left(c_{1}, c_{2}, 0\right)=1$, together with (34) this leads to the same inequality as condition (31). This establishes the second pure strategy "anti-coordination" equilibrium where firm 1 invests and firm 2 doesn't. If, on the other hand $v_{N, 1}\left(c_{1}, c_{2}, 0\right)>v_{I, 1}\left(c_{1}, c_{2}, 0\right)$ we get a contradiction. Similar argument establishes the symmetric result for firm 2 .

\footnotetext{
${ }^{13}$ The rest of the proof follows closely the proof of Theorem 6 in Iskhakov, Rust, Schjerning (2016).
} 
Consider now the mixed strategy equilibrium in the states $\left\{\left(c_{1}, c_{2}, 0\right): c_{1}>0, c_{2}>0\right\}$, assuming that (31) holds. For some level of $P_{2}\left(c_{1}, c_{2}, 0\right)$ firm 1 can be indifferent between investing and not investing, in which case any $P_{1}\left(c_{1}, c_{2}, 0\right) \in[0,1]$ would constitute its best response investment probability. In this case combining (34) with the equality $v_{N, 1}\left(c_{1}, c_{2}, 0\right)=v_{I, 1}\left(c_{1}, c_{2}, 0\right)$ gives rise to the following quadratic equation in $P_{2}=P_{2}\left(c_{1}, c_{2}, 0\right)$

$$
Q_{1}\left(P_{2}\right)=\frac{\beta^{2} c_{2}}{1-\beta}\left(1-P_{2}\right)^{2}+\beta\left(r_{1}\left(c_{1}, c_{2}\right)-K(0)-\frac{c_{2}}{1-\beta}\right)\left(1-P_{2}\right)+K(0)=0 .
$$

Conditional on falling to the unit interval, the roots of (35) paired with the roots of the symmetric equation for firm 2 would define the mixed strategy equilibrium of the stage game. Note however, that for $P_{2}=1$ the left hand side of (35) is equal to $K(0)>0$, while for $P_{2}=0$ it is equal to

$$
(1-\beta)\left(K(0)-\frac{\beta\left(c_{2}-r_{1}\left(c_{1}, c_{2}\right)\right)}{1-\beta}\right)=(1-\beta)\left(K(0)-\frac{\left.\beta \min \left(c_{1}, c_{2}\right)\right)}{1-\beta}\right)<0,
$$

and thus only a single root lies in the unit interval. Similar argument holds for firm 2. This establishes the existence and uniqueness of the mixed strategy equilibrium, and concludes the proof of the lemma.

Lemma A.4 (Leapfrogging in the simultaneous move game when $c=0$ ). In the mixed strategy equilibria in Lemma A.3 the high cost firm has the higher probability of investing, i.e. $P_{1}\left(c_{1}, c_{2}, 0\right)>P_{2}\left(c_{1}, c_{2}, 0\right)$ when $c_{1}>c_{2}$, and $P_{1}\left(c_{1}, c_{2}, 0\right)<P_{2}\left(c_{1}, c_{2}, 0\right)$ when $c_{1}<c_{2}$.

Proof. The proof builds on Lemma A.3 where it shows the existence of a single mixed strategy equilibrium in the states $\left\{\left(c_{1}, c_{2}, 0\right): c_{1}>0, c_{2}>0\right\}$ under condition (31). The only root of the equation (35) that lies in the unit interval defines the equilibrium probability of investment of firm $2, P_{2}\left(c_{1}, c_{2}, 0\right)$. The symmetric equation that defines the equilibrium investment probability $P_{1}=P_{1}\left(c_{1}, c_{2}, 0\right)$ of firm 1 is

$$
Q_{2}\left(P_{1}\right)=\frac{\beta^{2} c_{1}}{1-\beta}\left(1-P_{1}\right)^{2}+\beta\left(r_{2}\left(c_{1}, c_{2}\right)-K(0)-\frac{c_{1}}{1-\beta}\right)\left(1-P_{1}\right)+K(0)=0 .
$$

Evaluating the left hand sides of equations (35) and (36) at the ends of the unit interval we have

$$
\begin{aligned}
& Q_{1}(0)=Q_{2}(0)=(1-\beta)\left(K(0)-\frac{\left.\beta \min \left(c_{1}, c_{2}\right)\right)}{1-\beta}\right)<0, \\
& Q_{1}(1)=Q_{2}(1)=K(0)>0 .
\end{aligned}
$$

Because both $Q_{1}\left(P_{2}\right)$ and $Q_{2}\left(P_{1}\right)$ are quadratic polynomials and have the same values at 0 and 1 , it is sufficient to compare their derivatives on one side of the unit interval to establish the order of their roots on this interval. We have

$$
\left.\frac{\partial Q_{1}\left(P_{2}\right)}{\partial P_{2}}\right|_{P_{2}=1}-\left.\frac{\partial Q_{2}\left(P_{1}\right)}{\partial P_{1}}\right|_{P_{1}=1}=\beta\left[r_{2}\left(c_{1}, c_{2}\right)-r_{1}\left(c_{1}, c_{2}\right)+\frac{c_{2}-c_{1}}{1-\beta}\right]
$$


For the case $c_{1}<c_{2}$ the difference in (37) evaluates to $\frac{\beta^{2}\left(c_{2}-c_{1}\right)}{1-\beta}>0$ which implies that the root of $Q_{1}\left(P_{2}\right)$ in the unit interval lies to the right of the root of $Q_{2}\left(P_{1}\right)$. In other words, $P_{2}\left(c_{1}, c_{2}, 0\right)>$ $P_{1}\left(c_{1}, c_{2}, 0\right)$ when $c_{1}<c_{2}$. Conversely, for the case $c_{1}>c_{2}$ the difference in (37) evaluates to $-\frac{\beta^{2}\left(c_{1}-c_{2}\right)}{1-\beta}>0$ which implies that the root of $Q_{1}\left(P_{2}\right)$ in the unit interval lies to the left of the root of $Q_{2}\left(P_{1}\right)$. In other words, $P_{2}\left(c_{1}, c_{2}, 0\right)<P_{1}\left(c_{1}, c_{2}, 0\right)$ when $c_{1}>c_{2}$. We conclude that in the state of the game $\left\{\left(c_{1}, c_{2}, 0\right): c_{1}>0, c_{2}>0\right\}$ where the mixed strategy equilibrium exists under condition (31), the firm with higher marginal cost of production has a higher probability of investment.

Lemma A.5 (Efficiency of the stage game equilibria in the simultaneous move game when $c=0$ ). The pure strategy equilibria in Lemma A.3 are fully efficient. The mixed strategy equilibria in Lemma A.3 are inefficient due to the possibility of over-or underinvestment.

Proof. If follows from Lemma A.3 that in pure strategy equilibria investment by any firm takes place if and only if the condition (31) is satisfied, i.e. it is socially optimal to invest. Moreover, because in these "anti-coordination" equilibria no more than firm invests at a time, there is no possibility for efficiency loss due to duplicative investment. Thus, the pure strategy equilibria at the states $\left(c_{1}, c_{2}, 0\right)$ are fully efficient.

Consider then the mixed strategy equilibria in states $\left\{\left(c_{1}, c_{2}, 0\right): c_{1}>0, c_{2}>0\right\}$ where it is socially optimal to invest, i.e. assuming (31) is satisfied. Let $C_{m}\left(c_{1}, c_{2}, 0\right)$ denote the present discounted value of investment and production costs under this mixed strategy equilibrium at the point $\left(c_{1}, c_{2}, 0\right)$. Suppressing the arguments $\left(c_{1}, c_{2}, 0\right)$ of the investment probabilities, we have

$$
\begin{aligned}
C_{m}\left(c_{1}, c_{2}, 0\right)= & \min \left(c_{1}, c_{2}\right)+2 K(0) P_{1} P_{2}+K(0) P_{1}\left(1-P_{2}\right)+K(0)\left(1-P_{1}\right) P_{2} \\
& +\beta\left(1-P_{1}\right)\left(1-P_{2}\right) C_{m}\left(c_{1}, c_{2}, 0\right) \\
= & \min \left(c_{1}, c_{2}\right)+K(0)\left(P_{1}+P_{2}\right)+\beta\left(1-P_{1}\right)\left(1-P_{2}\right) C_{m}\left(c_{1}, c_{2}, 0\right) .
\end{aligned}
$$

We will now show that $C_{m}\left(c_{1}, c_{2}, 0\right)=\left(K(0)\left(P_{1}+P_{2}\right)+c_{1}\right) /\left(1-\beta\left(1-P_{1}\right)\left(1-P_{2}\right)\right)$ exceeds the socially optimal production and investment costs $C^{*}\left(c_{1}, c_{2}, 0\right)=\min \left(c_{1}, c_{2}\right)+K(0)$ that a social planner can achieve by undertaking only a single investment in the state of the art technology and avoid i) the higher costs due to redundant duplicative investments and ii) the costs due to delayed investment due to the probability $\left(1-P_{1}\right)\left(1-P_{2}\right)$ that neither firm invests under the mixed strategy equilibrium.

We establish this inequality via an indirect argument. Let $p=P_{1}+P_{2}-P_{1} P_{2} \in(0,1)$ be the probability that at least one of the firms invests in the mixed strategy equilibrium, and let $\underline{C}_{m}(p)<C_{m}\left(c_{1}, c_{2}, 0\right)$ denote the present value of costs under a mixed strategy equilibrium that ignores the occurrence of redundant investments by the two firms and therefore constitutes the lower bound on $C_{m}\left(c_{1}, c_{2}, 0\right)$. We write $\underline{C}_{m}(p)$ to emphasize its dependence on $p$.

$$
\begin{aligned}
\underline{C}_{m}(p) & =\min \left(c_{1}, c_{2}\right)+p K(0)+\beta(1-p) \underline{C}_{m}(p) \\
& =\frac{\min \left(c_{1}, c_{2}\right)+p K(0)}{1-\beta(1-p)} .
\end{aligned}
$$


Note that $\underline{C}_{m}(1)$ equals the social optimal level of cost, $C^{*}\left(c_{1}, c_{2}, 0\right)$. It then suffices to show that $\underline{C}_{m}(p)$ is a decreasing function:

$$
\frac{d \underline{C}_{m}(p)}{d p}=\frac{K(0)(1-\beta)-\beta \min \left(c_{1}, c_{2}\right)}{(1-\beta(1-p))^{2}}<0,
$$

where the inequality holds under (31). Since we know that $p \in(0,1)$ in the mixed strategy equilibrium, we have

$$
C_{m}\left(c_{1}, c_{2}, 0\right)>\underline{C}_{m}(p)>C^{*}\left(c_{1}, c_{2}, 0\right) .
$$

which establishes the inefficiency of the mixed strategy equilibrium.

Theorem 2 (The set of equilibrium payoffs in the Simultaneous Move Game).

We prove Theorem 2 statement by statement. It is helpful to re-write the Bellman equations (6) as

$$
\begin{aligned}
v_{N, 1}\left(c_{1}, c_{2}, c\right) & =r_{1}\left(c_{1}, c_{2}\right)+\beta\left[P_{2}\left(c_{1}, c_{2}, c\right) H_{1}\left(c_{1}, c, c\right)+\left(1-P_{2}\left(c_{1}, c_{2}, c\right)\right) H_{1}\left(c_{1}, c_{2}, c\right)\right], \quad(40) \\
v_{I, 1}\left(c_{1}, c_{2}, c\right) & =r_{1}\left(c_{1}, c_{2}\right)-K(c)+\beta\left[P_{2}\left(c_{1}, c_{2}, c\right) H_{1}(c, c, c)+\left(1-P_{2}\left(c_{1}, c_{2}, c\right)\right) H_{1}\left(c, c_{2}, c\right)\right]
\end{aligned}
$$

where the expected value function $H_{1}\left(c_{1}, c_{2}, c\right)$ is given by

$$
\begin{aligned}
H_{1}\left(c_{1}, c_{2}, c\right) & =h_{1}\left(c_{1}, c_{2}, c\right)+\pi(c \mid c) \max \left\{v_{N, 1}\left(c_{1}, c_{2}, c\right), v_{I, 1}\left(c_{1}, c_{2}, c\right)\right\} \\
h_{1}\left(c_{1}, c_{2}, c\right) & =(1-\pi(c \mid c)) \int_{0}^{c} \max \left\{v_{N, 1}\left(c_{1}, c_{2}, c^{\prime}\right), v_{I, 1}\left(c_{1}, c_{2}, c^{\prime}\right)\right\} p\left(d c^{\prime} \mid c\right)
\end{aligned}
$$

Here $\pi(c \mid c)$ denotes the probability for a cost-reducing innovation not to occur, and $p\left(c^{\prime} \mid c\right)$ is the conditional density of the new (lower) state of the art marginal cost of production conditional on an innovation having occurred, since Assumption 2 implies that $\pi\left(c^{\prime} \mid c\right)$ has the representation

$$
\pi\left(c^{\prime} \mid c\right)= \begin{cases}{[1-\pi(c \mid c)] \int_{0}^{c^{\prime}} p(\hat{c} \mid c) d \hat{c}} & c^{\prime}<c \\ 1 & c^{\prime} \geq c .\end{cases}
$$

The Bellman equations for firm 2 have the symmetric form

$$
\begin{aligned}
v_{N, 2}\left(c_{1}, c_{2}, c\right) & =r_{2}\left(c_{1}, c_{2}\right)+\beta\left[P_{1}\left(c_{1}, c_{2}, c\right) H_{2}\left(c, c_{2}, c\right)+\left(1-P_{1}\left(c_{1}, c_{2}, c\right)\right) H_{2}\left(c_{1}, c_{2}, c\right)\right], \quad \text { (44) } \\
v_{I, 2}\left(c_{1}, c_{2}, c\right) & =r_{2}\left(c_{1}, c_{2}\right)-K(c)+\beta\left[P_{1}\left(c_{1}, c_{2}, c\right) H_{2}(c, c, c)+\left(1-P_{1}\left(c_{1}, c_{2}, c\right)\right) H_{2}\left(c_{1}, c, c\right)\right],
\end{aligned}
$$

where the expected value function $H_{2}\left(c_{1}, c_{2}, c\right)$ is given by

$$
\begin{aligned}
H_{2}\left(c_{1}, c_{2}, c\right) & =h_{2}\left(c_{1}, c_{2}, c\right)+\pi(c \mid c) \max \left\{v_{N, 2}\left(c_{1}, c_{2}, c\right), v_{I, 2}\left(c_{1}, c_{2}, c\right)\right\} \\
h_{2}\left(c_{1}, c_{2}, c\right) & =(1-\pi(c \mid c)) \int_{0}^{c} \max \left\{v_{N, 2}\left(c_{1}, c_{2}, c^{\prime}\right), v_{I, 2}\left(c_{1}, c_{2}, c^{\prime}\right)\right\} p\left(d c^{\prime} \mid c\right)
\end{aligned}
$$


Statement 1. (No investments by both firms in all states $\left(c_{1}, c_{2}, c\right)$ is not supported in any of the MPE equilibria of the game).

Proof. By Theorem 1, if investment is optimal for the social planner, then inequality (29) cannot hold. By Lemma A.2, it follows that no investment cannot be an MPE outcome.

Statement 2. (There are two fully efficient "monopoly" equilibria in which either one or the other firm makes all the investments and earns maximum feasible profit while their opponent earns zero profits).

Proof. The two candidate equilibria are where firm 2 never invests in equilibrium and firm 1 does all the investing (whenever it is profit-maximizing for firm 1 to do so), and symmetrically, where firm 1 never invests and firm 2 does all the investing (whenever it is profit-maximizing for firm 2 to do so). Without loss of generality, we only consider the case when firm 1 makes all the investments, a symmetric proof holds for the other case.

The proof proceeds in the following way. We consider the pair of strategies where firm 2 never makes the investments, and firm 1 maximizes its profits acting as a monopolist constrained to charge the price equal to the initial value of the cost $c_{0}$. We then show that this indeed constitutes the pure strategy Markov perfect equilibrium of the Bertrand investment game by showing that neither firm has an incentive to deviate. For firm 2 the proof of the latter requires transfinite induction on the state of the art cost of production $c$. Recall that transfinite induction works as follows. We first show that a set of hypotheses ("the result") holds when the state of the art cost of production is $c=0$. The "inductive step" is to show that if these hypotheses hold for all $c^{\prime}<c$, then they hold for $c$ as well. Transfinite induction then guarantees that the result holds for all $c$, similar to the way the proof by induction would work if $c$ was discretized into a countable set of possible values.

Consider first firm 1 under the assumption that firm 2 follows the strategy of never investing. The fact that firm 1 will adopt a socially optimal investment strategy follows immediately from this assumption and Lemma 1. Since firm 1 knows that firm 2 will not invest, and the price of the good is fixed at $c_{0}$, firm 1 maximizes its profits by adopting an investment strategy that minimizes its present discounted costs of production and investment from any given starting node in the game $\left(c_{1}, c_{2}, c\right)$, in particular the apex, $\left(c_{0}, c_{0}, c_{0}\right)$. For some of the points, it may be optimal for firm 1 not to invest, however when this is the case, it would not be socially optimal for investment to occur there as well.

Consider next firm 2, assuming that firm 1 follows the socially optimal investment policy. For the described "monopoly" equilibrium to take place we need to prove that not investing is the optimal response of firm 2 along the equilibrium path. We do this by transfinite induction using inductive hypothesis that

$$
\begin{array}{r}
v_{N, 2}\left(c_{1}, c_{2}, c\right)=0 \text { and } \max \left(v_{N, 2}\left(c_{1}, c_{2}, c\right), v_{I, 2}\left(c_{1}, c_{2}, c\right)\right)=0 \\
\text { for all states }\left(c_{1}, c_{2}, c\right) \in S \text { where } c_{1} \leq c_{2} .
\end{array}
$$

It follows from Lemma A.3 that (47) holds in all states $\left(c_{1}, c_{2}, 0\right)$ where $c_{1} \leq c_{2}$. Namely, it holds in the single no investment equilibria at the states $(0,0,0)$ and $\left(0, c_{2}, 0\right), c_{2}>0$, per (33), and in 
the pure strategy eqilibria where firm 1 invests and firm 2 does not invest in the states $\left(c_{1}, c_{2}, 0\right)$, $c_{1}>0, c_{2}>0$. This establishes the base for the transfinite induction.

Now for the inductive step, we prove that if the hypothesis holds for $c^{\prime}<c$, then it also holds at the state of the art cost $c$, i.e. for all points $\left(c_{1}, c_{2}, c\right) \in S$ where $c_{1} \leq c_{2}$. Note that under the inductive hypothesis the Bellman equations for firm 2 simplify due to the fact that the function $h_{2}\left(c_{1}, c_{2}, c\right)$ in (46) is zero at these states. To prove the inductive hypothesis for $c^{\prime}=c$ we consider several subsets of the state points.

First, it is easy to show that in the point $(c, c, c)$ the value functions for firm 2 are $v_{N, 2}(c, c, c)=0$ and $v_{I, 2}(c, c, c)=-K(c)$, and thus the inductive hypothesis holds.

Second, consider the points $\left(c, c_{2}, c\right)$ where $c_{2}>c$. In these points firm 1 has already attained the state of the art production cost, and therefore has no incentive to invest. It is easy to show that in these points $v_{N, 1}\left(c, c_{2}, c\right)=v_{I, 1}\left(c, c_{2}, c\right)+K(c)$, and therefore $P_{1}\left(c, c_{2}, c\right)=0$. Then from (44) and (45) we have

$$
\begin{aligned}
v_{I, 2}\left(c, c_{2}, c\right) & =-K(c)+\beta \pi(c \mid c) \max \left(v_{N, 2}(c, c, c), v_{I, 2}(c, c, c)\right)=-K(c), \\
v_{N, 2}\left(c, c_{2}, c\right) & =\beta \pi(c \mid c) \max \left(v_{N, 2}\left(c, c_{2}, c\right),-K(c)\right) .
\end{aligned}
$$

We conclude that $v_{N, 2}\left(c, c_{2}, c\right)=0$, and therefore the inductive hypothesis holds.

Third, consider the points $\left(c_{1}, c_{2}, c\right) \in S$ where $c<c_{1} \leq c_{2}$ and assume $P_{1}\left(c_{1}, c_{2}, c\right)=1$. In this case the Bellman equations for firm 2 give

$$
\begin{aligned}
v_{I, 2}\left(c_{1}, c_{2}, c\right) & =-K(c)+\beta \pi(c \mid c) \max \left(v_{N, 2}(c, c, c), v_{I, 2}(c, c, c)\right)=-K(c), \\
v_{N, 2}\left(c_{1}, c_{2}, c\right) & =\beta \pi(c \mid c) \max \left(v_{N, 2}\left(c, c_{2}, c\right), v_{I, 2}\left(c, c_{2}, c\right)\right)=0 .
\end{aligned}
$$

Therefore the inductive hypothesis also holds when it is optimal for firm 1 to invest.

Finally, consider the points $\left(c_{1}, c_{2}, c\right) \in S$ where $c<c_{1} \leq c_{2}$ in the case when it is optimal for firm 1 not to invest, i.e. $P_{1}\left(c_{1}, c_{2}, c\right)=0$. This is the most complicated case because there is a potential for firm 2 to use the non-investment by firm 1 as an opportunity to sneak in and leapfrog firm 1 to become the new low cost leader. We now show that this "off the equilibrium path deviation" is not optimal for firm 2. While such a deviation will reduce the profits of firm 1 (by permanently lowering the price from $c_{0}$ to $c_{1}$ ), by the inductive hypothesis the behavior of the firms will not change in any subgame $\left(c_{1}, c_{2}, c^{\prime}\right)$ for $c^{\prime}<c$, so that firm 1 will resume playing the role of the monopolist investor and choose the timing of its subsequent investments in a socially optimal manner whereas firm 2 will not invest in any such subgame. The only effect of the deviation by firm 2 is to lower the revenues firm 1 can expect to get in any subgame following the deviation. However we now show that firm 2 will not find it optimal to deviate because it can only obtain temporary cost leadership for a random duration $\tilde{\tau}$ until the state of the art cost $c$ transits to some lower $\operatorname{cost} c_{\tilde{\tau}}<c$, at which point the game enters a subgame $\left(c_{1}, c, c_{\tilde{\tau}}\right)$ where firm 1 is the monopolist (and thus follows the socially optimal investment strategy) and firm 2 is the high cost follower and earns zero expected discounted profits, i.e. $V_{2}\left(c_{1}, c, c \tilde{\tau}\right)=0$ since the value for firm 2 is zero for all $c^{\prime}<c$, by our inductive hypothesis. In summary, under a deviation by firm 2 the game will spend $\tilde{\tau}$ periods in the state $\left(c_{1}, c, c\right)$, during which firm 2 wiil earn a per period profit of $c_{1}-c$. 
Thus, the total expected gain to firm 2 from deviating at $\left(c_{1}, c_{2}, c\right)$ is $\Delta-K(c)$ where $\Delta$ is given by

$$
\Delta=\beta E\left\{\sum_{t=1}^{\tilde{\tau}} \beta^{t-1}\left(c_{1}-c\right)\right\}=\frac{\left(c_{1}-c\right)\left(\beta-E\left\{\beta^{\tilde{\tau}}\right\}\right)}{1-\beta} .
$$

By Lemma A.1 $\Delta<K(c)$, and thus from the Bellman equations (44) that take the form

$$
\begin{aligned}
v_{I, 2}\left(c_{1}, c_{2}, c\right) & =\Delta-K(c)<0, \\
v_{N, 2}\left(c_{1}, c_{2}, c\right) & =\beta \pi(c \mid c) \max \left(v_{N, 2}\left(c_{1}, c_{2}, c\right), \Delta-K(c)\right),
\end{aligned}
$$

we conclude that $v_{N, 2}\left(c_{1}, c_{2}, c\right)=0$. Therefore the inductive hypothesis still holds.

We have shown that the inductive hypothesis (47) indeed holds in all points $\left(c_{1}, c_{2}, c\right) \in S$ where $c_{1} \leq c_{2}$ at the level of the state of the art cost $c$. Then by transfinite induction, it holds for all levels of $c$. This implies that the best response of firm 2 to the socially optimal investment strategy of firm 1 is not to invest when $c_{1} \leq c_{2}$. Because the "monopoly" equilibrium path is fully contained within this subset of the state space due to the fact that only firm 1 makes the cost reducing investments, this suffice to establish the "monopoly" equilibrium as a Markov perfect equilibrium of the whole Bertrand investment game. In this equilibrium firm 1 makes all the investments and follows the socially optimal investment policy, thus making it fully efficient.

Statement 3. (If Assumption 2 (Continuity), Assumption 3 (Threshold Monotonicity), and Assumption 4 (Value Monotonicity) hold, there exists a unique zero profit symmetric mixed strategy equilibrium (SZP-MSE) in the simultaneous move game that is "zero profit" in the sense that the expected payoffs to both firms in the subgames starting at all diagonal states $\left(c, c, c^{\prime}\right) \in S$ with $c^{\prime} \in[0, c]$. In addition, if the firms are in the mixed strategy region of the state space (i.e. where $c_{1} \geq \bar{c}(c)$ and $c_{2} \geq \bar{c}(c)$ where $\bar{c}(c)$ is the threshold defining the mixed strategy region), then the value function of the high cost firm is 0 and the value function of the low cost firm is strictly positive.)

Proof. Recall, that a mixed strategy equilibrium is an MPE of the overall game that involves playing mixed strategies at least in some states on the equilibrium path, i.e. the subset of points in the state space which are visited with positive probability under the equilibrium. We also distinguish the MPE for the full game from the MPE of its stage games - that is, the set of equilibria at any particular point in the state space $\left(c_{1}, c_{2}, c\right)$. It may happen that at some points in the support of a mixed strategy equilibrium that it is not socially optimal to invest, and thus the only stage game MPE is a pure strategy equilibrium where neither firm invests. However, we rule out the case when it is not socially optimal to invest at all in the sense of Assumption 1 at the initial point of the game. This implies that at least for some interior state $\left(c_{1}, c_{2}, c\right) \in S$ where $c_{1}<c$ and $c_{2}<c$, the firms invest with positive probability. As shown below this ensures the existence of a mixed strategy MPE for the full game.

The candidate symmetric mixed strategy equilibrium is the one where at all diagonal points we have $v_{1}\left(c, c, c^{\prime}\right)=v_{2}\left(c, c, c^{\prime}\right)=0$ for $c^{\prime} \leq c$ and in the mixed strategy region where $c_{1} \geq \bar{c}(c)$ and $c_{2} \geq \bar{c}(c)$ the firm with strictly lower cost (i.e. firm 1 in states $\left(c_{1}, c_{2}, c\right) \in S$ where $c_{2}>c_{1} \geq c$ ) 
has a positive value function and the firm with the high cost value function equal to zero. We also show that in this MPE the value functions and probability of investment are symmetric in their first two arguments, so this MPE is also a symmetric equilibrium. As we noted in the discussion of this Theorem in section 4.1, these results do not rule out the possibility that there also exist pure strategy regions of the state space where one firm invests and the other doesn't. As we discussed, it is even possible in cases where the cost of investment $K(c)$ is sufficiently low that there exist regions where the high cost firm invests and the low cost doesn't invest, and in such regions the high cost firm can have a strictly positive value function. The existence of such regions do not contradict the main result we prove in statement 3 of Theorem 2.

We prove the result by showing that the described equilibrium is indeed supported as the solution to the system of Bellman equations of the two firm (40) and (44). As for Statement 2 the proof is by transfinite induction using the following

Inductive Hypothesis for all $c^{\prime}<c$ the following properties hold:

1. The mixed strategy region is the orthant $\left\{\left(c_{1}, c_{2}, c^{\prime}\right) \mid c_{1} \in\left[\bar{c}\left(c^{\prime}\right), c_{0}\right], c_{2} \in\left[\bar{c}\left(c^{\prime}\right), c_{0}\right]\right\}$ defined by a threshold $\bar{c}\left(c^{\prime}\right)>c^{\prime}$ that by Assumption 3 is a continuous and non-decreasing function of $c^{\prime}$ for all $c^{\prime} \in[0, c)$. There are $3 \mathrm{MPE}$ at every point in this region, two pure anti-coordination MPE and a single SZP-MSE, which constitutes the selected MPE at each point in this region.

2. The value functions $\left(v_{1}, v_{2}\right)$ are symmetric in their $\left(c_{1}, c_{2}\right)$ arguments (i.e. $v_{1}\left(c_{1}, c_{2}, c^{\prime}\right)=$ $\left.v_{2}\left(c_{2}, c_{1}, c^{\prime}\right)\right)$ and are continuous and monotonic in the mixed strategy region of the state space. Specifically, by Assumption 4 this property holds at $c^{\prime}=0$ and by the transfinite inductive hypothesis $v_{1}\left(c_{1}, c_{2}, c^{\prime}\right)$ is non-increasing in $c_{2}$ and non-decreasing in $c_{1}$ for $c_{1}>$ $c_{2} \geq c^{\prime}$.

3. The value functions satisfy the zero profit and Bertrand properties in the mixed strategy region, specifically

$$
\begin{array}{r}
v_{1}\left(c_{1}, c_{2}, c^{\prime}\right)=0 \text { for all states }\left(c_{1}, c_{2}, c\right) \in S \text { where } c_{1} \geq c_{2} \geq \bar{c}\left(c^{\prime}\right), \\
v_{1}\left(c_{1}, c_{2}, c^{\prime}\right)>0 \text { for all states }\left(c_{1}, c_{2}, c^{\prime}\right) \in S \text { where } \bar{c}\left(c^{\prime}\right) \leq c_{1}<c_{2}, \\
\text { and symmetrically for firm } 2 .
\end{array}
$$

The strategy of the proof is to show the that inductive hypothesis holds in the endgame where $c=0$ (thus establishing the base case for the transfinite induction argument) and then show that if the Inductive Hypothesis (49) holds for all $c^{\prime}<c$ then the desired properties also hold at $c$. It then follows by the principle of transfinite induction that Theorem 2 is proved, i.e. the stated results hold for all $c \in\left[0, c_{0}\right]$.

First we prove the base case for transfinite induction, namely that the stated properties of the SZP-MSE hold in the endgame where $c=0$. We already established in Lemma A.3 that there is a threshold $\bar{c}(0)=K(0)(1-\beta) / \beta$ such that whenever $c_{1} \geq \bar{c}(0)$ and $c_{2} \geq \bar{c}(0)$ that there exists a unique mixed strategy MPE in any such state. It is easy to see that in the mixed strategy region the value functions for both firms are continuous functions of $\left(c_{1}, c_{2}\right)$ and by by Assumption 4 $v_{1}\left(c_{1}, c_{2}, 0\right)$ decreasing in $c_{1}$ and increasing in $c_{2}$. Thus items 1 and 2 of the Inductive Hypothesis hold in the base case where $c=0$. 
It remains to show that the zero profit and symmetry properties (49) hold in the base case. We have already established that the Inductive Hypothesis holds when $c=0$ and either $c_{1}=0$ or $c_{2}=0$ in the proof of Lemma A.3, see (32) and (33). Consider the interior states $\left(c_{1}, c_{2}, 0\right) \in S$ where $c_{1}>0$ and $c_{2}>0$. If it is socially optimal not to invest, i.e. $\min \left(c_{1}, c_{2}\right)<(1-\beta) K(0) / \beta$, firm 1 chooses not to invest and earns a positive profit $\frac{c_{1}-c_{2}}{1-\beta}$ in states $c_{1}<c_{2}$ and zero otherwise. If on the other hand, investment is socially optimal, by Lemma A.3 there is a mixed strategy equilibrium where firms are indifferent between investing and not investing, in particular $v_{N, 1}\left(c_{1}, c_{2}, 0\right)=v_{I, 1}\left(c_{1}, c_{2}, 0\right)$. Since current profit is positive for the cost leader, i.e. $r_{1}\left(c_{1}, c_{2}\right)>0$ for $c_{1}<c_{2}$ and zero otherwise, it follows from (34) that $v_{N, 1}\left(c_{1}, c_{2}, 0\right)=v_{I, 1}\left(c_{1}, c_{2}, 0\right)>0$ for $c_{1}<c_{2}$ and $v_{N, 1}\left(c_{1}, c_{2}, 0\right)=v_{I, 1}\left(c_{1}, c_{2}, 0\right)=0$ for $c_{1} \geq c_{2}$. A symmetric argument holds for firm 2 . Further, it is not difficult to show that symmetry in the payoffs and equilibrium strategies for the two firms holds for $c=0: v_{N, 1}\left(c_{1}, c_{2}, 0\right)=v_{N, 2}\left(c_{2}, c_{1}, 0\right)$ and $v_{I, 1}\left(c_{1}, c_{2}, 0\right)=v_{I, 2}\left(c_{2}, c_{1}, 0\right)$, and $P_{1}\left(c_{1}, c_{2}, 0\right)=P_{2}\left(c_{2}, c_{1}, 0\right)$. Thus, the Inductive Hypothesis (49) holds for $c=0$, which establishes the base case for the transfinite induction.

Now for the inductive step, where we prove if the Inductive Hypothesis (49) holds for all $c^{\prime}<c$, then it also holds at $c$.

First we show that $\bar{c}(c)$ exists and $\bar{c}(c) \geq \bar{c}\left(c^{\prime}\right)$ for $c^{\prime}<c$. This follows directly from the continuity and monotonicity properties of $\bar{c}(c)$ in item 1 of the Inductive Hypothesis. Define $\bar{c}(c)=\lim _{c^{\prime} \uparrow c} \bar{c}\left(c^{\prime}\right)$. This limit exists by the continuity of $\bar{c}\left(c^{\prime}\right)$ for $c^{\prime}<c$ by item 1 in the Inductive hypothesis. Further it must be the case that $\bar{c}(c) \geq \bar{c}\left(c^{\prime}\right)$ for $c^{\prime}<c$ since $\bar{c}\left(c^{\prime}\right)$ is a non-decreasing function of $c^{\prime}$ and thus its limit $\bar{c}(c)$ can be no smaller than $\bar{c}\left(c^{\prime}\right)$ for any $c^{\prime}<c$.

Below we will use the Inductive Hypothesis and the continuity of the value functions in the mixed strategy region to prove that the mixed strategy region when the state of the art is $c$ is given by the orthant $\left\{\left(c_{1}, c_{2}\right) \mid c_{1} \in\left[\bar{c}(c), c_{0}\right], c_{2} \in\left[\bar{c}(c), c_{0}\right]\right\}$. By the monotonicity property of the threshold function $\bar{c}(c)$ this mixed strategy orthant at $c$ will be a subset of the mixed strategy orthants for all $c^{\prime}<c$.

We now show that $\bar{c}(c)$ not only exists and is at least as large as $\bar{c}\left(c^{\prime}\right)$ for all $c^{\prime} \leq c$, but it is in fact the threshold defining the mixed strategy region at $c$. Consider the subset of diagonal states at $c:\left\{(\hat{c}, \hat{c}, c) \mid \hat{c} \in\left[c, c_{0}\right]\right\}$. We will proceed to show that if $\hat{c}<\bar{c}(c)$ then it is not optimal for either firm to invest given the belief that their opponent will not invest, and thus this subset of the diagonal states are in the no investment region (i.e. it is a subset of a Type 2 equilibrium region). We will show that in the no investment region we must have $v_{1}(\hat{c}, \hat{c}, c)=v_{2}(\hat{c}, \hat{c}, c)=0$. On the other hand if $\hat{c} \geq \bar{c}(c)$ we will show that it is optimal for the firms to invest (again conditional on the hypothesis that the opponent firm does not invest). Thus this subset of the diagonal states are in the mixed strategy region (i.e. is is a subset of the Type 1 equilibrium region). To prove this we first need to establish properties of the $h_{1}\left(c_{1}, c_{2}, c\right)$ and $h_{2}\left(c_{1}, c_{2}, c\right)$ functions defined in (42) and (46) above.

It is easy to see from (42) that the Inductive Hypothesis implies $h_{1}\left(c_{1}, c_{2}, c\right)=0$ when $c_{1} \geq c_{2}$, $h_{1}\left(c_{1}, c_{2}, c\right)>0$ when $c_{1}<c_{2}$, and analogously for $h_{2}\left(c_{1}, c_{2}, c\right)$ defined in (46). Specifically from the definition of $h_{1}\left(c_{1}, c_{2}, c\right)$ in equation (42), if $c_{1} \geq \bar{c}(c)$ and $c_{2} \geq \bar{c}(c)$, then it is the case that $c_{1} \geq$ $\bar{c}\left(c^{\prime}\right)$ and $c_{2} \geq \bar{c}\left(c^{\prime}\right)$ for all $c^{\prime}<c$. By the the zero profit property (49) it follows that $h_{1}\left(c_{1}, c_{2}, c\right)=0$ if $c_{1} \geq c_{2}$ and $h_{1}\left(c_{1}, c_{2}, c\right)>0$ if $c_{1}<c_{2}$ since the integrands $v_{1}$ and $v_{2}$ have these properties by 
the Inductive Hypothesis (49). Similar results hold for $h_{2}$ and by the symmetry property and the Inductive Hypothesis, so it follows that $h_{1}$ and $h_{2}$ are symmetric in the $\left(c_{1}, c_{2}\right)$ arguments, i.e. $h_{1}\left(c_{1}, c_{2}, c\right)=h_{2}\left(c_{2}, c_{1}, c\right)$ for all $c_{1} \geq c$ and $c_{2} \geq c$. It is also easy to see that because the mixed strategy regions are nested, and the Continuity Assumption 2 holds, then $h_{1}$ and $h_{2}$ inherit the same continuity and monotonicity properties (49) of the value functions that they are integrals of, as can be easily verified from formula (42).

To prove the remaining parts of the Inductive Hypothesis we consider several subsets of the state points. Consider first the corner state, $(c, c, c)$. Under the Inductive Hypothesis the Bellman equations (40) reduce to

$$
\begin{aligned}
v_{N, 1}(c, c, c) & =\beta \pi(c \mid c) \max \left[v_{N, 1}(c, c, c), v_{I, 1}(c, c, c)\right], \\
v_{I, 1}(c, c, c) & =-K(c)+\beta \pi(c \mid c) \max \left[v_{N, 1}(c, c, c), v_{I, 1}(c, c, c)\right],
\end{aligned}
$$

which implies that $v_{N, 1}(c, c, c)=0, v_{I, 1}(c, c, c)=-K(c)$ and $\max \left[v_{N, 1}(c, c, c), v_{I, 1}(c, c, c)\right]=0$. A symmetric argument holds for firm 2 .

Consider next the edge states $\left(c_{1}, c, c\right)$ where firm 2's cost equals the state of the art cost $c$, and firm 1 is the high cost follower, i.e. $c_{1}>c$. From the Bellman equations (44) it is apparent that $v_{N, 2}\left(c_{1}, c, c\right)=v_{I, 2}\left(c_{1}, c, c\right)+K(c)$ so firm 2 will not invest at these states as long as $K(c)>0$. Then, under the Inductive Hypothesis (49) it is easy to see that the Bellman equations (40) for firm 1 reduce to

$$
\begin{aligned}
v_{N, 1}\left(c_{1}, c, c\right) & =\beta \pi(c \mid c) \max \left[v_{N, 1}\left(c_{1}, c, c\right), v_{I, 1}\left(c_{1}, c, c\right)\right] \\
v_{I, 1}\left(c_{1}, c, c\right) & =-K(c)+\beta \pi(c \mid c) \max \left[v_{N, 1}(c, c, c), v_{I, 1}(c, c, c)\right] .
\end{aligned}
$$

With the above result for the point $(c, c, c)$, it follows that $v_{I, 1}\left(c_{1}, c, c\right)=-K(c), v_{N, 1}\left(c_{1}, c, c\right)=0$, and $\max \left[v_{N, 1}\left(c_{1}, c, c\right), v_{I, 1}\left(c_{1}, c, c\right)\right]=0$. Symmetric arguments hold for firm 2 at the edge states $\left(c, c_{2}, c\right), c_{2}>c$.

Consider next the edge states $\left(c, c_{2}, c\right), c_{2}>c$ where firm 1 is the low cost leader. It follows immediately from the Bellman equations (40) that $v_{N, 1}\left(c, c_{2}, c\right)=v_{I, 1}\left(c, c_{2}, c\right)+K(c)$. We just established above for firm 2 that $v_{N, 2}\left(c, c_{2}, c\right)=\max \left[v_{N, 2}\left(c, c_{2}, c\right), v_{I, 2}\left(c, c_{2}, c\right)\right]=0$, and thus $P_{2}\left(c, c_{2}, c\right)=0$. Together with the Inductive Hypothesis this leads to

$$
v_{N, 1}\left(c, c_{2}, c\right)=\frac{c_{2}-c+\beta h_{1}\left(c, c_{2}, c\right)}{1-\beta \pi(c \mid c)}=\max \left[v_{N, 1}\left(c, c_{2}, c\right), v_{I, 1}\left(c, c_{2}, c\right)\right]>0 .
$$

Symmetric arguments hold for firm 2 at the edge states $\left(c_{1}, c, c\right), c_{1}>c$.

Finally, consider the interior states $\left(c_{1}, c_{2}, c\right)$, where $c_{1}>c$ and $c_{2}>c$, and again start with firm 1. Combining the Inductive Hypothesis with the results above, it is easy to show that $H_{1}(c, c, c)=H_{1}\left(c_{1}, c, c\right)=0$, so the Bellman equations (40) can be further simplified to

$$
\begin{aligned}
& v_{N, 1}\left(c_{1}, c_{2}, c\right)=r_{1}\left(c_{1}, c_{2}\right) \\
& \quad+\beta\left(1-P_{2}\left(c_{1}, c_{2}, c\right)\right)\left(h_{1}\left(c_{1}, c_{2}, c\right)+\pi(c \mid c) \max \left[v_{N, 1}\left(c_{1}, c_{2}, c\right), v_{I, 1}\left(c_{1}, c_{2}, c\right)\right]\right), \\
& v_{I, 1}\left(c_{1}, c_{2}, c\right)=r_{1}\left(c_{1}, c_{2}\right)-K(c)+\beta\left(1-P_{2}\left(c_{1}, c_{2}, c\right)\right) H_{1}\left(c, c_{2}, c\right), \\
& H_{1}\left(c, c_{2}, c\right)=\frac{h_{1}\left(c, c_{2}, c\right)+\pi(c \mid c)\left(c_{2}-c\right)}{1-\beta \pi(c \mid c)} .
\end{aligned}
$$


First we establish that the key property of "zero values on the diagonals" holds, i.e. $v_{1}(\hat{c}, \hat{c}, c)=$ $v_{2}(\hat{c}, \hat{c}, c)=0$ for all $\hat{c} \in\left[c, c_{0}\right]$. Since $r_{1}(\hat{c}, \hat{c})=r_{2}(\hat{c}, \hat{c})=0$ at all diagonal states, it follows that the Bellman equation (53) above that the value of investing for firm 1 reduces to

$$
v_{1}(\hat{c}, \hat{c}, c)=-K(c)+\beta\left[1-P_{2}(\hat{c}, \hat{c}, c)\right] H_{1}(c, \hat{c}, c),
$$

and a similar equation holds for $v_{2}(\hat{c}, \hat{c}, c)$. If $K(c)>\beta H_{1}(c, \hat{c}, c)$ then it is easy to see that firm 1 would not want to invest in any circumstance at the point $(\hat{c}, \hat{c}, c)$, and by symmetry neither would firm 2, so this implies that this point lies in the Type 2 no investment region of the state space. We conclude that

$$
v_{1}(\hat{c}, \hat{c}, c)=v_{1, N}(\hat{c}, \hat{c}, c)>v_{I, 1}(\hat{c}, \hat{c}, c)
$$

and using the fact that $h_{1}(\hat{c}, \hat{c}, c)=0$ (already established above) and the top equation of (53) we conclude that $v_{1}(\hat{c}, \hat{c}, c)=0$ and by a symmetrical argument we conclude that $v_{2}(\hat{c}, \hat{c}, c)=0$ as well.

Note that by the Inductive Hypothesis, for all $c^{\prime}<c$ we have that $\bar{c}\left(c^{\prime}\right)$ is the threshold for the Type 1 mixed strategy region so that firm 1 is indifferent between investing and not investing when its cost is $c^{\prime}$ and its opponent's cost is $\bar{c}\left(c^{\prime}\right)$. This implies the following equation holds for each $c^{\prime}<c$

$$
K\left(c^{\prime}\right)\left[1-\beta \pi\left(c^{\prime} \mid c^{\prime}\right)\right]=\beta\left[h_{1}\left(c^{\prime}, \bar{c}\left(c^{\prime}\right), c^{\prime}\right)+\pi\left(c^{\prime} \mid c^{\prime}\right)\left(\bar{c}\left(c^{\prime}\right)-c^{\prime}\right)\right] .
$$

By Assumption 2 and the Inductive Hypothesis, both sides of equation (56) are continuous functions of $c^{\prime}$, and so by Assumption 3 in the limit as $c^{\prime} \rightarrow c$ we have that this indifference condition also holds at $c$

$$
K(c)[1-\beta \pi(c \mid c)]=\beta\left[h_{1}(c, \bar{c}(c), c)+\pi(c \mid c)\left(\bar{c}(c)-c^{\prime}\right)\right] .
$$

Further via the strict monotonicity of $v_{1}\left(c, c_{2}, c^{\prime}\right)$ in $c_{2}$ for $c^{\prime}<c$ by the Inductive Hypothesis, it follows that if $c_{2}<\bar{c}(c)$ then the right hand side of equation (57) is strictly less than the right hand side, and if $c_{2}>\bar{c}(c)$ the right hand side is strictly greater than the left hand side. This implies that $\bar{c}(c)$ is the threshold for the Type 2 mixed strategy region (at least along the diagonal $(\hat{c}, \hat{c}, c)$ ), and thus if $\hat{c} \geq \bar{c}(c)$ the point $(\hat{c}, \hat{c}, c)$ is in the Type 1 mixed strategy region. In the diagonal points in this region it is easy to see from equation (53) that a unique mixed strategy equilibrium will exist with investment probabilities given by

$$
P_{1}(\hat{c}, \hat{c}, c)=P_{2}(\hat{c}, \hat{c}, c)=1-\frac{K(c)[1-\beta \pi(c \mid c)]}{\beta\left[\pi(c \mid c)(\bar{c}(c)-c)+h_{1}(c, \bar{c}(c), c)\right]} .
$$

Further, since both firms must be indifferent between investing and not investing in a mixed strategy equilibrium, we must have for each $\hat{c} \geq c$

$$
\begin{aligned}
& v_{1}(\hat{c}, \hat{c}, c)=v_{1, N}(\hat{c}, \hat{c})=v_{1, I}(\hat{c}, \hat{c}, c) \\
& v_{2}(\hat{c}, \hat{c}, c)=v_{2, N}(\hat{c}, \hat{c})=v_{2, I}(\hat{c}, \hat{c}, c)
\end{aligned}
$$

and so by the first equation of (53) it follows that $v_{1}(\hat{c}, \hat{c}, c)=v_{2}(\hat{c}, \hat{c}, c)=0$. Thus, the key result of Theorem 2 holds for all $c \in\left[0, c_{0}\right]$ by transfinite induction. 
To complete the proof, it remains to show that for the off-diagonal points in the mixed strategy region that a) the continuity and monotonicity properties of the value function hold, and b) that the Bertrand Property holds, i.e. the value of the high cost firm is positive and the low cost firm is zero. We also need to verify that when the state of the art cost is $c$ the mixed strategy region is indeed the orthant defined by $\left\{\left(c_{1}, c_{2}\right) \mid c_{1} \geq \bar{c}(c), c_{2} \geq \bar{c}(c)\right\}$. Further, the equation defining the mixed strategy for the high cost firm is a quadratic equation that can have potentially two possible solutions. We will verify that one of the roots lies in the unit interval and hence for each $\left(c_{1}, c_{2}\right)$ in the mixed strategy region the SZP-MSE is unique.

Let $\Upsilon\{\cdot \leq \cdot\}$ denote an "indicator correspondence" which takes the values 0 and 1 similar to the usual indicator function, except that $\Upsilon$ takes all values in the unit interval $[0,1]$ when the inequality in the curly brackets is satisfied with equality. ${ }^{14}$ The best response of firm 1 to the investment choice probability $P_{2}=P_{2}\left(c_{1}, c_{2}, c\right)$ of firm 2 is then given by $\Upsilon\left\{v_{N, 1}\left(c_{1}, c_{2}, c, P_{2}\right) \leq\right.$ $\left.v_{I, 1}\left(c_{1}, c_{2}, c, P_{2}\right)\right\}$, where the last argument is added to emphasize the dependence on firm 2's investment probability $P_{2}$. All equilibria at the state $\left(c_{1}, c_{2}, c\right)$ are then the intersection points of $\Upsilon\left\{v_{N, 1}\left(c_{1}, c_{2}, c, P_{2}\right) \leq v_{I, 1}\left(c_{1}, c_{2}, c, P_{2}\right)\right\}$ and $\Upsilon\left\{v_{N, 2}\left(c_{1}, c_{2}, c, P_{1}\right) \leq v_{I, 2}\left(c_{1}, c_{2}, c, P_{1}\right)\right\}$ in the unit square on the plane $\left(P_{1}, P_{2}\right)$, with mixed strategy equilibria given by the intersection points on the interior of this square.

It follows from (53) that the best response correspondence of firm 1 can be expressed using a quadratic polynomial as

$$
P_{1}\left(c_{1}, c_{2}, c\right)=\Upsilon\left\{A_{1}+B_{1} P_{2}+C_{1} P_{2}^{2} \leq 0\right\},
$$

where the coefficients are given by

$$
\begin{aligned}
A_{1}= & \beta \pi(c \mid c) r_{1}\left(c_{1}, c_{2}\right)+\beta h_{1}\left(c_{1}, c_{2}, c\right) \\
& +(1-\beta \pi(c \mid c))\left[K(c)-\beta H_{1}\left(c, c_{2}, c\right)\right], \\
B_{1}= & -\beta h_{1}\left(c_{1}, c_{2}, c\right)-\beta \pi(c \mid c)\left[r_{1}\left(c_{1}, c_{2}\right)-K(c)+\beta H_{1}\left(c, c_{2}, c\right)\right] \\
& +\beta(1-\beta \pi(c \mid c)) H_{1}\left(c, c_{2}, c\right), \\
C_{1}= & \beta^{2} \pi(c \mid c) H_{1}\left(c, c_{2}, c\right) .
\end{aligned}
$$

with analogous expressions holding for firm 2 .

Consider first the states $\left(c_{1}, c_{2}, c\right)$ where $c_{1} \leq c_{2}$, i.e. firm 1 is the cost leader and firm 2 is the cost follower. For the latter we have $h_{2}\left(c_{1}, c_{2}, c\right)=r_{2}\left(c_{1}, c_{2}\right)=0$, and the polynomial coefficients (61) for firm 2 simplify to

$$
\begin{aligned}
& A_{2}=(1-\beta \pi(c \mid c))\left[K(c)-\beta H_{2}\left(c_{1}, c, c\right)\right], \\
& B_{2}=\beta \pi(c \mid c) K(c)+\beta(1-2 \beta \pi(c \mid c)) H_{2}\left(c_{1}, c, c\right), \\
& C_{2}=\beta^{2} \pi(c \mid c) H_{2}\left(c_{1}, c, c\right) .
\end{aligned}
$$

It is not hard to verify that the roots of the corresponding polynomial are

$$
P_{1}^{*}=1-\frac{K(c)}{\beta H_{2}\left(c_{1}, c, c\right)} \leq 1 \quad \text { and } \quad P_{1}^{* *}=1-\frac{1}{\beta \pi(c \mid c)} \leq 0
$$

\footnotetext{
${ }^{14}$ Here again the proof follows closely the proof of Theorem 6 in Iskhakov, Rust, Schjerning (2016).
} 
where the $P_{1}^{* *}$ clearly does not fall in the unit interval. Depending on the sign of $P_{1}^{*}$, firm 2 either does not invest for any level of $P_{1}$ (if $P_{1}^{*}<0$ ) or invests with probability one when $P_{1} \leq P_{1}^{*}$ if $P_{1}^{*}$ is positive.

Denote the relevant threshold for the investment cost to distinguish between these two cases as

$$
K_{1}\left(c_{1}, c_{2}, c\right)=\beta H_{2}\left(c_{1}, c, c\right)=\beta \frac{h_{2}\left(c_{1}, c, c\right)+\pi(c \mid c)\left(c_{1}-c\right)}{1-\beta \pi(c \mid c)},
$$

where the second equality is due to (53). We conclude that the depending on the size of $K(c)$ firm 2's (the high cost follower) best response to firm 1 is given by one of the following two cases

$$
\begin{aligned}
& \text { Case (1) } K(c) \geq K_{1}\left(c_{1}, c_{2}, c\right) \Rightarrow P_{2}\left(c_{1}, c_{2}, c, P_{1}\right)=0 \text { for all } P_{1}, \\
& \text { Case (2) } K(c)<K_{1}\left(c_{1}, c_{2}, c\right) \Rightarrow P_{2}\left(c_{1}, c_{2}, c, P_{1}\right)= \begin{cases}1, & \text { if } 0 \leq P_{1}<P_{1}^{*}, \\
{[0,1],} & \text { if } P_{1}=P_{1}^{*}, \\
0, & \text { if } P_{1}^{*}<P_{1} \leq 1 .\end{cases}
\end{aligned}
$$

Consider now the best response of firm 1 in states $\left(c_{1}, c_{2}, c\right), c_{1} \leq c_{2}$ when it is the low cost leader. The polynomial coefficients (61) in this case do not simplify in any significant way, as $h_{1}\left(c_{1}, c_{2}, c\right)>0$ and $r_{1}\left(c_{1}, c_{2}\right)=c_{2}-c_{1}>0$. Consequently, the roots of the polynomial in the best response correspondence are rather involved. We can, however, calculate the values of the polynomial at the ends of the unit interval to get some insights into where the roots may be located. We have

$$
\begin{aligned}
& A_{1}+B_{1} P_{2}+\left.C_{1} P_{2}^{2}\right|_{P_{2}=1}=K(c) \\
& A_{1}+B_{1} P_{2}+\left.C_{1} P_{2}^{2}\right|_{P_{2}=0}=K(c)(1-\beta \pi(c \mid c))-\beta\left(h_{1}\left(c, c_{2}, c\right)-h_{1}\left(c_{1}, c_{2}, c\right)+\pi(c \mid c)\left(c_{1}-c\right)\right)
\end{aligned}
$$

As long as $K(c)>0$, the first line in (66) implies that the best response correspondence of firm 1 is zero at $P_{2}=1$ when firm 2 invests with certainty. But from the second line it follows that for $P_{2}<1$ the best response of firm 1 depends on the cost of investment $K(c)$. Namely, if

$$
K(c) \leq K_{2}\left(c_{1}, c_{2}, c\right)=\beta \frac{h_{1}\left(c, c_{2}, c\right)-h_{1}\left(c_{1}, c_{2}, c\right)+\pi(c \mid c)\left(c_{1}-c\right)}{1-\beta \pi(c \mid c)}
$$

the polynomial is negative at $P_{2}=0$, ensuring that a single root lies in the unit interval. In the opposite case, additional arguments are needed to differentiate between the case when both roots lie in the unit interval and the case when the polynomial has no real roots, but as it will become obvious below, both of these cases lead to the same set of equilibria. We conclude that the depending on the size of $K(c)$ firm 1 (cost leader's) best response to the investment probability of firm 2 is given by one of the following two cases

$$
\begin{aligned}
& \text { Case (1) } K(c) \geq\left. K_{2}\left(c_{1}, c_{2}, c\right) \Rightarrow P_{1}\left(c_{1}, c_{2}, c, P_{2}\right)\right|_{P_{2}=0}=0, \\
& \text { Case (2) } K(c)<K_{2}\left(c_{1}, c_{2}, c\right) \Rightarrow P_{1}\left(c_{1}, c_{2}, c, P_{2}\right)= \begin{cases}1, & \text { if } 0 \leq P_{2}<P_{2}^{*}, \\
{[0,1],} & \text { if } P_{2}=P_{2}^{*}, \\
0, & \text { if } P_{2}^{*}<P_{2} \leq 1,\end{cases}
\end{aligned}
$$


where $P_{2}^{*}$ denotes the single root of the polynomial in the unit interval in the corresponding case.

It follows that all possible stage game equilibria at points $\left(c_{1}, c_{2}, c\right), c_{1} \leq c_{2}$ are given by the combinations of cases in (65) with (68). The following configurations are possible:

Type $1 K(c) \leq K_{1}\left(c_{1}, c_{2}, c\right)$ and $K(c) \leq K_{2}\left(c_{1}, c_{2}, c\right)$ : similar to the interior points of the bottom layer game (when $c=0$ ), there are three stage equilibria, two pure strategy anti-coordination equilibria, and one mixed strategy equilibrium,

Type $2 K_{1}\left(c_{1}, c_{2}, c\right)<K(c)$ and $K_{2}\left(c_{1}, c_{2}, c\right)<K(c)$ : there is one pure strategy equilibrium in which neither firm invests,

Type $3 K_{1}\left(c_{1}, c_{2}, c\right)<K(c) \leq K_{2}\left(c_{1}, c_{2}, c\right)$ : there is one pure strategy equilibrium in which firm 1 (cost leader) invests with probability one, and firm 2 (cost follower) does not invest.

Type $4 K_{2}\left(c_{1}, c_{2}, c\right)<K(c) \leq K_{1}\left(c_{1}, c_{2}, c\right)$ : there is one pure strategy equilibrium in which firm 2 (cost follower) invests with probability one, and firm 1 (cost leader) does not invest.

Note that Statement 3 of Theorem 2 only applies to points in the Type 1 mixed strategy region for $c_{1}<c_{2}$, i.e. the off-diagonal points. First we prove that when the state of the art cost is $c$ that the Type 1 mixed strategy region is indeed the orthant of $\left(c_{1}, c_{2}\right)$ points to the northeast of $(\bar{c}(c), \bar{c}(c))$. Consider the high cost firm 2. Using the Bellman equation for firm 2 (44) and the properties of the $h_{2}$ functions, it is easy to show via simple algebra that the condition for firm 2 to want to invest given that it believes that firm 1 will not invest (which is a necessary condition for a mixed strategy equilibrium to exist) is equivalent to the following inequality

$$
K(c)[1-\beta \pi(c \mid c)] \leq \beta\left[\pi(c \mid c)\left(c_{1}-c\right)+h_{2}\left(c_{1}, c, c\right)\right]=\beta\left[\pi(c \mid c)\left(c_{1}-c\right)+\beta h_{1}\left(c, c_{1}, c\right)\right],
$$

where we used the symmetry property of the value functions (and therefore of $h_{1}$ and $h_{2}$ that hold by the Inductive Hypothesis. However by the monotonicity and continuity properties of $h_{1}$ (again due to the Inductive Hypothesis), it follows that inequality (69) holds if and only if $c_{1} \geq \bar{c}(c)$. Now consider firm 1, the low cost firm. Using the Bellman equation for firm 1 in (40) to derive a condition that firm 1 would want to invest given that it believes that firm 2 will not invest results in the following inequality

$$
v_{I, 1}\left(c_{1}, c_{2}, c\right) \geq \frac{c_{2}-c_{1}+\beta h_{1}\left(c_{1}, c_{2}, c\right)}{1-\beta \pi(c \mid c)} .
$$

However the Bellman equation (40) and the properties of the $h_{1}$ function imply the following formula for $V_{I, 1}\left(c_{1}, c_{2}, c\right)$

$$
v_{I, 1}\left(c_{1}, c_{2}, c\right)=-K(c)+\frac{c_{2}-c_{1}+\beta \pi(c \mid c)\left(c_{1}-c\right)+\beta h_{1}\left(c, c_{2}, c\right)}{1-\beta \pi(c \mid c)} .
$$

Inserting the formula for $v_{I, 1}$ in equation (71) into inequality (70) and simplifying, we obtain the following inequality which must hold in order to guarantee that firm 1 would want to invest at the state $\left(c_{1}, c_{2}, c\right)$ given that it believes its opponent will not invest

$$
K(c)[1-\beta \pi(c \mid c)] \leq \beta \pi(c \mid c)\left(c_{1}-c\right)+\beta\left[h_{1}\left(c, c_{2}, c\right)-h_{1}\left(c_{1}, c_{2}, c\right)\right] .
$$


Assume that $c_{1} \geq \bar{c}(c)$ and $c_{2} \geq \bar{c}(c)$. Then by Assumption 3 and the Inductive Hypothesis, it follows that $c_{1} \geq \bar{c}\left(c^{\prime}\right)$ and $c_{2} \geq \bar{c}\left(c^{\prime}\right)$ and thus $\left(c_{1}, c_{2}, c^{\prime}\right)$ is in the Type 1 mixed strategy for all $c^{\prime}<c$. This implies that the following inequality must hold for all $c^{\prime}<c$

$$
K\left(c^{\prime}\right)\left[1-\beta \pi\left(c^{\prime} \mid c^{\prime}\right)\right] \leq \beta \pi\left(c^{\prime} \mid c^{\prime}\right)\left(c_{1}-c^{\prime}\right)+\beta\left[h_{1}\left(c^{\prime}, c_{2}, c^{\prime}\right)-h_{1}\left(c_{1}, c_{2}, c^{\prime}\right)\right] .
$$

By Assumption 2 and the Inductive Hypothesis, all functions on the left and right hand sides of inequality (73) are continuous functions of $c^{\prime}$ and thus in the limit as $c^{\prime} \uparrow c$ it follows that inequality (72) must also hold. Thus we conclude that if $c_{1} \geq \bar{c}(c)$ and $c_{2} \geq \bar{c}(c)$ then the point $\left(c_{1}, c_{2}, c\right)$ is in the Type 1 mixed strategy region, thereby verifying via transfinite induction that the Type 1 mixed strategy region is defined by a continuous and non-decreasing threshold function $\bar{c}(c)$ for all $c \in\left[0, c_{0}\right]$.

To complete the proof we need to verify that the value functions $\left(v_{1}, v_{2}\right)$ have the properties of a) symmetry, b) continuity and c) monotonicity for all points $\left(c_{1}, c_{2}, c\right)$ in the Type 1 mixed strategy region, and also satisfy the Bertrand property, i.e. the value of the high cost firm is zero and the low cost firm is strictly positive. For any $\left(c_{1}, c_{2}, c\right) \in S$ satisfying $c_{1} \geq \bar{c}(c)$ and $c_{2} \geq$ $\bar{c}(c)$, Assumption 3 implies that $\left(c_{1}, c_{2}, c^{\prime}\right)$ is in the mixed strategy region for all $c^{\prime} \leq c$ and hence $v_{1}\left(c_{1}, c_{2}, c^{\prime}\right)$ and $v_{2}\left(c_{1}, c_{2}, c^{\prime}\right)$ satisfy the symmetry, continuity and monotonicity properties by the Inductive Hypothesis. Taking limits we have

$$
\begin{aligned}
& v_{1}\left(c_{1}, c_{2}, c\right)=\lim _{c^{\prime} \uparrow c} v_{1}\left(c_{1}, c_{2}, c^{\prime}\right) \\
& v_{2}\left(c_{1}, c_{2}, c\right)=\lim _{c^{\prime} \uparrow c} v_{2}\left(c_{1}, c_{2}, c^{\prime}\right)
\end{aligned}
$$

so $v_{1}\left(c_{1}, c_{2}, c\right)$ and $v_{2}\left(c_{1}, c_{2}, c\right)$ must inherit the symmetry, continuity and monotonicity properties as limits. In particular, if $c_{1}<c_{2}$, if follows from the second limit in (74) that $v_{2}\left(c_{1}, c_{2}, c^{\prime}\right)=0$ and thus $v_{2}\left(c_{1}, c_{2}, c\right)=0$. Similarly since $v_{1}\left(c_{1}, c_{2}, c^{\prime}\right)>0$ it follows that $v_{1}\left(c_{1}, c_{2}, c\right) \geq 0$. However the following formula for $v_{1}\left(c_{1}, c_{2}, c\right)$ holds in the mixed strategy region

$$
v_{1}\left(c_{1}, c_{2}, c\right)=\frac{c_{2}-c_{1}+\beta\left[1-P_{2}\left(c_{1}, c_{2}, c\right)\right] h_{1}\left(c_{1}, c_{2}, c\right)}{1-\beta \pi(c \mid c)\left[1-P_{2}\left(c_{1}, c_{2}, c\right)\right]}
$$

which implies that $v_{1}\left(c_{1}, c_{2}, c\right)$ is actually strictly positive.

Having established the existence of the SZP-MSE, we now prove its uniqueness. This result also follows from transfinite induction, but it holds essentially by the recursive way the SZPMSE is constructed. First consider the endgame, $c=0$ which constitutes the base case for the proof of uniqueness by transfinite induction. By Lemma A.3 there are only Type 1 (3 possible MPE) and Type 2 (one pure strategy no investment MPE) regions. Obviously the no investment equilibrium in the Type 2 no investment region is unique, and in the Type 1 mixed strategy region, Lemma A. 3 proved that there there are only 3 possible MPE in that region: two pure strategy "anticoordination" equilibria and a single mixed strategy equilibrium. By construction, the SZP-MSE amounts to an equilibrium selection rule that picks the unique mixed strategy MPE in every point of the Type 1 mixed strategy region, so the SZP-MSE is unique in all end game states.

By the Inductive Hypothesis and the proof above, at every point $\left(c_{1}, c_{2}, c^{\prime}\right) \in S$ for all state of the art costs $c^{\prime}<c$, there are only 4 possible equilibrium regions. In the Type 2,3 , or 4 regions 
there is only one possible pure strategy MPE so uniqueness at these points is automatic. In the Type 1 mixed strategy region, there are only 3 possible MPE only one of which is the selected SZP-MSE, so uniqueness of the SZP-MSE holds for all $c^{\prime}<c$ by the Inductive Hypothesis.

We have already shown above that by Assumption 2 there are also only 4 possible equilibrium regions at $c$, and in the mixed strategy region we also pick the unique SZP-MSE that exists at every point in the Type 1 mixed strategy orthant. It follows from transfinite induction that the SZP-MSE is unique for all points $\left(c_{1}, c_{2}, c\right) \in S$.

Statement 4. (If Assumption 2 (Continuity) holds, the convex hull of the set of the expected discounted equilibrium payoffs to the two firms in all MPE equilibria of simultaneous move game at the apex $\left(c_{0}, c_{0}, c_{0}\right)$ is a triangle with vertices $(0,0),\left(0, V_{M}\right)$ and $\left(V_{M}, 0\right)$, where $V_{M}=V_{i}\left(c_{0}, c_{0}, c_{0}\right)$ is the expected discounted payoff of firm $i$ which makes all investments in the monopoly equilibrium.).

Proof. Statement 2 of this Theorem ensures the existence of two monopoly equilibria in the simultaneous move game, proving that the two corner payoff points $\left(V_{M}, 0\right)$ and $\left(0, V_{M}\right)$ exist, where $V_{M}=v_{N, 1}\left(c_{0}, c_{0}, c_{0}\right)=v_{N, 2}\left(c_{0}, c_{0}, c_{0}\right)$ is the monopoly payoff at the initial node (apex) $\left(c_{0}, c_{0}, c_{0}\right) \in S$. Since the monopoly profit equals the full social surplus and is efficient, it is infeasible to obtain any payoff higher than the line segment joining these two monopoly payoff points,and thus all payoffs for all equilibria in the simultaneous move game (which are generally less than $100 \%$ efficient) must lie below the line segment joining the two monopoly payoff points. Finally, Statement 3 of this Theorem guarantees the existence of the zero payoff point at the origin $(0,0)$. Obviously the convex hull of these three payoff points equals the full triangle, and thus any point in this triangle can be an expected payoff to the two firms if we allow stochastic equilibrium selection rules (i.e. selecting one of these three possible "extremal equilibria" with probabilities $\left(p_{1}, p_{2}, p_{3}\right)$ with $p_{1}+p_{2}+p_{3}=1$ and $\left.p_{i} \geq 0, i \in\{1,2,3\}\right)$.

Corollary 2.1 (Independence property of the mixed strategy equilibrium)

Proof. If $c_{1} \geq \bar{c}(c)$ and $c_{2} \geq \bar{c}(c)$ so we are in the Type 1 mixed strategy region, then if $c_{2}>$ $c_{1}$ firm 2 is the high cost follower and so by Theorem 2 we have $v_{2}\left(c_{1}, c_{2}, c\right)=0$. Using the Bellman equation for firm 2, (44) and setting $v_{I, 2}\left(c_{1}, c_{2}, c\right)=0$ and solving for $P_{1}\left(c_{1}, c_{2}, c\right)$ we obtain formula (18) which obviously does not depend on $c_{2}$. By symmetry the result also holds for firm 2 when $c_{2}<c_{1}$.

Corollary 2.2 (Sufficient condition to rule out Type 4 regions)

Proof. We need to show that in any state $\left(c_{1}, c_{2}, c\right)$ where the high cost firm finds it optimal to invest under the belief that the low cost firm will not invest, then it must also hold that the low cost firm will also want to invest if it believes that the high cost firm will not invest. Suppose that $c_{1}<c_{2}$ so firm 2 is the high cost firm. In the proof of Theorem 2 we derived an inequality (69) for firm 2 to want to invest given that it believes firm 1 will not invest. Similarly, inequality (72) is the 
necessary and sufficient condition for firm 1 to want to invest given that it believes firm 2 will not invest. Notice that using the fact that $h_{1}\left(c_{1}, c_{1}, c\right)=0$ we can rewrite inequality (69) as follows

$$
K(c)[1-\beta \pi(c \mid c)] \leq \beta\left[\pi(c \mid c)\left(c_{1}-c\right)+\beta\left[h_{1}\left(c, c_{1}, c\right)-h_{1}\left(c_{1}, c_{1}, c\right)\right]\right] .
$$

Thus, it is sufficient to show that the following inequality holds

$$
h_{1}\left(c, c_{2}, c\right)-h_{1}\left(c_{1}, c_{2}, c\right) \geq h_{1}\left(c, c_{1}, c\right)-h_{1}\left(c_{1}, c_{1}, c\right) .
$$

However because the $h_{1}$ function is simply an integral of the values of $v_{1}$ at lower state of the art cost values as given in equation (42), the result follows if it holds that

$$
v_{1}\left(c, c_{2}, c^{\prime}\right)-v_{1}\left(c_{1}, c_{2}, c^{\prime}\right) \geq v_{1}\left(c, c_{1}, c^{\prime}\right)-h_{1}\left(c_{1}, c_{1}, c^{\prime}\right),
$$

for all $c^{\prime}<c$. However this is precisely the increasing differences property (21) that is assumed to hold as part of the hypothesis for Corollary 2.2.

Below we prove Theorem 3 by induction, and the following Lemma provides a key result we need to establish the base case for the inductive argument.

Lemma A.6 (Equilibrium of the alternating move end game). Consider the end game, i.e. where $c=0$, of the alternating move version of the Bertrand pricing and investment game. Consider without loss of generality a situation where it is firm 1's turn to move (i.e. only firm 1 is allowed to invest). We have

$$
P_{1}\left(c_{1}, c_{2}, 0,1\right)= \begin{cases}1 & \text { if } \beta \min \left(c_{1}, c_{2}\right) /(1-\beta) \geq K(0) \\ 0 & \text { if } \beta \min \left(c_{1}, c_{2}\right) /(1-\beta)<K(0) .\end{cases}
$$

The payoff to firm 1 for $c_{1} \leq c_{2}$ is given by

$$
v_{1}\left(c_{1}, c_{2}, 0,1\right)= \begin{cases}c_{2}-c_{1} & \text { if } \beta \min \left(c_{1}, c_{2}\right) /(1-\beta) \leq K(0) \\ c_{2}-c_{1}-K(0)+\beta c_{2} /(1-\beta) & \text { if } \beta \min \left(c_{1}, c_{2}\right) /(1-\beta)>K(0),\end{cases}
$$

and for $c_{1}>c_{2}$ we have

$$
v_{1}\left(c_{1}, c_{2}, 0,1\right)= \begin{cases}0 & \text { if } \beta \min \left(c_{1}, c_{2}\right) /(1-\beta) \leq K(0) \\ -K(0)+\beta c_{2} /(1-\beta) & \text { if } \beta \min \left(c_{1}, c_{2}\right) /(1-\beta)>K(0) .\end{cases}
$$

For firm 2, which cannot invest, its payoff when it is socially optimal to invest is

$$
v_{2}\left(c_{1}, c_{2}, 0,1\right)= \begin{cases}0 & \text { if } c_{1}<c_{2} \\ c_{1}-c_{2} & \text { if } c_{1} \geq c_{2}\end{cases}
$$

and its payoff when it is not optimal to invest is given by

$$
v_{2}\left(c_{1}, c_{2}, 0,1\right)= \begin{cases}0 & \text { if } c_{1}<c_{2} \\ \left(c_{1}-c_{2}\right) /(1-\beta) & \text { if } c_{1} \geq c_{2} .\end{cases}
$$

Symmetric results hold when it is firm 2's right to move in the end game. 
Proof. Suppose that is it socially optimal to invest, so that $\beta \min \left(c_{1}, c_{2}\right) /(1-\beta) \geq K(0)$. If it is firm 1's turn to invest and it chooses to invest, its payoff is $c_{2}-c_{1}-K(0)+\beta c_{2} /(1-\beta) \geq 0$ if $c_{2} \geq c_{1}$ and $-K(0)+\beta c_{2} /(1-\beta) \geq 0$ if $c_{2}<c_{1}$, so it is only necessary to show these payoffs are higher than the payoffs to firm 1 from not investing in both of these respective cases. It is not hard to show that if firm 1 decides not to invest its expected payoff will be less than it can get from investing. The best possible case for firm 1 is when $f(1 \mid 1)=1$, i.e. it always has the the right to invest. In this case the payoff to not investing is $\left(c_{2}-c_{1}\right) /(1-\beta)$ when $c_{2}>c_{1}$ and 0 when $c_{2} \leq c_{1}$. In either case it is easy to see that the condition that investment is socially optimal, $\beta \min \left(c_{1}, c_{2}\right) /(1-\beta) \geq K(0)$ implies that that the payoff to firm 1 from investing is higher than the payoff from not investing, so it follows that $P_{1}\left(c_{1}, c_{2}, 0,1\right)=1$ as claimed in equation (79). If $f(1 \mid 1)<1$ the gain in firm 1's payoffs to investing immediately only increases, since firm 1 loses the chance to move in the next period with certainty, and it is easy to show that the inequality for the social optimality of investment implies that firm 2 will want to invest whenever it gets the turn to invest. Once firm 2 invests firm 1 and leapfrogs firm 1, then firm 1's payoffs fall to zero, even when $c_{1}<c_{2}$. This reduces the payoff to firm 1 from choosing not to invest compared to the best case scenario where $f(1 \mid 1)=1$.

If it is not socially optimal to invest, $\beta \min \left(c_{1}, c_{2}\right) /(1-\beta)<K(0)$, then it is easy to show that both firms obtain a lower payoff from investing compared to not investing. For example if $c_{1}<c_{2}$, then firm 1 is the low cost firm, and the inequality that investment is not socially optimal implies that the payoff to firm 1 from investing is less than the payoff from not investing is $c_{2}-c_{1}-K(0)+\beta c_{2} /(1-\beta)<\left(c_{2}-c_{1}\right) /(1-\beta)$. Similarly, for firm 2 , the high cost firm, the payoff to not investing is 0 but the payoff to investing is $-K(0)+\beta c_{1} /(1-\beta)<0$, due to the assumption that investment is not socially optimal. This establishes that $P_{1}\left(c_{1}, c_{2}, 0,1\right)=0$ when investment is not socially optimal, thereby verifying the second part of equation (79).

The equations for the payoffs to firm 1, (80) and (81), immediately follow from the equilibrium investment probability in (79) since firm 1 will invest immediately if it is socially optimal to invest and then it obtains the payoffs in the second part of equations (80) and (81) and if investment is not socially optimal, firm 1 does not invest and it obtains the payoffs in the first part of equations (80) and (81). The payoff to firm 2 given in equation (82) then follows immediately from the fact that firm 1 invests and thus leapfrogs firm 2 when it is socially optimal to invest, thus preempting firm 2 from being able to invest. Thus firm 2 will earn either $c_{1}-c_{2}$ if $c_{2}<c_{1}$ or 0 if $c_{1} \leq c_{2}$ as given in in equation (82). However if it is not socially optimal to to invest, neither firm invests so firm 2 either gets a payoff of 0 if $c_{1}<c_{2}$, or $\left(c_{1}-c_{2}\right) /(1-\beta)$ if $c_{1} \geq c_{2}$, as shown in equation (83).

Theorem 3 (The set of equilibrium payoffs in the Alternating Move Game)

Proof. By Theorem 1, if investment is optimal for the social planner, then inequality (29) cannot hold. By Lemma A.2, it follows that no investment cannot be an MPE outcome in the alternating move game similar to the simultaneous move game. We have established in Lemma A.6 above that if investment is socially optimal, then there is a unique pure strategy MPE in the end game in which the firm whose turn it is to invest will receive strictly positive expected profits in equilibrium. This establishes the base case for a proof by induction that whichever firm has the turn to invest will have a strictly positive expected payoff in not just the end game but in all states in the game. So 
our inductive hypothesis is that this property holds at all state of the art costs $c^{\prime}<c$. That is, and without loss of generality, the inductive hypothesis is that $v_{1}\left(c_{1}, c_{2}, c^{\prime}, 1\right)>0$ when firm 1 is the firm whose turn it is to invest. We now wish to show that this property must also hold at $c$. From the Bellman equation for firm 1 in the alternating move game, (7), we can immediately see that if $f(1 \mid 1)>0$ then due to the inductive hypotheis that $v_{1}\left(c_{1}, c_{2}, c^{\prime}, 1\right)>0$, it follows that the value of the decision not to invest is $v_{N, 1}\left(c_{1}, c_{2}, c, 1\right)>0$. Since $v_{1}\left(c_{1}, c_{2}, c, 1\right)$ is the maximum of the value of not investing and the value of investing, it follows that $v_{1}\left(c_{1}, c_{2}, c, 1\right)>0$. If $f(1 \mid 1)=0$ then $f(2 \mid 1)=1$ and this is a deterministically alternating move game. Then the value of not investing is given by the second integral expression in the first equation of the Bellman equation for firm 1 in (7). This term involves the values to firm 1 when it is not its turn to invest, but depends on whether firm 2 invests or does not invest, respectively, i.e. $v_{I, 1}\left(c_{1}, c_{2}, c^{\prime}, 2\right)$ and $v_{N, 1}\left(c_{1}, c_{2}, c^{\prime}, 2\right)$ given in the third and fourth equations of the Bellman equations for firm 1 in (7). Each of these equations, in turn, are a sum of three terms, the second of which is $f(1 \mid 2)$ (the probability that firm 1 will get to invest next period given that it is firm 2's turn to invest this period) times an integral of $v_{1}\left(c_{1}, c_{2}, c^{\prime}, 1\right)$. By the inductive hypothesis this integral must be strictly positive, and since it must be the case that $f(1 \mid 2)>0$ (otherwise the game would not be an alternating move game), it follows that both $v_{N, 1}\left(c_{1}, c_{2}, c, 2\right)$ and $v_{I, 1}\left(c_{1}, c_{2}, c, 2\right)$ are strictly positive. It then follows from the first equation of (7) that $v_{N, 1}\left(c_{1}, c_{2}, c, 1\right)$ is strictly positive, and thus that $v_{1}\left(c_{1}, c_{2}, c, 1\right)=$ $\max \left[v_{N, 1}\left(c_{1}, c_{2}, c, 1\right), v_{I, 1}\left(c_{1}, c_{2}, c, 1\right)\right]$ is strictly positive.

We conclude that $v_{1}\left(c_{1}, c_{2}, c, 1\right)$ is strictly positive for all $c \in\left[0, c_{0}\right]$. This means that at the apex or starting point of the game, we have $v_{1}\left(c_{0}, c_{0}, c_{0}, 1\right)>0$. A symmetric argument establishes that $v_{2}\left(c_{0}, c_{0}, c_{0}, 2\right)>0$. Thus, regardless of which firm has the right to move at the starting state of the game, $\left(c_{0}, c_{0}, c_{0}\right)$, at least one of the firm's values must be strictly positive.

From this it immediately follows that the set of all MPE payoffs at the starting point of the game cannot include the origin, $(0,0)$, whereas the origin was an element of the set of all MPE payoffs at the start of the game by Theorem 2. It follows that the convex hull of the set of MPE payoffs in the alternating move version of the leapfrogging game is a strict subset of the convex hull of the set of all MPE payoffs in the simultaneous move game as claimed.

Note that in our proof of Theorem 3 we did not attempt to prove that the two monopoly MPE (i.e. MPE where only one of the firm always invests, and at the socially optimal times, and the other firm never invests) are not supportable as MPE in the alternating move leapfrogging game even though we did prove that they are supportable as MPE in the simultaneous move version of the leapfrogging game in Theorem 2. Though this result does generally hold (for example, see panel (a) of Figure 6), it may be possible to construct counterexamples where the monopoly payoffs are possible in an alternating move leapfrogging game. For example, it may be possible to construct an example with deterministically improving technology and deterministically alternating moves where there are 3 socially optimal investments occurring at times 1, 3 and 5. If firm 1 moves at odd time periods and it is possible to show that firm 2 (which moves at even periods) would not want to invest at times $t=2$ and $t=4$, this would constitute an example where the monopoly MPE could in fact be achieved in the alternating move game. However we believe this would be a very special counterexample and the outcome we nearly always see from computations of the set of all MPE 
using the RLS algorithm is that the monopoly payoffs are not supportable in the alternating move game, so the convex hull of the set of all MPE payoffs is in strictly in the interior of the payoff triangle and does not include any of its three vertices.

We note that Theorem 6 implies that a zero payoff for both firms is approached in the limit as $\Delta t \rightarrow 0$ when $\pi\left(c_{t} \mid c_{t}\right)=0$ and the order of moves alternates deterministically. However in that case, since the equilibrium is unique, it follows that the monopoly payoff vertices are not supportable in the limit as $\Delta t \rightarrow 0$. Thus, even in limiting cases, the set of equilibrium payoffs in the alternating move game will be a strict subset of the payoff triangle that excludes the origin (zero expected profit mixed strategy payoff) and the two monopoly payoffs.

Theorem 4 (Sufficient conditions for uniqueness).

The proof requires some intermediary results.

Lemma A.7 (Efficiency of the alternating move end game). In the alternating move $(m \neq 0)$ end game $(c=0)$ in every state $\left(c_{1}, c_{2}, 0\right)$ there is a unique efficient equilibrium, i.e. both firms invest when it is their turn to invest if and only if investment would be optimal from the point of view of the social planner.

Proof. Consider the case where $c_{1}<c_{2}$. The proof for the case $c_{1} \geq c_{2}$ is symmetric to the one provided below for $c_{1}<c_{2}$ and is omitted for brevity. Suppose that it is socially optimal to undertake investment, i.e. $\beta c_{1} /(1-\beta)-K(0)>0$. We now show that in the unique equilibrium to the alternating move end game, both firms 1 and 2 would want to invest when it is their turn to invest, where uniqueness of equilibrium is a consequence of the uniqueness of the firms' best responses, and the fact that only one of the firm moves at a time. Consider firm 2's decision in this unique equilibrium. If firm 2 chooses to invest, its payoff is $v_{I, 2}\left(c_{1}, c_{2}, 0,2\right)=\beta c_{1} /(1-\beta)-K(0)$ and if it chooses not to invest its payoff is $v_{N, 2}\left(c_{1}, c_{2}, 0,2\right)=0$ since it believes that firm 1 will invest at its turn with probability 1 , which we will verify is true below. Thus, firm 2 will invest in equilibrium if and only if $\beta c_{1} /(1-\beta)-K(0)>0$, which is the same condition for optimal investment by the social planner.

Now consider firm 1. At it's turn to move the payoff to investing is

$$
v_{I, 1}\left(c_{1}, c_{2}, 0,1\right)=c_{2}-c_{1}+\beta c_{2} /(1-\beta)-K(0) .
$$

Since $c_{2}>c_{1}$ and by assumption $\beta c_{1} /(1-\beta)-K(0)>0$, it is easy to see that the payoff to investing is strictly positive for firm 1 . However we must also show that this is higher than the payoff it would get from not investing. Since firm 1 knows that firm 2 will invest when it gets a chance to move, the value to firm 1 to not investing is given by

$$
v_{N, 1}\left(c_{1}, c_{2}, 0,1\right)=c_{2}-c_{1}+\beta f(1 \mid 1)\left[c_{2}-c_{1}+\frac{\beta}{1-\beta} c_{2}-K(0)\right]+\beta f(2 \mid 1)\left[c_{2}-c_{1}\right] .
$$

If the posited equilibrium holds (i.e. it is optimal for firm 1 to invest), then we must have $v_{I, 1}\left(c_{1}, c_{2}, 0,1\right)>v_{N, 1}\left(c_{1}, c_{2}, 0,1\right)$, and using the formulas for these values given above, this is equivalent to

$$
\frac{\beta}{1-\beta} c_{2}-K(0)>\frac{\beta\left(c_{2}-c_{1}\right)}{1-\beta f(1 \mid 1)} .
$$


Notice that the right hand side of inequality (86) above is maximized when $f(1 \mid 1)=1$ (i.e. when it is always firm 1's turn to invest) and in this case this inequality is equivalent to $\beta c_{1} /(1-\beta)-$ $K(0)>0$, confirming that for all $f(1 \mid 1) \in[0,1]$ it is strictly optimal for firm 1 to invest when it is its turn to invest when it is socially optimal for this investment to occur.

Now consider the converse situation where it is not socially optimal to invest, and $\beta c_{1} /(1-$ $\beta)-K(0)<0$. Following the same reasoning as above, it is easy to see that it is not optimal for firm 2 to invest when it is its turn to invest since firm 2's payoff to investing is $v_{I, 2}\left(c_{1}, c_{2}, 0,2\right)=$ $\beta c_{1} /(1-\beta)-K(0)<0$ and its payoff to not investing is $v_{N, 2}\left(c_{1}, c_{2}, 0,2\right)=0$. Now we must show that firm 1, knowing that firm 2 will not want to invest at its turn, will also not want to invest when it is its turn. If firm 1 never invests, its payoff is

$$
v_{N, 1}\left(c_{1}, c_{2}, 0,1\right)=\frac{c_{2}-c_{1}}{1-\beta},
$$

and if it invests, its payoff is given by the same formula for $v_{I, 1}\left(c_{1}, c_{2}, 0,1\right)$ as given in equation (84) above. So the condition for investment not to be optimal for firm 1 is $v_{N, 1}\left(c_{1}, c_{2}, 0,1\right)>$ $v_{I, 1}\left(c_{1}, c_{2}, 0,1\right)$ which is algebraically equivalent to $\beta c_{1} /(1-\beta)-K(0)<0$, the condition for when it is not socially optimal for investment to occur.

Now we have the key result needed to prove Theorem 4.

Proof. When $\pi(c \mid c)=0$, the probability of remaining in any given state $\left(c_{1}, c_{2}, c\right) \in S$ is also zero. Using the Bellman equations (7) defining the firms' value functions for investing and not investing when it is their turn to invest, it is not difficult to see that each firm's values are independent of the probability that their opponent will invest in this case. That is, for firm 1 we have $v_{N, 1}\left(c_{1}, c_{2}, c, 1\right)$ and $v_{I, 1}\left(c_{1}, c_{2}, c\right)$ are independent of $P_{2}\left(c_{1}, c_{2}, c, 2\right)$, the probability that firm 2 will invest when it is its turn to invest. This implies that the probability that firm 1 will invest, $P_{1}\left(c_{1}, c_{2}, c, 1\right)$, is also independent of $P_{2}\left(c_{1}, c_{2}, c, 2\right)$, as it is given by formula (5) of section 2, which shows that $P_{1}\left(c_{1}, c_{2}, c, 1\right)$ is a logistic function of $v_{N, 1}\left(c_{1}, c_{2}, c, 1\right)$ and $v_{I, 1}\left(c_{1}, c_{2}, c, 1\right)$, both of which are independent of $P_{2}\left(c_{1}, c_{2}, c, 2\right)$. Similar arguments hold for firm 2, so that $P_{2}\left(c_{1}, c_{2}, c, 2\right)$ is independent of $P_{1}\left(c_{1}, c_{2}, c, 1\right)$. Since the value functions $\left(v_{N, 1}, v_{I, 1}, v_{N, 2}, v_{I, 2}\right)$ can be calculated recursively using the Bellman equations (5), and since Lemma A.7 establishes that there is always a unique (efficient) equilibrium in the end game states $\left(c_{1}, c_{2}, c\right)$, it follows that at every state $\left(c_{1}, c_{2}, c\right) \in S$ there is a unique stage game equilibrium with probabilities of investing given by $\left(P_{1}\left(c_{1}, c_{2}, c, 1\right), P_{2}\left(c_{1}, c_{2}, c, 2\right)\right)$, which depend on the value functions $\left(v_{N, 1}, v_{I, 1}, v_{N, 2}, v_{I, 2}\right)$ that are defined recursively via the Bellman equations (7). 